\title{
Epidemiological studies of schizophrenia
}

Citation for published version (APA):

Allardyce, J. G. S. (2009). Epidemiological studies of schizophrenia. [Doctoral Thesis, Maastricht University]. Maastricht University. https://doi.org/10.26481/dis.20090528ja

Document status and date:

Published: 01/01/2009

DOI:

10.26481/dis.20090528ja

Document Version:

Publisher's PDF, also known as Version of record

\section{Please check the document version of this publication:}

- A submitted manuscript is the version of the article upon submission and before peer-review. There can be important differences between the submitted version and the official published version of record.

People interested in the research are advised to contact the author for the final version of the publication, or visit the DOI to the publisher's website.

- The final author version and the galley proof are versions of the publication after peer review.

- The final published version features the final layout of the paper including the volume, issue and page numbers.

Link to publication

\footnotetext{
General rights rights.

- You may freely distribute the URL identifying the publication in the public portal. please follow below link for the End User Agreement:

www.umlib.nl/taverne-license

Take down policy

If you believe that this document breaches copyright please contact us at:

repository@maastrichtuniversity.nl

providing details and we will investigate your claim.
}

Copyright and moral rights for the publications made accessible in the public portal are retained by the authors and/or other copyright owners and it is a condition of accessing publications that users recognise and abide by the legal requirements associated with these

- Users may download and print one copy of any publication from the public portal for the purpose of private study or research.

- You may not further distribute the material or use it for any profit-making activity or commercial gain

If the publication is distributed under the terms of Article $25 \mathrm{fa}$ of the Dutch Copyright Act, indicated by the "Taverne" license above, 


\section{EPIDEMIOLOGICAL STUDIES OF SCHIZOPHRENIA}





\title{
EPIDEMIOLOGICAL STUDIES OF SCHIZOPHRENIA
}

\author{
PROEFSCHRIFT
}

ter verkrijging van de graad van doctor aan de Universiteit Maastricht, op gezag van de Rector Magnificus, Prof. mr. G.P.M.F. Mols,

volgens het besluit van het College van Decanen,

in het openbaar te verdedigen op

donderdag 28 mei 2009 om 14:00 uur

door

Judith Grace Shankland Allardyce 
PROMOTOR

Prof. dr. J. van Os

BEOORDELINGSCOMMISSIE

Prof. dr. M. de Vries (voorzitter)

Prof. dr. P. Jones, Department of Psychiatry, Cambridge University , UK

Prof. dr. R.M. Murray, Institute of Psychiatry, Kings College, London, UK

Prof.dr. H.M. van Praag

Prof. dr. G. van Widdershoven 


\section{IF (RUDYARD KIPLING 1865-1936)}

If you can keep your head when all about you Are losing theirs and blaming it on you;

If you can trust yourself when all men doubt you, But make allowance for their doubting too; If you can wait and not be tired by waiting, Or being lied about, don't deal in lies, Or being hated, don't give way to hating, And yet don't look too good, nor talk too wise: If you can dream -- and not make dreams your master; If you can think -- and not make thoughts your aim; If you can meet with Triumph and Disaster And treat those two imposters just the same; If you can bear to hear the truth you've spoken Twisted by knaves to make a trap for fools, Or watch the things you gave your life to, broken, And stoop and build 'em up with worn-out tools; If you can make one heap of all your winnings And risk it on one turn of pitch-and-toss, And lose, and start again at your beginnings And never breathe a word about your loss; If you can force your heart and nerve and sinew To serve your turn long after they are gone, And so hold on when there is nothing in you Except the Will which says to them: "Hold on!" If you can talk with crowds and keep your virtue, Or walk with kings -- nor lose the common touch, If neither foes nor loving friends can hurt you, If all men count with you, but none too much; If you can fill the unforgiving minute With sixty seconds' worth of distance run -Yours is the Earth and everything that's in it, And -- which is more -- you'll be a Man, my son! 



\section{Contents}

\section{Chapter 1}

Introduction

\section{Chapter 2}

Variation in the incidence of schizophrenia over time

A study examining trends over time (1979-1998) in the service based incidence rates for schizophrenia in Dumfries \& Galloway in South West Scotland

\section{Chapter 3}

Variation in the incidence of schizophrenia by place

A study comparing the service based incidence rates of schizophrenia in rural Dumfries \& Galloway in South West Scotland with urban Camberwell in South East London.

\section{Chapter 4}

Variation in the incidence of psychosis by area level social characteristics

A national record Linkage study investigating the association of first admission rates for psychosis and the areas based measures of social fragmentation, material deprivation and urban/rural index.

\section{Chapter 5}

Variation in the rates of psychosis by area level social characteristics. A systematic review.

\section{Chapter 6}

Variation in the incidence of schizophrenia by person

Extracts from a chapter in the next addition of the textbook Kaplan \& Saddock reviewing the current literature on known risk factors for the onset of schizophrenia.

\section{Chapter 7}

Defining caseness: Schizophrenia a provisional diagnostic category?

This paper reviews the diagnostic validity of the schizophrenia construct and suggests potential methods of improving its clinical usefulness.

\section{Chapter 8}

Discusses the conceptual background for including a dimensional component in the diagnosis of psychosis in future classification systems. 


\section{Chapter 9}

Diagnostic utility: of the diagnostic category schizophrenia

This study generates symptom dimensions using detailed psychopathological information from service based incidence cases of psychosis from Dumfries \& Galloway and compares their usefulness in discriminating between known risk factors for psychosis.

\section{Chapter 10}

Diagnostic utility: of the diagnostic category schizophrenia

This study examines the usefulness of symptom dimensions and DSM -IV diagnostic categories in predicting outcome at five years.

Chapter 11

Epilogue

Samenvatting (Summary in Dutch)

Acknowledgements

Curriculum Vitae 
CHAPTER 1

\section{Introduction}




\section{INTRODUCTION}

Schizophrenia is a devastating and often debilitating mental illness characterized by symptoms such as hallucinations, delusional believes, disorganized thinking, loss of goal directed behaviors and deterioration in social functioning. For some, the disease can be relatively mild with the person suffering one or several episodes with little or no lasting impairment. However, for approximately $47 \%$ of patients, schizophrenia is a disorder for life; with persistent symptoms or recurrent episodes associated with significant social disability. (Hegarty et al. 1994). It is an expensive illness to manage, recent estimates of total health service costs, in the United Kingdom (based on figures from 2004-2005) are in the order of $£ 2$ billion, making it the third most costly diagnostic group in Britain after (learning disability and stroke disorder) this represents 3\% of the total health care expenditure. The economic burden however extends well beyond the health care system to other care organizations and public sector bodies, such as housing departments and the criminal justice system. Further, 'hidden costs' include lost income due to employment difficulties, typically one fifth of all persons with schizophrenia are unable to find paid work, resulting in huge losses to the national economy. There is also a significant burden on family (and friends) e.g. lost leisure time, and employment opportunities. That is, schizophrenia has significant health, social and economic impacts on patients, their families and the wider society (Hegarty et al. 1994;Knapp M et al. 2004;Mangslore R and Knapp M 2007)."Aetiological elusiveness" Few mental disorders have been investigated with such persistence over decades with such patchy results as schizophrenia. Some advances have been made and a number of genetic and environmental factors have been identified (discussed in this thesis) which increase the risk of schizophrenia however they are generally of small/modest effect and are neither necessary or sufficient causal components. Using epidemiology to understand the cause of diseases. Epidemiology is a population science. The central paradigm of classical epidemiology is that systematic analyses of the patterns of disease will provide understandings to their causes and eventual control. The key strategy compares and contrasts aggregated disease pattern in populations over TIME, between PLACE and in different (subgroups of people defined by variation in demographic and risk factor exposures) PERSONS. Consequently, classical epidemiological studies measure the likelihood or probability of disease at the group level that is, what happens on average in a population. A person's risk may deviate from the estimated average. For example, the causal link for cigarette smoking and lung cancer is robust; however, an individual person's risk may differ from the estimated relative risk (for smokers) in the population, due to specific environmental or genetic factors, which reduce their susceptibility to the carcinogenetic effects of tobacco (Bhopal R. 2002). Variation in disease over time That diseases wax and wane in their population frequency as the result of medical /public health practice or natural causes is one of the axioms of classical epidemiology. Variation in disease occurs over time in populations because the characteristics of the people or their environment changes. Even if the social and physical environment were constant some patterns of disease over time would likely occur (of course much more slowly, over evolutionary time scales) for genetic changes are inevitable and will influence the onset and persistence of disease. Genetic changes arise from a number of processes including genetic drift, genetic mutation and epigenetic transmutations, which cause random variation in the gene frequency from generation to generation. In small populations, genetic drift can lead to important genetically driven disease differences however, in large populations genetic DNA (deoxyribonucleic acid) sequencing remains relatively stable over time. Classical epidemiological methodology assumes that changes in disease frequency within large 
populations occurring over short time period (few decades) are almost wholly due to environmental factors. Since the 1980's there have been a substantial number of studies suggesting that the first admission rates of schizophrenia have declined by as much as $50 \%$ in the latter part of the twentieth century. (Al Mousawi and Dunstan 1998;Brewin et al. 1997;de Alarcon and Seagroatt 1990;Der et al. 1990;Eagles and Whalley 1985;Eagles et al. 1988;Munk-Jorgensen 1986;Munk-Jorgensen and Jorgensen 1986;Munk-Jorgensen and Mortensen 1993;MunkJorgensen et al. 1992;Oldehinkel and Giel 1995;Osby et al. 2001;Suvisaari et al. 1999). While others studies have found significant increase in service defined incidence rates over the same period.(Castle et al. 1991;Preti and Miotto 2000;Boydell et al. 2003). Variation of disease rates by place Similar insights into aetiology can be determined by systematically examining variation in the rates of disease between populations with different demographic and environmental characteristics. Until recently, it was a generally held view that the incidence of schizophrenia was uniform. Indeed the WHO Collaborative study (Sartorius N. et al. 1996) which specifically set out to ascertain incidence rates for schizophrenia in rural and urban areas, in developed and developing countries, concluded that "schizophrenic illness, occur with comparable frequency in different populations". While this study was carefully executed and measured contemporaneously incidence in a number of different geographical areas using the same ascertainment methods, semi structured interview (PSE, present state examination) for diagnoses it may have been hampered by low power when comparing the narrowly defined schizophrenia. Since the publication of the WHO study a raft of studies have demonstrated spatial variation in the incidence of schizophrenia (McGrath et al. 2004). Real variation in time and place or artifact? For causal understandings of observed variation in rates of disease, the first step must be to exclude artifact because if the variation is an illusion then there is no epidemiological association to explain! (Bhopal R. 2002).

Variation in the rates of disease by time and place: Artefactual explanations

1. CHANCE- random fluctuation

2. DIFFERENTIAL ERROR OF OBSERVATION: sampling and information bias

3. VARIATION IN THE UNDERLYING SIZE \& STRUCTURE OF THE POPULATION

4. VARIATION IN HEALTH SEEKING BEHAVIOUR: this varies with level of knowledge, expectations, accessibility and acceptability of health care services.

5. DIAGNOSTIC ACCURACY: variation in and quality diagnostic facilities, level of skill and training

6. VARIATION IN DIAGNOSTIC CLASSIFICATION SYSTEMS \& FASHION: variation in clinical approaches to classification systems employed

7. VARIATION IN CASE ASCERTIANMENT: Variation in service administration and information systems.

8. VARIATION IN STATISTICAL ANALYSES: Different underlying population structures or changes in the standard populations used in adjusting disease rates for differences in age and sex.

Variation in person (clinical epidemiology: subgroup analyses) harnesses the inherent heterogeneity seen in populations. For example in a society where everyone smoked heavily, (uniform exposure) the classical epidemiological strategy of comparing and contrasting exposure would be impossible (Schunemann H J and Guyatt G H 2005) However examining the variation in susceptibility, onset, course and outcome of disease and the reasons behind this variation helps us to better understand the disease. through the systematic identification and evaluation 
of inherited and acquired characteristics of individuals', attributes, behaviors and exposures, known as risk factors (determinants). These risk factors increase an individual's likelihood of developing i) a precursor of the clinical disorder ( a condition or state preceding the recognized onset of a disease) ii) a clinical disease or iii) a particular course and outcome trajectory for the disease process. Determinants rarely act in isolation to increase risk; rather their effects are cumulative. Such complementary actions can be additive (the linear sum of the individual effects) or synergistic (interaction of effects) when individual risk factor effects are modified by particular environmental conditions, biological stochastic events or with other known risk factors. Further, there is no reason to assume that the risk factors for the emergence of a clinical disorder and those influencing persistence are congruent. Evidence that the same risk factor is implicated in both onset and persistence of a disease (discrete major effect) could aid our understanding of aetiological mechanisms. However, lack of, discrete effects suggests the course of a disease is not pre determined and is potentially modifiable after onset. Clinical epidemiology uses this variation in risk factor profiles to generate hypotheses and develop empirically based conceptual frameworks (models) of disease. Identifying the risk factors operating prior to the onset of disease are fundamental to effective evidence based primary prevention, while the risk factors predicting course and outcome help identify patients at higher risk of poor outcome, so guiding the development of secondary and tertiary preventative strategies and interventions tailored for specific individual's in clinical settings. Heterogeneity is the hallmark of schizophrenia, with manifest variation in risk factors for onset, symptomatology, course and outcome. Caseness: schizophrenia a provisional diagnostic category! Central to any epidemiological study is the issue of caseness. How we define the disease outcome determines the estimated rates in descriptive epidemiological studies and the calculated effect sizes in analytical studies. From the 1960's psychiatrists have expended much energy in improving the reliability of psychiatric diagnoses in general and schizophrenia in particular. This has resulted in a number of operational definitions of schizophrenia, which have accepted diagnostic reliability, but all vary and it is questionable which one should be adopted. The basic problem is that we do not know whether the concept of schizophrenia has any validity and even if it has, which operational definition best reflects that validity. (Andreasen 1995;Carlos E.Berganza et al. 2005;John S.Strauss 2005;Kendell R.E. 1989; Kendler 1980;Kessler 2002;Liddle 1987). That schizophrenia is at best a provisional diagnostic category has serious implications for the epidemiological study design and interpretation of the results. It handicaps our research efforts and impedes our aetiological understandings resulting in calls for the investigation of further investigation into its validity. 


\section{STATEMENT OF OBJECTIVES}

The aim of the current thesis is threefold.

\section{Part 1}

Describes studies investigates the variation in incidence of psychosis (more particularly schizophrenia) in time and place.

\section{Part 2}

Reviews the current literature on the variation in risk for schizophrenia by person (studies which examine the association of demographic and differential exposure (risk gradients) to putative risk factors with rates of schizophrenia).

\section{Part 3}

Reviews the validity of schizophrenia as a diagnostic construct and examines its diagnostic utility.

\section{REFERENCE LIST}

Al Mousawi,A.H. and Dunstan,F.D., 1998. Changes in the risk of schizophrenia in Scotland: is there an environmental factor? Schizophr Bull. 24, 529-535.

Andreasen,N.C., 1995. The validation of psychiatric diagnosis: new models and approaches. American Journal of Psychiatry 152(2):161-2.

Bhopal R., 2002. Cause and effect : The epidemiological approach. Concepts of epidemiology: an integrated introduction to the ideas, theories, principles and methods in epidemiology. Oxford University Press, New York.

Boydell,J., van Os,J., Lambri,M., Castle,D., Allardyce,J., McCreadie,R.G., and Murray,R.M., 2003. Incidence of schizophrenia in south-east London between 1965 and 1997. British Journal of Psychiatry 182:45-9.

Brewin,J., Cantwell,R., Dalkin,T., Fox,R., Medley,I., Glazebrook,C., Kwiecinski,R., and Harrison,G., 1997. Incidence of schizophrenia in Nottingham. A comparison of two cohorts, 1978-80 and 1992-94. Br J Psychiatry. 171, 140-144.

Carlos E.Berganza, Juan E.Mezzich, and Claire,P., 2005. Concepts of Disease: Their Relevance for Psychiatric Diagnosis and Classification. Psychopathology. 38, 166.

Castle,D., Wessely,S., Der,G., and Murray,R.M., 1991. The incidence of operationally defined schizophrenia in Camberwell, 1965-84. Br J Psychiatry. 159, 790-794.

de Alarcon,J.G. and Seagroatt,V., 1990. Trends in schizophrenia. Lancet. 335, 852-853.

Der,G., Gupta,S., and Murray,R.M., 1990. Is schizophrenia disappearing? Lancet. 335, 513-516.

Eagles,J.M., Hunter,D., and McCance,C., 1988. Decline in the diagnosis of schizophrenia among first contacts with psychiatric services in north-east Scotland, 1969-1984. Br J Psychiatry. 152, 793-798.

Eagles,J.M. and Whalley,L.J., 1985. Decline in the diagnosis of schizophrenia among first admissions to Scottish mental hospitals from 1969-78. Br J Psychiatry. 146, 151-154.

Hegarty,J.D., Baldessarini,R.J., Tohen,M., Waternaux,C., and Oepen,G., 1994. One hundred years of schizophrenia: a meta-analysis of the outcome literature.[see comment]. American Journal of Psychiatry. 151, 1409-1416.

John S.Strauss, 2005. Diagnosis and Reality: A Noun Is a Terrible Thing to Waste. Psychopathology. 38, 189.

Kendell R.E., 1989. Clinical validity. 471. Psychological Medicine. 45-55. 
Kendler,K.S., 1980. The nosologic validity of paranoia (simple delusional disorder). A review. Archives of General Psychiatry 37(6):699-706.

Kessler,R.C., 2002. Epidemiological perspectives for the Development of Future diagnostic systems. Psychopathology. $35,158-161$.

Knapp M, Mangslore R, and Simon J, 2004. Global costs of schizophrenia. Schizophrenia Bulletin. 30, 279-293.

Liddle,P.F., 1987. "Are there more than two syndromes in schizophrenia? A critique of the positiveegative dichotomy". British Journal of Psychiatry. 161, England.

Mangslore R and Knapp M, 2007. Cost of schizophrenia in England. Journal of Mental Health Policy and Economics. 10, 23-41.

McGrath,J., Saha,S., Welham,J., El Saadi,O., MacCauley,C., and Chant,D., 2004. A systematic review of the incidence of schizophrenia: the distribution of rates and the influence of sex, urbanicity, migrant status and methodology. BMC Medicine. 2, 13 .

Munk-Jorgensen,P., 1986. Decreasing first-admission rates of schizophrenia among males in Denmark from 1970 to 1984. Changing diagnostic patterns? Acta Psychiatr Scand. 73, 645-650.

Munk-Jorgensen,P. and Jorgensen,P., 1986. Decreasing rates of first-admission diagnoses of schizophrenia among females in Denmark 1970-84. Acta Psychiatr Scand. 74, 379-383.

Munk-Jorgensen,P., Lutzhoft,J.H., Jensen,J., and Stromgren,E., 1992. Trends in psychiatric hospitalization in Denmark: a 10-year register-based investigation. Acta Psychiatr Scand. 86, 79-83.

Munk-Jorgensen,P. and Mortensen,P.B., 1993. Is schizophrenia really on the decrease? Eur Arch Psychiatry Clin Neurosci. 242, 244-247.

Oldehinkel,A.J. and Giel,R., 1995. Time trends in the care-based incidence of schizophrenia. Br J Psychiatry. 167, 777782.

Osby,U., Hammar,N., Brandt,L., Wicks,S., Thinsz,Z., Ekbom,A., and Sparen,P., 2001. Time trends in first admissions for schizophrenia and paranoid psychosis in Stockholm County, Sweden. Schizophr Res. 47, 247-254.

Preti,A. and Miotto,P., 2000. Increase in first admissions for schizophrenia and other major psychoses in Italy. Psychiatry Res. 94, 139-152.

Sartorius N., Jablensky,A., Korten,A., and et al, 1996. Early manifestation and first contact rates of schizophrenia in different cultures. Psychological Medicine. 16, 909-928.

Schunemann H J and Guyatt G H, 2005. Clinical Epidemiology. Ahrens W and Pigeot I (Eds.), Handbook of Epidemiology. Springer, Berlin.

Suvisaari,J.M., Haukka,J.K., Tanskanen,A.J., and Lonnqvist,J.K., 1999. Decline in the incidence of schizophrenia in Finnish cohorts born from 1954 to 1965 . Arch Gen Psychiatry. 56, 733-740. 
Part 1 


\section{CHAPTER 2}

\section{Variation in the incidence of schizophrenia over time}

Allardyce, J., Morrison, G., van Os, J Kelly, J., Murray, R.M., and McCreadie, R.G. 2000 Schizophrenia is not disappearing in south-west Scotland.

British Journal Psychiatry 177:38-41. 


\section{Schizophrenia is not disappearing in south-west}

\section{Scotland}

\author{
J. ALLARDYCE, G. MORRISON, J. VAN OS, J. KELLY, R. M. MURRAY \\ and R. G. McCREADIE
}

\begin{abstract}
Background Recent work has reported a decline in the incidence of schizophrenia, but it is unclear if these findings reflect a true decrease in its incidence or are an artefact arising from methodological difficulties.
\end{abstract}

\begin{abstract}
Aims To take account of these methodological difficulties and report service-based incidence rates for schizophrenia in Dumfries and Galloway in south-west Scotland for 1979-98.
\end{abstract}

\section{Method Using both clinical diagnoses and diagnoses generated from the Operational Checklist for Psychotic Disorders (OPCRIT) computer algorithm for ICD-10 and DSM-IV schizophrenia, we measured change in the incidence rates over time. We used indirect \\ standardisation techniques and Poisson models to measure the rate ratio linear trend.}

Results There was a monotonic and statistically significant decline in clinically diagnosed schizophrenia. The summary rate ratio linear trend was 0.77 . However, using OPCRIT-generated ICD-10 and DSM-IV diagnoses, there was no significant difference over time.

\section{Conclusions OPCRIT-generated}

consistent diagnoses revealed no significant fall in the incidence of schizophrenia. Changes in diagnostic practice have caused the declining rates of clinically diagnosed schizophrenia in Dumfries and Galloway.

Declaration of interest This work was supported by the Stanley Foundation.
Dramatic declines (up to as much as $50 \%$ ) in the first admission rates for schizophrenia have been demonstrated in different countries and continents over the past few decades (Munk-Jørgensen, 1986; Joyce, 1987; Der et al, 1990; Suvisaari et al, 1999). Several of these reports have come from Scotland (Eagles \& Walley, 1985; Geddes et al, 1993). It is still not clear if this replicated finding reflects a true decrease in the incidence of schizophrenia or is simply an artefact (Jablensky, 1997). The uncertainty is due to the considerable methodological difficulties inherent in measuring trends over time in the incidence of schizophrenia. Because it is a rare disorder, its rates are sensitive to distortion by factors such as change in the underlying population structure, change in diagnostic habits, administrative inaccuracies when recording first admission or contact data and change in the organisation and delivery of psychiatric services (Kendell et al, 1993). In this study we set out to take account of these potential confounders and report servicebased incidence rates for schizophrenia in Dumfries and Galloway in south-west Scotland over the 20-year period 1979-1998.

\section{METHOD}

\section{Case identification}

Dumfries and Galloway is a geographically well-defined area in south-west Scotland. It has a stable population of around 147000 , of whom $99.5 \%$ are White. Psychiatric services are provided via one health trust and there is little or no private health care.

We identified all patients in contact with the psychiatric services between the years 1979 and 1998 who were given a clinical diagnosis of one of a broad range of psychotic disorders. During this period two different versions of the International Classification of Diseases (ICD) were used to code cases formally: ICD-9 (World Health Organization, 1978) from 1979 to
March 1996, and ICD-10 (World Health Organization, 1992) thereafter. We reviewed all cases with a diagnosis of schizophrenia (295, F20), schizoaffective disorder (295.6, F25), delusional disorder (297, F22), mania (296.0, 296.2, 296.4, F30, F31.1, F31.2, F31.6), acute, transient or unspecified psychotic disorders (298, F28, F29) or drug-induced disorders (292.1, F12.5, F16.6, F19.5, F12.75, F16.75, F19.75).

This list of possible cases was derived from two sources: data for all in-patients held centrally by the Information and Statistical Division of the Scottish Office (ISD); and locally held registers of outpatients, domiciliary visits and 'out of hours' referrals. Cases were excluded if they had presented with a psychotic episode before 1979 or were not resident in Dumfries and Galloway at the time of their presentation. The case records (including medical, nursing, social work and occupational therapy notes and all correspondence) of the remaining patients with firstepisode psychosis were examined and the Operational Checklist for Psychotic Disorders (OPCRIT) (McGuffin et al, 1991) was completed. The OPCRIT is a symptom checklist containing 90 items, which explores aspects of the mental state examination and the psychiatric history. It has a glossary of clear and explicit descriptions of each constituent item of psychopathology and instructions for coding them. It was designed with case note review in mind. Two of the authors (J.A., G.M.) completed the OPCRIT for the year following the initial presentation. This allowed us to compare each patient using similar information, irrespective of year of presentation. Case notes were examined in random order of date of presentation and raters were blind to the clinically coded diagnosis. There was good interrater reliability for ICD diagnosis of schizophrenia: $\kappa=0.79$ $(P=0.004)$. The OPCRIT checklist was then used to generate ICD-10 and DSM-IV (American Psychiatric Association, 1994) diagnoses via the associated computer algorithm OPCRIT 3.4.

\section{General population data (population at risk)}

The General Register Office of Scotland provided detailed population data for the region. Census data for the years 1971, 1981 and 1991, stratified by age and gender, were provided, and population estimates for the intermediate years were 
interpolated. The under-enumeration in Dumfries and Galloway was estimated at around $1 \%$.

\section{Statistical analyses}

Analyses were carried out in four blocks of 5 years: $1979-83,1984-88,1989-93$ and 1994-98. Incidence rates were calculated, stratified by age and gender, for the years 1979-83. These rates were used as our reference (standard) population rates. Using the indirect standardisation method, we applied this rate to the population structure in the subsequent year blocks. This allowed us to determine the number of expected cases. By dividing the number of cases actually observed by the number of cases expected, we calculated the standardised incidence ratio (SIR). Summary rate ratio linear trend, indicating the summary increase in risk with each consecutive time period, and gender modification effects were calculated using the Poisson Regression Procedure from the STATA program (Stata Corporation,
1999). These analyses were carried out for those patients receiving a clinical diagnosis of schizophrenia: OPCRIT-generated research diagnoses of ICD-10 and DSM-IV schizophrenia, as well as DSM-IV schizophrenia and schizoaffective disorder combined.

\section{RESULTS}

The number of patients with any one of the inclusion diagnoses in contact with the service during the 20-year period was 1460. After excluding cases with a previous psychotic episode prior to 1979 , or whose home address was outside the catchment area, there were 464 cases for which the OPCRIT was completed. There was a monotonic decline (the incidence for each year was less than or equal to the previous one) over the 20-year period in the rates for clinically diagnosed schizophrenia. This was statistically significant. The summary rate ratio trend was 0.77 (95\% confidence

Table I Standardised incidence ratios (SIR)

\begin{tabular}{|c|c|c|c|c|}
\hline Years & No. of observed cases & No. of expected cases & SIR & $95 \% \mathrm{Cl}$ \\
\hline \multicolumn{5}{|c|}{ Using clinical diagnosis of schizophrenia } \\
\hline 1979-83 & 75 & 75 & 1.00 & $0.79-1.25$ \\
\hline $1984-88$ & 49 & 78.75 & 0.622 & $0.46-0.82$ \\
\hline $1989-93$ & 47 & 81.54 & 0.576 & $0.42-0.77$ \\
\hline $1994-98$ & 32 & 74.65 & 0.429 & $0.29-0.60$ \\
\hline \multicolumn{3}{|c|}{ Summary rate ratio linear trend } & 0.77 & $0.68-0.88$ \\
\hline \multicolumn{5}{|c|}{ OPCRIT-derived ICD-I0 schizophrenia } \\
\hline $1979-83$ & 62 & 62 & 1.000 & $0.77-1.28$ \\
\hline $1984-88$ & 57 & 65.31 & 0.873 & $0.66-1.13$ \\
\hline $1989-93$ & 46 & 67.95 & 0.677 & $0.49-0.90$ \\
\hline $1994-98$ & 62 & 63.40 & 0.978 & $0.75-1.25$ \\
\hline \multicolumn{3}{|c|}{ Summary rate ratio linear trend } & 0.98 & $0.87-1.10$ \\
\hline \multicolumn{5}{|c|}{ OPCRIT-derived DSM-IV schizophrenia } \\
\hline $1979-83$ & 29 & 29 & 1.000 & $0.67-1.44$ \\
\hline $1984-88$ & 24 & 30.58 & 0.785 & $0.50-1.17$ \\
\hline $1989-93$ & 32 & 31.79 & 1.007 & $0.69-1.42$ \\
\hline $1994-98$ & 27 & 29.48 & 0.916 & $0.60-1.33$ \\
\hline \multicolumn{3}{|c|}{ Summary rate ratio linear trend } & 1.01 & $0.85-1.19$ \\
\hline \multicolumn{5}{|c|}{ OPCRIT-derived DSM-IV schizophrenia and schizoaffective disorder } \\
\hline 1979-83 & 52 & 52 & 1.000 & $0.75-1.31$ \\
\hline $1984-88$ & 53 & 54.58 & 0.971 & $0.73-1.27$ \\
\hline $1989-93$ & 45 & 56.51 & 0.796 & $0.58-1.06$ \\
\hline 1994-98 & 51 & 52.01 & 0.981 & $0.73-1.29$ \\
\hline \multicolumn{3}{|c|}{ Summary rate ratio linear trend } & 0.98 & $0.86-I . I I$ \\
\hline
\end{tabular}

interval (CI) 0.68-0.88). However, using OPCRIT-generated diagnoses there was no significant difference in the rates over time, irrespective of the diagnostic system applied. The summary rate ratio linear trend for ICD-10 schizophrenia was 0.98 (95\% CI 0.87-1.10) and for DSM-IV schizophrenia it was 1.10 (95\% CI 0.85-1.19). As previous studies have analysed DSMIV schizophrenia and schizoaffective disorder combined, we did the same; the summary rate ratio trend was $0.89(95 \% \mathrm{CI}$ 0.86-1.11).

As recently published work has shown gender differences in the incidence of schizophrenia (De Alacron et al, 1990; Der et al, 1990), we examined the modification (interaction) effect of gender on the rates over consecutive time periods. There was no significant gender-time interaction in the models. For clinically diagnosed cases, gender-time interaction was 0.89 (CI 0.69-1.16) for OPCRIT ICD-10 schizophrenia it was 0.98 (CI $0.87-1.10$ ) and for OPCRIT DSM-IV schizophrenia it was 0.87 (CI 0.62-1.22).

\section{DISCUSSION}

\section{Methodological issues}

\section{Strength of the study design}

(a) This study was carried out in an area with a stable population and no significant ethnic minority groups.

(b) By identifying not only all admissions to in-patient care, but also all day patients, out-patients, domiciliary visits and informal out-of-hours contacts, we avoided possible confounding effects due to the changes in service delivery.

(c) By reviewing all contacts with the service, we identified patients given a diagnosis of a psychotic disorder at first psychiatric presentation and also those psychotic patients who had previous periods of psychiatric care for non-psychotic episodes. This gives the most representative sample of firstever psychosis (Driessen et al, 1998).

(d) Standardised rates were used to ensure that any change in the at-risk population has not led to spurious changes in the incidence rates reported.

(e) Reviewing the case notes of all contacts during the 20 years reduced distortion from inaccurate administrative coding of first contacts.

(f) Finally, any analyses of rates over a long time period will be susceptible to bias 
from gradually changing diagnostic practices, so that by using the OPCRIT checklist and computer algorithm we ensured a consistent diagnostic approach for all patients.

\section{Limitations of the study design}

There are a number of limitations to bear in mind, although we believe they are of minor importance. We report service-based rates for one geographical area. This could introduce bias if the referral patterns of general practitioners changed during the study. However, it is assumed in well-developed services in the UK that nearly all patients with schizophrenia come into contact with services sooner or later (Cooper et al, 1987; Kendell et al, 1993).

Moreover, case note reviews may not be the best way to make a diagnosis. However, OPCRIT has been used successfully in a number of clinical and epidemiological studies (Williams et al, 1996). The two raters in our study were specialist registrars in psychiatry with demonstrated good interrater reliability, and the case notes reviewed were comprehensive. There is, however, a small possibility that the contents of case notes have changed over the study period.

\section{Interpretation of the findings}

We have shown a decline in the first contact rates for clinically diagnosed schizophrenia. This is consistent with previous studies in Scotland (Eagles et al, 1988). There is however no such decline when ICD-10 and DSM-IV diagnostic systems are consistently applied to all cases of psychotic disorders.

The difference between rates for clinically diagnosed schizophrenia and the OPCRIT-generated rates suggests that changes in diagnostic habits have indeed operated to confound the reported rates. The OPCRIT procedure resulted in a number of cases who were clinically diagnosed as suffering from schizophrenia in the early years of the period studied being reallocated to a range of other diagnostic categories, whereas the reverse tended to take place in the later years. This must reflect an increasing hesitancy or caution in making a diagnosis of schizophrenia.

There are at least two possible reasons for this. First, clinicians may now consider reliability of diagnosis important and therefore be using more restrictive operational criteria (Crow, 1990). Second, there may

\section{CLINICAL IMPLICATIONS}

- The incidence of consistently diagnosed schizophrenia has remained constant.

- It is important that early assessment be carried out to ensure that diagnostic hesitancy does not result in delay in starting appropriate management.

- Caution is necessary when using routinely collected data to inform changes in health care provision and service development.

\section{LIMITATIONS}

- This is a service-based study.

- The data are for one geographical area only.

- The study is over a 20 -year period only.

J. ALLARDYCE, MRCPsych, G. MORRISON, MRCPsych, R. G. McCREADIE, FRCPsych, Department of Clinical Research, Crichton Royal Hospital, Dumfries; J.VAN OS, PhD, European School of Neurosciences, Maastricht University, The Netherlands; J. KELLY, MRCP, R. M. MURRAY, FRCPsych, Institute of Psychiatry, London

Correspondence: Dr J. Allardyce, Department of Clinical Research, Crichton Royal Hospital, Dumfries DGI 4TG, Scotland, UK. Tel: +44 1387 244000; Fax: +44 I387 257735;

e-mail: j.allardyce@clinmed.gla.ac.uk

(First received 24 August 1999, final revision 20 December 1999, accepted 21 December 1999)

be an increasing reluctance at the initial presentation to make a diagnosis with such profound prognostic implications without first reviewing all the possible differential diagnoses. That clinicians may now be using narrower criteria for diagnosing schizophrenia has been recognised in several studies (Parker et al, 1985; MunkJørgensen, 1986; Kendell et al, 1993); our findings strongly support this assertion.

The findings of this study are at odds with most of the recently published work investigating trends over time in the incidence of schizophrenia. However, other studies which have closely considered diagnostic difficulties in their methodology have failed to show the magnitude of decline reported in studies depending on routinely collected data (Castle et al, 1991; Brewin et al, 1997). Also, the Scottish ageperiod-cohort study of Takei et al (1996) showed that most of the decline in the incidence of schizophrenia could be accounted for by changes at or near the time of diagnosis rather than by aetiological factors operating early in life.

We conclude that changes in diagnostic practice have resulted in declining rates of clinically diagnosed schizophrenia in the area we have studied. Our results show no sustained fall in the incidence of consistently diagnosed cases of schizophrenia. In southwest Scotland we have found no evidence to support the view that schizophrenia is disappearing.

\section{ACKNOWLEDGEMENTS}

We are grateful to ISD and The General Register Office of Scotland for their help. Our thanks to Mrs M. Muirhead for her administrative assistance and the Stanley Foundation for their financial support.

\section{REFERENCES}

American Psychiatric Association (1994) Diagnostic and Statistical Manual of Mental Disorders (4th edition) (DSM-IV). Washington, DC: APA.

Brewin, J., Cantwell, R., Dalkin, T., et al (1997) Incidence of schizophrenia in Nottingham. A comparison of two cohorts, 1978-80 and 1992-94. British journal of Psychiatry, I7I, 140-144.

Castle, D., Wessely, S., Der, G., et al (1991) The incidence of operationally defined schizophrenia in Camberwell, 1965-84. British Journal of Psychiatry, 159 790-794. 
Cooper, J. E., Goodhead, D., Craig, T., et al (1987) The incidence of schizophrenia in Nottingham. British Journal of Psychiatry, 15I, 619-626.

Crow, T. J. (1990) Trends in schizophrenia. Lancet, 335 851

De Alacron, J., Seagroatt, V. \& Goldacre, M. (1990) Trends in schizophrenia. Lancet, 335, 513-516.

Der, G., Gupta, S. \& Murray, R. M. (1990) Is schizophrenia disappearing? Lancet, 335, 513-516.

Driessen, G., Gunthner, N., Bak, M., et al (1998)

Characteristics of early- and late-diagnosed

schizophrenia: implications for first episode studies.

Schizophrenia Research, 33, 27-34.

Eagles, J. M. \& Whalley, L. J. (1995) Decline in the diagnosis of schizophrenia among first admissions to Scottish mental hospitals from 1969-78. British Journal of Psychiatry, 146, 151-154.

_, Hunter, D. \& McCance, C. (1988) Decline in the diagnosis of schizophrenia among first contacts with psychiatric services, in north-east Scotland, 1969-1984. British Journal of Psychiatry, 152, 793-798.

Geddes, J. R., Black, R. J., Whalley, L. J., et al (1993) Persistence of the decline in the diagnosis of schizophrenia among first admissions to Scottish hospitals from 1969 to 1988. British Journal of Psychiatry 163, 620-626.

Jablensky, A. (1997) The 100-year epidemiology of schizophrenia. Schizophrenia Research, 28, III-125.

Joyce, P. R. (1987) Changing trends in first admissions and readmissions for mania and schizophrenia in New Zealand 1974 to 1984. Australian and New Zealand Journal of Psychiatry, 21, 82-86.

Kendell, R. E., Malcolm, D. E. \& Adams, W. (1993)

The problem of detecting changes in the incidence of schizophrenia. British Journal of Psychiatry, 162, 212-218.

McGuffin, P., Farmer, A., Harvey, l., et al (1991) A polydiagnostic application of operational criteria in studies of psychotic illness. Archives of General Psychiatry, 48, 764-770.

Munk-Jørgensen, P. (1986) Decreasing first-admission rates of schizophrenia among males in Denmark from 1970 to 1984. Changing diagnostic patterns? Acta Psychiatrica Scandinavica, 73, 645-650.

Parker, G., O’Donnell, M. \& Walter, S. (1985) Changes in the diagnoses of the functional psychoses associated with the introduction of lithium. British Journal of Psychiatry, 146, 377-382.

Stata Corporation (1999) STATA Statistical Software Release 6.0. College Station, Texas: Stata Corp.

Suvisaari, J. M., Haukka, J. K., Tanskanen, A. J., et al (1999) Decline in the incidence of schizophrenia in the Finnish cohorts born from 1954 to 1965. Archives of General Psychiatry, 56, 733-740.

Takei, N., Lewis, G., Sham, P. C., et al (1996) Ageperiod-cohort analysis of the incidence of schizophrenia in Scotland. Psychological Medicine, 26, 963-973.

Williams. J., Farmer, A. E., Archenneil, M., et al (1996) A multi-centre inter-rater reliability study using the OPCRIT computerised diagnostic system. Psychological Medicine, 26, 775-783.

World Health Organization (1978) The Ninth Revision of the International Classification of Diseases and Related Health Problems (ICD-9). Geneva: WHO.

- (1992) The Tenth Revision of the International Classification of Diseases and Related Health Problems (ICD-10). Geneva: WHO. 


\section{CHAPTER 3}

\section{Variation in the incidence of schizophrenia by place}

Allardyce, J., Boydell, J., van Os, J., Morrison, G., Castle, D., Murray, R.M., and McCreadie, R.G. 2001.

Comparison of the incidence of schizophrenia in rural Dumfries and Galloway and urban Camberwell.

British Journal of Psychiatry 179:335-9. 
Comparison of the incidence of schizophrenia in rural Dumfries and Galloway and

\section{urban Camberwell}

\author{
J. ALLARDYCE, J. BOYDELL, J. VAN OS, G. MORRISON, D. CASTLE, \\ R. M. MURR AY and R. G. MCCREADIE
}

\begin{abstract}
Background Being born or living in urban areas is associated with high rates of schizophrenia. However, few studies have compared the incidence in urban/ rural areas using the same methodology.
\end{abstract}

Aims To compare service-based incidence rates for schizophrenia in rural Dumfries and Galloway in south-west Scotland with urban Camberwell in south-east London.

Method Using Research Diagnostic Criteria diagnoses from the OPCRIT computer algorithm, we compared the incidence of schizophrenia over 12 years (1979-1984 and 1992-1997) using indirect standardisation techniques and Poisson regression modelling.

Results The incidence was $61 \%$ higher in urban Camberwell than in rural Dumfries and Galloway (standardised incidence ratio $(\mathrm{SIR})=1.61$; $95 \% \mathrm{Cl}=1.42-$ I.8I). There was no difference in incidence when we compared the White population in Camberwell with Dumfries and Galloway $(\mathrm{SIR}=1.12 ; 95 \% \mathrm{Cl}=0.86-1.43)$.

Conclusions The incidence of schizophrenia in urban Camberwell was higher than that in rural Dumfries and Galloway; the high incidence of nonWhites in Camberwell largely explains the urban/rural difference.

Declaration of interest This work was supported by The Stanley Foundation and The Gordon Small Trust.
Several recent studies have claimed that being born or brought up in cities is associated with an increased risk of schizophrenia (Lewis et al, 1992; Marcelis et al, 1998, 1999; Mortensen et al, 1999). A genuine association between the incidence of schizophrenia and urban dwelling would be profoundly important for the understanding of the aetiology of this disorder. Unfortunately, it is difficult to establish whether or not incidence really does vary, because it is hard to ensure that the conditions and methods of measurement that have been used in different studies are the same for all areas being compared (Freeman, 1994). We therefore report an urban/rural comparison of first contact rates for schizophrenia using consistently generated diagnoses in two well-defined catchment areas, namely rural Dumfries and Galloway in south-west Scotland and inner-city Camberwell in south-east London. We also assess the impact on this comparison of the significant numbers of non-White individuals living in Camberwell, who when resident in the UK are reported to be at a higher risk of schizophrenia than the White population (Harrison et al, 1988; Wessely et al, 1991; King et al, 1994; van Os et al, 1996).

\section{METHOD}

\section{Study areas}

Dumfries and Galloway is a rural area in south-west Scotland with a population of around $147000,99.6 \%$ of whom were classified as White in the 1991 census. It is 2460 square miles in area and has a population density of 60 people per square mile. The local economy is based primarily on agriculture and farming, although there is some tourism and a little light industry. A comprehensive community-based psychiatric service is provided via out-patient clinics throughout the region. These clinics and their associated community psychiatric teams provide a service integrated with primary care and social services. One inpatient facility, situated at the Crichton Royal Hospital in the market town of Dumfries (population of approximately $30000)$, serves the area.

Camberwell is an inner-city area in south-east London congruent with the southern portion of the Borough of Southwark. The 1991 census showed it to have a population of approximately 120000 , of whom some $75 \%$ are White. It has a population density of some 19000 persons per square mile, that is, 316 times that of Dumfries and Galloway. Its economy was based historically on manufacturing industries but now is mixed. It contains the wealthy area of Dulwich (approximately $30 \%$ of the Camberwell catchment area with $13 \%$ of the Camberwell population) but also a number of poor areas. Over the period of the study, its psychiatric service has been provided via the same services based around the Maudsley Hospital but, as in Dumfries and Galloway, the services have moved towards a sectorised integrated community service.

\section{Identification of cases}

In both areas, we identified all patients making their first contact with psychiatric services who received a clinical diagnosis of one of a broad range of psychotic disorders. During the study period two different versions of the International Classification of Diseases (ICD) were used for formal coding of the cases: ICD-9 (World Health Organization, 1978) between 1979 and March 1996; and ICD10 (World Health Organization, 1992) thereafter. We reviewed all cases with a clinical diagnosis of schizophrenia (295; F20), schizoaffective disorder (295.6; F25), delusional disorder (297; F22), mania (296.0, 296.2, 296.4; F30, F31.1, F31.2, F31.6), acute, transient or unspecified psychotic disorders (298; F28, F29) or drug induced disorders (292.1; F12.5, F16.6, F19.5, F12.75, F16.75, F19.75) for the years 1979-1984 and 1992-1997. The second period allowed us to carry out a White-only comparison using information specifically about ethnicity that had not been collected before the 1991 census. In Dumfries and Galloway, all patients in contact with the psychiatric services during the study years who were given any of the 
above clinical diagnoses were identified from two sources: data for all in-patient admissions held centrally by the Information and Statistical Division Scotland (ISD); and data from locally held registers of outpatient contacts, domiciliary visits and emergency referrals.

In Camberwell, the data collection was carried out in two parts. For the years 1979-1984, a list was generated from the Camberwell Cumulative Register for all patients who, within a year of presentation, were given one of the inclusion diagnoses (details given in Castle et al, 1991). Cases were identified for the period 19921997 by hand-searching the case notes of all patients contacting the community facilities during these years and also via computerised information systems for all admissions to in-patient wards. Patients were divided into White and non-White groups based on information in their case records.

In both areas, cases were excluded if they were not resident in the catchment area, had presented previously with a psychotic episode outwith the study period or had a clear organic cause for their symptoms. For the remaining patients their case records, including medical, nursing, social work and occupational therapy notes together with all correspondence, were examined and the Operational Checklist for Psychotic Disorders (OPCRIT; McGuffin et al, 1991) was completed for the year following presentation. The OPCRIT is a symptom checklist containing 90 items that explores aspects of the mental state examination and psychiatric history. It has a glossary of clear and explicit descriptions for each constituent item of psychopathology and instructions for coding them. It was designed with case-note review in mind.

Two psychiatrists (J.A. and G.M.) working independently rated the case notes in Dumfries and Galloway. Two psychiatrists rated the Camberwell case notes for the 1979-1984 cases in Camberwell (Castle et al, 1991); interrater reliability was shown previously to be high $(\kappa=0.82$ for a Research Diagnostic Criteria (RDC) diagnosis of schizophrenia). One psychiatrist (J.K.) worked independently in Camberwell to complete the OPCRIT for the years 1992-1997. Interrater reliability was good for all three raters (J.A., G.M. and J.K.): $\kappa=0.79$ for an RDC diagnosis of schizophrenia. The OPCRIT checklists then were used to generate RDC diagnoses
(Spitzer et al, 1978) via the associated computer algorithm.

\section{General population data}

Data for the general populations stratified by age and gender were obtained in Dumfries and Galloway via the General Register Office Scotland and in Camberwell via the Office of Population Censuses and Surveys (OPCS, 1991) and the London Research Centre (1997). Census data for 1971, 1981 and 1991 were provided as well as population projections for 1997; estimations of the population for the intermediate years were interpolated. The 1991 census recorded information about self-reported ethnicity.

\section{Statistical analysis}

We compared first contact rates (servicebased incidence rates) for OPCRIT-derived RDC schizophrenia in the two areas for the 12 years 1979-1984 and 1992-1997. First contact rates have been reported previously using different diagnostic criteria in Dumfries and Galloway for the periods 1979-1998 (Allardyce et al, 2000) and 1979-1984 in Camberwell (Castle et al, 1991). To compare the incidence in the two areas we firstly calculated incidence rates stratified by age and gender for Dumfries and Galloway. These rates then were used as our reference or standard population rates. Using the indirect standardisation method, in the STATA statistical program (Stata Corporation, 1999) we applied these rates to the population structure of Camberwell. This allowed us to determine the number of expected cases if the rates were the same in both areas. By dividing the actual observed cases by the expected cases, we calculated the standardised incidence ratio (SIR).

To complement this SIR analysis, we constructed Poisson regression models using the STATA statistical program. In the Poisson regression model, the log of the incidence of schizophrenia is the response (dependent) variable. We examined the effect of the explanatory (independent) variable - urban exposure (residence in Camberwell) - on the incidence of schizophrenia while adjusting for gender, age and time period in the model. The exponentiated regression coefficient is the incidence rate ratio (IRR) (i.e. the incidence rate in Camberwell divided by the rate for Dumfries and Galloway). Previously published work demonstrated a higher incidence of schizophrenia in urban areas, more marked in men than women and higher in the younger age groups (Marcelis et al, 1998; Takei et al, 1995). We have examined, therefore, the interaction of urban exposure with age and gender and the interaction of age with gender.

\section{RESULTS}

\section{Comparison of incidence}

In Dumfries and Galloway over the 12 years, 177 cases were identified who fulfilled the OPCRIT-generated RDC criteria for schizophrenia (narrow and broad); all were White. In Camberwell, 265 cases were identified who met the RDC criteria, of whom $128(50 \%)$ were White. When we compared the two areas using indirect standardisation, the SIR was 1.61 (95\% CI 1.42-1.81), that is, the risk of schizophrenia was estimated to be $61 \%$ higher in Camberwell (Table 1). Stratified analyses showed the SIR varying across different levels of gender, age and time period, suggesting that these factors modify the effect of urban Camberwell residence (Table 2).

Poisson regression modelling reflected the findings of the SIR analyses. The IRR for 'Camberwell urban exposure' was 1.63 (95\% CI 1.35-1.98), adjusted for age, year and gender. There was a statistically significant interaction between urban exposure and gender $(\mathrm{IRR}=0.67 ; 95 \%$ CI $0.46-0.99)$, that is, the risk of urban residence was greater for males. The statistically significant interaction between gender and age (IRR $=1.29$; 95\% CI 1.16-1.46) shows that the risk of schizophrenia in females in both areas increases with age.

\section{White-only comparison}

We then compared the Dumfries and Galloway population with the White population in Camberwell using indirect standardisation for the period 1992-1997; we found no statistically significant difference in the rates (Table 3). The Poisson regression model again was unable to demonstrate any conclusive difference in risk; the IRR was 1.14 (95\% CI 0.82 1.58). There were no statistically significant interactions in this model. 
Table I Standardised incidence ratio (SIR) by comparing Dumfries and Galloway (D+G) with the total population of Camberwell

\begin{tabular}{lccc}
\hline Subjects and age (years) & Reference rate in D+G & Camberwell population' & Cases expected \\
\hline Male, I5-24 & 0.00018 & 114225 & 20.97 \\
Male, 25-34 & 0.00019 & 134778 & 25.89 \\
Male, 35-44 & $0.000 I I$ & 95683 & 10.29 \\
Male, 45-54 & 0.00009 & 78256 & 7.30 \\
Male, 55-64 & 0.00012 & 74500 & 9.18 \\
Male, 65-74 & 0.00005 & 56444 & 2.85 \\
Male, $>75$ & 0.00010 & 28518 & 2.74 \\
Female, I5-24 & 0.00015 & 125442 & 18.75 \\
Female, 25-34 & 0.00015 & 149668 & 22.20 \\
Female, 35-44 & 0.00017 & 101344 & 17.02 \\
Female, 45-54 & 0.00006 & $8225 I$ & 5.16 \\
Female, 55-64 & 0.00007 & 79205 & 5.21 \\
Female, 65-74 & 0.00005 & 69796 & 3.63 \\
Female, $>75$ & 0.00022 & 59023 & 13.18 \\
Total expected & & & 164.37 \\
Total observed & & & 265 \\
SIR=I.6I2 (95\% Cl=I.42-I.8I) & & & \\
\hline
\end{tabular}

I. At risk population over the study period.

Table 2 Standardised incidence ratio (SIR) stratified by time period, gender and age (reference rates from Dumfries and Galloway)

\begin{tabular}{lcccc}
\hline & Observed cases & Expected cases & SIR & $95 \% \mathrm{Cl}$ \\
\hline $\begin{array}{l}\text { Stratified by time period } \\
\text { I979-1984 }\end{array}$ & $\mathrm{II5}$ & & & \\
1992-1997 & 150 & 86.94 & 1.32 & $1.09-1.59$ \\
Stratified by gender & & 78.14 & 1.92 & $1.62-2.25$ \\
Male & 152 & & & \\
Female & 113 & 76.60 & 1.98 & $1.68-2.33$ \\
Stratified by age (years) & & 88.66 & 1.28 & $1.05-1.53$ \\
$15-24$ & 82 & & & \\
$25-34$ & 77 & 30.35 & 2.70 & $2.15-3.35$ \\
$35-44$ & 34 & 35.20 & 2.19 & $1.73-2.73$ \\
$45-54$ & 17 & 24.47 & 1.39 & $0.96-1.94$ \\
$55-64$ & 22 & 20.21 & 0.84 & $0.49-1.34$ \\
$65-74$ & 9 & 19.49 & 1.13 & $0.71-1.71$ \\
$>75$ & 24 & 15.95 & 0.56 & $0.26-1.07$ \\
\hline
\end{tabular}

Table 3 Standardised incidence ratio (SIR) for the time period 1992-1997 (reference rate from Dumfries and Galloway)

\begin{tabular}{lcccc}
\hline & Observed cases & Expected cases & SIR & $95 \% \mathrm{Cl}$ \\
\hline All cases in Camberwell & 150 & 78.14 & 1.92 & $1.62-2.25$ \\
White cases only in Camberwell & 62 & 55.58 & 1.12 & $0.86-1.43$ \\
\hline
\end{tabular}

\section{DISCUSSION}

\section{Methodological considerations}

Potential sources of bias and confounding

Before interpreting our findings, we must first consider potential sources of bias. We have compared service-based rates but not community rates. This potentially could confound our results if there was any regional difference in help-seeking behaviour. In Ireland a higher threshold for seeking medical advice was shown for neurotic disorders in rural than urban areas, but this was not the case for psychotic disorders (Keatinge, 1987). The distance to the psychiatric hospital also has been reported to be related inversely to the admission rates (Jarvis, 1851). The effect is small, however, for severe psychiatric disorders (Davey \& Giles, 1979). The influence of distance on service contact for psychosis is not clear but may be a factor in the level of service utilisation in the relatively distributed population of Dumfries and Galloway, and could be a potential source of bias. We think that this is unlikely because out-patient clinics and community teams are distributed throughout this rural area.

A variation in referral patterns of general practitioners to the different services also could confound our results. However, both areas are within the UK where, if it is suspected that a patient has a psychotic illness, general practitioners will almost invariably arrange a psychiatric referral (Kendell et al, 1993; Cooper et $a l$, 1987). It is conceivable that an occasional patient may be referred to a private practitioner; however, even if this did happen initially, it is very unlikely that someone with a psychotic disorder residing in either of the two areas would have no contact with the National Health Service over the study period. Potential bias due to variations in admission policies between the two centres was avoided by identifying all patients with a first-episode psychosis contacting the respective service, that is, not only those admitted to in-patient facilities but also out-patients, domiciliary visits and emergency contacts.

Case note review may not be an ideal way to make a diagnosis and we cannot exclude the possibility that case notes in the two centres contain different information, perhaps reflecting different clinical traditions. However, the phenomenological tradition in both centres stems from Mayer-Gross, 
who successively worked at the Maudsley Hospital in Camberwell and The Crichton Royal Hospital in Dumfries (Mayer-Gross et al, 1969). The case notes in both areas follow a semi-structured form and it is our experience that in both areas the notekeeping is of a high standard. The OPCRITgenerated RDC diagnosis for schizophrenia demonstrated good interrater reliability, thus ensuring that cases in both areas were diagnosed consistently using a well-established research classification system.

Finally, there was no significant underenumeration for Dumfries and Galloway in the 1991 census (estimated at around $1 \%)$, although under-enumeration may have occurred in Camberwell. It is impossible to know the full extent of this underenumeration and it is possible that there is some under-counting of the general population, particularly for the non-White groups. We have, however, employed population estimates that now have been corrected for this (London Research Centre, 1997).

\section{Case ascertainment}

For the period 1979-1984 there were minor differences in the case ascertainment in the two centres. Cases in Camberwell were identified by their diagnosis in the first year of presentation to psychiatric services, whereas in Dumfries and Galloway it was by first-event psychotic diagnosis. This may have relatively underestimated cases in Camberwell for this period. In the years 1992-1997 the case identification was the same in both centres. Therefore, different methods could not account for the findings in our comparison of White subjects. Owing to the small numbers in the White-only comparison, we were unable to examine interactions with age and gender, which may be masked by the summary measures in this comparison.

\section{Comparison of incidence}

We have shown a higher incidence of schizophrenia in urban Camberwell compared with rural Dumfries and Galloway. The risk for urban residence was greatest for males. This is consistent both with recently published work comparing urban/rural variations in (first) admission rates for schizophrenia (Systema, 1991; Peen \& Dekker, 1997) and with cohort studies, suggesting that being born or brought up in an urban environment is associated with an increased risk of schizophrenia (Lewis et al, 1992; Marcelis et al, 1998, 1999; Mortensen et al, 1999).

\section{White-only comparison}

We found no statistically significant difference in the incidence of schizophrenia when the White population in Camberwell was compared with those in Dumfries and Galloway.

This suggests that non-White individuals living in Camberwell account for a large proportion of the increased urban incidence that we have found. The ethnic minority population in Camberwell is a mixed one but the largest group - approximately $11 \%$ of the total population - comprise individuals who migrated from the Caribbean together with their children. There is considerable evidence from Camberwell and elsewhere that this population has an incidence of schizophrenia that is four- to sixfold higher than the rest of the British population. This appears to be more prominent in the children of people who have migrated (Harrison et al, 1988; Wessely et al, 1991; van Os et al, 1996).

Is it possible that recent studies suggesting an increased incidence of schizophrenia in urban areas reflect the different population compositions in urban and rural areas, with migrants and their offspring primarily living in inner-city areas (Herbert \& Johnston, 1976). This seems unlikely because the authors of the various cohort studies assert that their studies, in which an urban place of birth was associated with an increased risk of schizophrenia, were carried out in areas with relatively little external (i.e. from another country) migration.

Why, then, do our results in the White population differ from these cohort studies? First, there may be something atypical about Camberwell, because it contains a relatively rich and predominantly White area (Dulwich), with the non-White people tending to live in the poorer areas; thus, social class and wealth may confound the ethnic division. Second, perhaps it is those individuals in the inner-city who are most disadvantaged and alienated from mainstream society who have an increased incidence of schizophrenia. In south London, African-Caribbeans have taken over this role but it could be that in cities without Black immigrants the low social class Whites occupy this position. Finally, it may be that the cohort studies identified those with only relatively early-onset schizophrenia and missed the later-onset cases, whereas our study identified all first-episode cases of schizophrenia in all age groups in both areas. The onset of schizophrenia may be later in rural areas (Eaton, 1974). A longer follow-up period in the cohort studies might, therefore, have identified more rural cases and thus reduced the apparent urban/rural difference. Unfortunately we did not have the power to detect any age and gender interactions in our White population; larger studies should explore these possible interactions.

\section{ACKNOWLEDGEMENTS}

This study was supported by grants from The Stanley Foundation and The Gordon Small Trust. We are grateful to Jennifer Bishop at ISD, the General Register Office (Edinburgh) and the London Research Centre for their help.

\section{REFERENCES}

Allardyce, J., Morrison, G., McCreadie, R. G., et al (2000) Schizophrenia is not disappearing in south-west Scotland. British Journal of Psychiatry, 177, 38-4I.

Castle, D., Wessely, S., Der, G., et al (1991) The incidence of operationally defined schizophrenia in Camberwell, 1965-84. British Journal of Psychiatry, 159, 790-794.

Cooper, J. E., Goodhead, D., Craig, T., et al (1987) The incidence of schizophrenia in Nottingham. British Journal of Psychiatry, 15I, 619-626.

Davey, S. \& Giles, G. (1979) Spatial factors in mental health care in Tasmania. Social Science and Medicine, $\mathbf{1 3}$ 87-94.

Eaton, W. (1974) Residence, social class and schizophrenia. Journal of Health and Social Behaviour, 15, 289-299.

Freeman, H. (1994) Schizophrenia and city residence. British Journal of Psychiatry, 164 (suppl. 23), 39-50.

Harrison, G., Owens, D., Holten, A., et al (1988) A prospective study of severe mental disorder in Afro-Caribbean patients. Psychological Medicine, 18, 643-657.

Herbert, D. T. \& Johnston, R. J. (1976) Social Areas in Cities. Chichester: Wiley.

Jarvis, E. (185I) On the supposed increase of insanity. American Journal of Insanity, 8, 333-364.

Keatinge, C. (1987) Community factors influencing psychiatric hospital utilisation in rural and urban Ireland. Community Mental Health Journal, 23, 192-203.

Kendell, R. E., Malcolm, D. E. \& Adams, W. (1993) The problem of detecting changes in the incidence of schizophrenia. British Journal of Psychiatry, 162, 212-218.

King, M., Coker, E., Leavy, G., et al (1994) Incidence of psychotic illness in London: comparison of ethnic groups. BMJ, 309, III5-1119. 
Lewis, G., David, A., Andreasson, S., et al (1992) Schizophrenia and city life. Lancet, 340, 137-140.

London Research Centre (1997) Methodology of the Ethnic Group Projections 1997 Round. London: London Research Centre.

Marcelis, M., Navarro-Mateu, F., Murray, R., et al (1998) Urbanisation and psychosis: study of 1942-1978 birth cohorts inThe Netherlands. Psychological Medicine, 28. $87 \mid-879$.

_ , Takei, N. \& van Os, J. (1999) Urbanisation and psychosis: does the effect operate before or around the time of illness onset? Psychological Medicine, 29. 1197-1203.

Mayer-Gross, W., Slater, E. \& Roth, M. (1969)

Clinical Psychiatry (3rd edn). London: Baillière, Tindall \& Cassell.

McGuffin, P., Farmer, A., Harvey, l., et al (1991) A polydiagnostic application of operational criteria in studies of psychotic illness. Archives of General Psychiatry, 48, 764-770.

Mortensen, P. B., Carsten, B. P., Westergaard, T., et al (1999) Effects of family history and place and season of birth on the risk of schizophrenia. New England Journal of Medicine, 340, 603-608.

Office of Population Censuses and Surveys (1991) Population Estimates Unit, Census Crown Copyright. London: OPCS.

Peen, J. \& Dekker, J. (1997) Admissions rates for schizophrenia in the Netherlands: an urban/rural comparison. Acta Psychiatrica Scandinavica, 96 30I-305.

Spitzer, R. L., Endicott, J. \& Robins, E. (1978) Research diagnostic criteria: rationale and reliability. Archives of General Psychiatry, 36, 17-24.

Stata Corporation (1999) STATA Statistical Software Release 6.0. College Station, TX: StataCorp.

Systema, S. (1991) Social indicators and psychiatric admissions: a case register study in The Netherlands. Psychological Medicine, 2I, 177-184.

Takei, N., Sham, P. E. O. C., Glover, G., et al (1995) Schizophrenia: increased risk associated with winter and city birth - a case control study in 12 regions within England and Wales. Journal of Epidemiological and Community Health, 49, 106-109.

\section{CLINICAL IMPLICATIONS}

- Standardised incidence rates for schizophrenia are higher in urban Camberwell than in rural Dumfries and Galloway.

- The increased risk in urban Camberwell is greater in males than females.

- Non-White individuals living in Camberwell account for a large proportion of this demonstrated increased incidence.

\section{LIMITATIONS}

- This is a service-based study.

- We have compared only two discrete geographical areas.

- Relatively small numbers in the comparison of the White-only population of Camberwell with Dumfries and Galloway hinder the exploration of age and gender interactions in the model.

J. ALLARDYCE, MRCPsych, G. MORRISON, MRCPsych, R. G. McCREADIE, FRCPsych, Department of Clinica Research, Crichton Royal Hospital, Dumfries, UK; J.VAN OS, PhD, Department of Psychiatry and Neuropsychology, European School of Neurosciences, Maastricht University, Maastricht, The Netherlands; J. BOYDELL, MRCPsych, R. M. MURRAY, FRCPsych, Department of Psychological Medicine, Institute of Psychiatry, London, UK; D. J. CASTLE, MRCPsych, University of Western Australia, Perth, Western Australia

Correspondence: Dr J. Allardyce, Department of Clinical Research, Crichton Royal Hospital, Dumfries DGI 4TG, Scotland, UK. Tel: +44 1387 244000; fax: +44 I387 257735; e-mail: j.allardyce@clinmed.gla.ac.uk

(First received 3 July 2000, final revision 19 February 200I, accepted 7 March 200I)

van Os, J., Castle, D., Takei, N., et al (1996) Psychotic illness in ethnic minorities: classification from the 1991 census. Psychological Medicine, 26, 203-208.

Wessely, S., Castle, D., Der, G., et al (1991)

Schizophrenia and Afro-Caribbeans: a case-control study. British Journal of Psychiatry, 159, 795-80I.
World Health Organization (1978) The ICD-9 Classification of Mental and Behavioural Disorders. Geneva: WHO.

- (1992) The ICD-10 Classification of Mental and Behavioural Disorders. Geneva: WHO. 



\section{CHAPTER 4}

\section{Variation in the incidence of psychosis by area level social characteristics}

Allardyce, J., Gilmour, H., Atkinson, J., Rapson, J., Bishop, J and McCreadie, R.G. 2005.

Social Fragmentation, deprivation and urbanicity: Relationship to first -admission rates for psychoses .

British Journal of Psychiatry 187:401-406. 


\section{Social fragmentation, deprivation and urbanicity: relation to first-admission rates for psychoses}

\author{
J. ALLARDYCE, H. GILMOUR, J. ATKINSON, T. RAPSON, J. BISHOP \\ and R. G. MCCREADIE
}

\begin{abstract}
Background Social disorganisation, fragmentation and isolation have long been posited as influencing the rate of psychoses at area level. Measuring such societal constructs is difficult. A censusbased index measuring social fragmentation has been proposed.
\end{abstract}

Aims To investigate the association between first-admission rates for psychosis and area-based measures of social fragmentation, deprivation and urban/rural index.

\section{Method We used indirect standardisation methods and logistic regression models to examine associations of social fragmentation, deprivation and urban/rural categories with first admissions for psychoses in Scotland for the 5-year period 1989-1993.}

\section{Results Areas characterised by high social fragmentation had higher first-ever admission rates for psychosis independent of deprivation and urban/rural status. \\ There was a dose-response relationship between social fragmentation category and first-ever admission rates for psychosis. There was no statistically significant interaction between social fragmentation, deprivation and urban / rural index.}

Conclusions First-admission rates are strongly associated with measures of social fragmentation, independent of material deprivation and urban/rural category.

Declaration of interest. None.
Geographical studies show that populations of areas with greater material deprivation have higher rates of psychoses (Dauncey et al, 1993; Harrison et al, 1995; Harvey et al, 1996; Boardman et al, 1997) and that urban areas have a greater risk of psychoses compared with rural areas (Lewis et al, 1992; Marcelis et al, 1998; Mortensen et al, 1999; Allardyce et al, 2000). Also, the association with deprivation may apply to urban but not rural areas (Thornicroft et al, 1993). Although strong associations are shown for areabased indices of deprivation and psychosis they may not fully describe the ecological relationship. Faris \& Dunham (1939) and Hare (1956) recognised the importance of social disorganisation, fragmentation and isolation in the onset of mental disorders and more recent reports support this proposition (van Os et al, 2000). Congdon (1996) has proposed a census-based index measuring anomie (social fragmentation) and we used this to examine the relative impact of social fragmentation, material deprivation and urbanicity/rurality on firstadmission rates to hospital for psychosis, using a Scottish national data-set for the period 1989-1993.

\section{METHOD}

To allow chronological matching, we identified cases in which hospital admission occurred during the period 1989-1993, and used information on social factors from the 1991 national census to calculate categories of deprivation, social fragmentation and urban/rural classification.

\section{Case identification}

The Scottish Office Information and Statistics Division collates in-patient activities in Scottish hospitals. All psychiatric facilities return a form (SMR04) for each patient after an in-patient stay; this form provides both demographic and diagnostic information. During the period 19891993 the main diagnoses were coded according to ICD-9 (World Health Organization, 1978). The record linkage section of the Information and Statistics Division identified for this period all cases with a discharge diagnosis of schizophrenia (code 295), schizoaffective disorder (295.6), delusional disorder (297), mania (296.0, 296.2, 296.4), acute, transient or unspecified psychotic disorder (298) or drug-induced disorder (292.1), in which the patient had had no previous admission (in Scotland) for any of the above psychotic diagnoses. We excluded patients over the age of 64 years (as there is likely to be significant diagnostic difficulty with elderly people) and those without a permanent address in Scotland.

\section{Population at risk}

The population of Scotland was determined at around 5 million (all age groups) throughout the study period. At the 1991 census only $2.8 \%$ of the population had been born outside of the UK. The General Register Office of Scotland provided detailed population data for the 5 -year period stratified by age, gender and postcode sector (an area with an average population of 5000 considered of sufficient size to provide fairly reliable rates for health events; Carstairs \& Morris, 1991).

\section{Area-based measures}

Area-based measures were calculated for every postcode.

\section{Social fragmentation}

We calculated social fragmentation using information from the 1991 census on mobility in the previous year, number of privately rented households, single-person households and number of unmarried persons (Congdon, 1996). The social fragmentation index for each postcode sector was calculated by adding the $z$ scores (the number of standard deviations above or below the population mean when the underlying distribution is normal) for each of the four characteristics. The scores ranged from -4.8 to 33.79 . For the purpose of the analysis presented here we collapsed the index into categories, created by quartiles. However, because there was a strong positive skew in the distribution of the social fragmentation scores, the upper quartile was divided at the 90th percentile, 
creating five categories - category 1 being the most socially cohesive area and 5 the most socially fragmented.

\section{Material deprivation}

Material deprivation was measured using Carstairs scores, the indicators routinely used in Scotland (Carstairs \& Morris, 1991). Carstairs scores correlate highly with other commonly used indices of deprivation (Townsend 0.96, Jarman 0.83). The scores were calculated using the 1991 census data for overcrowding, male unemployment, low social class and no car. The postcode sector scores range from -8.5 (most affluent) to 12.8 (most deprived). The deprivation scores for each postcode sector are transformed routinely into categories, using pre-defined cut-off scores, which range from category 1 (most affluent) to 7 (most deprived) (McLoone, 1995).

\section{Urban/rural index}

We measured the urban/rural index using data from the 1991 census and the official Scottish classification (Carstairs \& Morris, 1991). The degree of urbanicity is calculated for each postcode sector by adding to the population total the population of each directly adjacent neighbourhood: category 1 is most urban, and category 5 and 6 are the most rural.

\section{Analysis}

Using the indirect standardisation method we calculated standardised (first) admission ratios by category of social fragmentation, deprivation and urban/rural classification. For each (10-year) age and gender band we used both the national first-admission rates and the rates for stratum 1 of each social characteristic as the reference. Next, we calculated the age/gender-adjusted admission rates for first-ever psychosis for each postcode sector in order to model their dependence on social fragmentation, adjusting for deprivation category and urban/rural index. As there was evidence of overdispersion in the admission rates they could not be adequately modelled. To overcome this the admission rate distribution was dichotomised into the high-rate quintile (rate lies within the top 20\% of admission rates) and the remainder. Logistic regression analysis was used to determine whether social fragmentation, deprivation and urban/rural index were independently associated with first admission rates classified as 'high'. A sensitivity analysis was carried out using the 75 th percentile and the 85 th percentile to check whether the actual cut-off point used to define high rates was critical in the interpretation of the results.

\section{RESULTS}

The SMR04-linked data-set contained 5858 cases eligible for inclusion; $20(0.34 \%)$ had missing or unknown postcodes and 5838 cases were therefore entered into the analysis. Tables 1-6 show the standardised admission ratios categorised according to fragmentation (Tables 1-2), deprivation category (Tables 3 and 4) and urban/rural level (Tables 5 and 6). The odds ratios that social fragmentation, deprivation and urbanicity measures predict standardised first-ever psychosis admission rates are shown in Table 7.

Univariate analysis showed a very significant association for social fragmentation category, deprivation category and urban/rural index with 'high rate' postcode sectors. In the adjusted model this very significant association remained for social fragmentation only. There was no significant interaction between social fragmentation, deprivation and the urban/rural index in the model.
The model is a good fit to the data (Hosmer-Lemeshow test, $P=0.78$ ) and the results are essentially the same using the 75th and 85th percentiles as the cut-off for defining high admission rate.

\section{DISCUSSION}

\section{Main findings}

\section{Social fragmentation}

This study suggests that areas characterised by high social fragmentation have higher first-ever admission rates for psychosis independent of deprivation and urban/rural status. There is a clear monotonic gradient in the relationship between social fragmentation and first-ever admission rates for psychosis. The odds that a postcode from the most fragmented category has a high admission rate is 12.8 (95\% CI 5.7-28.9) times the odds for a postcode from the least fragmented areas.

We are unaware of any recently published work exploring rates of psychosis with composite measures of social fragmentation. Faris \& Dunham (1939) recognised urban areas with high rates of psychiatric morbidity to be characterised by social disintegration, excessive residential mobility, ethnic conflict, communication breakdown and lack of consensus. Social isolation leading to mental health inequality was suggested by Hare (1956), who found an

Table I Standardised admission ratios for social fragmentation (using national rates as reference)

\begin{tabular}{lccccc}
\hline Social fragmentation category' & Expected cases & Observed cases & \multicolumn{2}{c}{ Standardised admission ratio } \\
\cline { 4 - 5 } & $n$ & $n$ & & Ratio & $(95 \% \mathrm{Cl})$ \\
\hline 1 & & & 1434 & 0.77 & $(0.73-0.81)$ \\
2 & 1858.15 & 1466 & 0.91 & $(0.87-0.96)$ \\
3 & 1605.92 & 1219 & 1.06 & $(1.00-1.06)$ \\
4 & 1149.25 & 932 & 1.42 & $(I .33-1.51)$ \\
5 & 655.31 & 787 & 1.60 & $(I .49-1.71)$ \\
\hline
\end{tabular}

I. Category I, most socially cohesive; category 5 , most socially fragmented.

Table 2 Standardised admission ratios for social fragmentation (using category I as reference rate)

\begin{tabular}{lccccc}
\hline Social fragmentation category' & Expected cases & Observed cases & \multicolumn{2}{c}{ Standardised admission ratio } \\
\cline { 3 - 5 } & $n$ & $n$ & Ratio & $(95 \% \mathrm{Cl})$ \\
\hline 1 & & & 1 & - \\
2 & 1434 & 1434 & 1.18 & $(1.12-1.24)$ \\
3 & 1243.16 & 1466 & 1.37 & $(1.29-1.44)$ \\
4 & 890.97 & 1219 & 1.84 & $(1.78-1.90)$ \\
5 & 576.9 & 932 & 2.09 & $(1.95-2.24)$ \\
\hline
\end{tabular}

I. Category I, most socially cohesive; category 5 , most socially fragmented. 
Table 3 Standardised admission ratios for deprivation (using national rates as reference)

\begin{tabular}{lccccc}
\hline Deprivation category' & Expected cases & Observed cases & \multicolumn{2}{c}{ Standardised admission ratio } \\
\cline { 4 - 5 } & $n$ & $n$ & & Ratio & $(95 \% \mathrm{Cl})$ \\
\hline 1 & & & 0.69 & $(0.60-0.78)$ \\
2 & 328.11 & 225 & 0.82 & $(0.76-0.88)$ \\
3 & 806.51 & 661 & 0.87 & $(0.82-0.92)$ \\
4 & 1266.07 & 1101 & 0.94 & $(0.89-0.99)$ \\
5 & 1457.97 & 1377 & 1.12 & $(1.05-1.19)$ \\
6 & 880.59 & 985 & 1.33 & $(1.24-1.41)$ \\
7 & 633.65 & 840 & 1.68 & $(1.55-1.80)$ \\
\hline
\end{tabular}

I. Category I, most affluent; category 7, most deprived.

ecological correlation with single-person households in Bristol and rates of first admission for schizophrenia. A more recent study has shown area-based measures of single and divorced residents to be associated with higher first-contact rates for psychosis, independent of a number of neighbourhood social and demographic characteristics and individual measures of age, gender and marital status (van Os et al, 2000). Thornicroft et al (1993) demonstrated that in urban areas the proportion of unmarried people and the proportion of people living alone were strongly correlated with admissions for psychosis.

\section{Material deprivation}

Our results are consistent with previous work demonstrating an association between admissions for psychosis and populationbased measures of material deprivation (Harrison et al, 1995; Boardman et al, 1997; Koppel \& McGuffin, 1999). We show this association to hold for firstadmission data also. Adjustment for social fragmentation and urbanicity in our logistic model weakened the association. Although

Thornicroft et al (1993) found an association between deprivation and service utilisation rates for psychosis in south Verona (an urban area), there was no relationship in the same study with deprivation in the rural area of Portogruaro. However, we found no interaction of deprivation and urban/rural terms in our model, i.e. the effect of deprivation does not vary across urban/rural categories. We studied all postcodes in Scotland, allowing examination of rural areas heterogeneous for material and social deprivation. The 11 rural districts in the Italian study might have been too similar to detect any association in this relatively small area.

\section{Urban/rural variation}

The urban/rural differences in admission rates for psychoses demonstrated in this study have been well documented in previous studies (Marcelis et al, 1998; Mortensen et al, 1999; Allardyce et al, 2001). However, we have not shown a statistically significant variation in admission rates with urbanicity after adjustment for social fragmentation and deprivation. It is therefore possible that deprivation and
Table 4 Standardised admission ratios for deprivation (using category I as reference rate)

\begin{tabular}{lccccc}
\hline Deprivation category' & Expected cases & Observed cases & \multicolumn{2}{c}{ Standardised admission ratio } \\
\cline { 4 - 6 } & $n$ & $n$ & & Ratio & $(95 \% \mathrm{Cl})$ \\
\hline 1 & 225 & 225 & 1 & - \\
2 & 558.09 & 661 & 1.18 & $(1.09-1.27)$ \\
3 & 880.35 & 1101 & 1.25 & $(1.18-1.32)$ \\
4 & 1019.05 & 1377 & 1.35 & $(1.28-1.45)$ \\
5 & 618.00 & 985 & 1.59 & $(1.49-1.69)$ \\
6 & 478.33 & 840 & 1.76 & $(1.64-1.87)$ \\
7 & 272.70 & 649 & 2.38 & $(2.20-2.56)$ \\
\hline
\end{tabular}

I. Category I, most affluent; category 7 , most deprived. social fragmentation are important explanatory factors in the urban effect seen in previous studies.

\section{Methodological considerations}

Data-set and admission rate calculations

The SMR04 data-set provides national, comprehensive $(100 \%$ coverage $)$ information for in-patient care over three decades in Scotland. The usefulness of such a data-set depends on the accuracy of its information, and despite earlier criticism (Kendrick \& Clarke, 1993) the quality of the SMR04 data is now considered good (Harley \& Jones, 1996). The quantity of the data should reduce the effect of variation in local coding practices, but some variation due to regional differences may remain; we have used the broad diagnostic category 'psychosis' to calculate first-ever admission rates as it is likely to have the greatest diagnostic consistency (Allardyce et al, 2001).

We examined admission rates from the fine-grain level of postcode sectors and have offset the possible disadvantage of low numbers by taking admissions over a 5 -year period and using a dichotomised outcome measure. Admission rates reflect only the met demand for in-patient care, and it is possible that areas with better community facilities and day hospital provision will use fewer beds; however, this assumption is not supported for psychosis (Jarman et al, 1992; Flannigan et al, 1994). In Scotland during the period of study, there was no specific day care alternative to admission for people with severe mental illness.

\section{Measures of area-based exposures}

The area-based measures of deprivation and social fragmentation were generated from aggregation of census-based variables. The census is the only source of objective and uniform data for the entire population and therefore any proxy measure is constrained by the data available from it. As a sound conceptual base for either social fragmentation (social cohesion) or deprivation is lacking, there is no absolute underlying theory in the selection of variables used in the aggregate scores (Carr-Hill, 1988). The demographic factors we used to measure fragmentation namely non-married adults, one-person households, population turnover and private renting - may not in themselves be 
Table 5 Standardised admission ratios categorised by urban/rural index (using national rates as reference)

\begin{tabular}{lccccc}
\hline Urban/rural category' & Expected cases & Observed cases & \multicolumn{2}{c}{ Standardised admission ratio } \\
\cline { 3 - 5 } & $n$ & $n$ & & Ratio & $(95 \% \mathrm{Cl})$ \\
\hline 1 & & & 1.21 & $(\mathrm{I} .16-\mathrm{I} .27)$ \\
2 & 1553.33 & 1883 & 1.24 & $(\mathrm{I} .17-\mathrm{I} .32)$ \\
3 & 970.77 & 1208 & 0.90 & $(0.85-0.94)$ \\
4 & 1828.30 & 1641 & 0.78 & $(0.73-0.82)$ \\
5,6 & 1245.77 & 966 & 0.86 & $(0.72-1.01)$ \\
\hline
\end{tabular}

I. Category I, most urban; category 6, most rural.

Table 6 Standardised admission ratios categorised by urban/rural index (using category I as reference rate)

\begin{tabular}{lcccc}
\hline Urban/rural category' & Expected cases & Observed cases & \multicolumn{2}{c}{ Standardised admission ratio } \\
\cline { 3 - 5 } & $n$ & $n$ & & \\
& & & Ratio & $(95 \% \mathrm{Cl})$ \\
\hline 1 & 1883 & 1883 & 1 & - \\
2 & 1177.68 & 1208 & 1.03 & $(0.97-1.08)$ \\
3 & 2216.92 & 1641 & 0.74 & $(0.72-0.76)$ \\
4 & 1512.88 & 966 & 0.64 & $(0.60-0.68)$ \\
5,6 & 188.62 & 140 & 0.74 & $(0.62-0.87)$ \\
\hline
\end{tabular}

I. Category I, most urban; category 6, most rural.

Table 7 Odds ratios for area measures of social fragmentation, deprivation and urbanicity predicting 'high' first-admission rates for psychosis

\begin{tabular}{|c|c|c|c|c|c|}
\hline & \multicolumn{2}{|c|}{ Unadjusted } & \multicolumn{3}{|c|}{ Adjusted } \\
\hline & Odds ratio & $P$ & Odds ratio & $P$ & $95 \% \mathrm{Cl}$ \\
\hline Social fragmentation category' & & $<0.001$ & & $<0.001$ & \\
\hline I & 1.00 & & 1.00 & & \\
\hline 2 & 4.10 & & 4.29 & & $2.04-9.05$ \\
\hline 3 & 5.09 & & 5.34 & & $2.52-11.32$ \\
\hline 4 & 11.38 & & 9.38 & & $4.30-20.46$ \\
\hline 5 & 20.37 & & 12.84 & & $5.7 I-28.88$ \\
\hline Deprivation category ${ }^{2}$ & & $<0.00 \mathrm{I}$ & & 0.014 & \\
\hline 1 & 1.00 & & 1.00 & & \\
\hline 2 & 1.71 & & 1.44 & & $0.44-4.76$ \\
\hline 3 & 2.16 & & 2.03 & & $0.64-6.43$ \\
\hline 4 & 2.88 & & 2.43 & & $0.78-7.55$ \\
\hline 5 & 4.16 & & 3.40 & & $1.06-10.88$ \\
\hline 6 & 8.31 & & 4.58 & & $1.39-15.09$ \\
\hline 7 & 15.80 & & 5.29 & & $1.49-18.75$ \\
\hline Urban/rural category ${ }^{3}$ & & $<0.001$ & & 0.177 & \\
\hline I & 1.00 & & 1.00 & & \\
\hline 2 & 0.84 & & 0.93 & & $0.50-1.73$ \\
\hline 3 & 0.31 & & 0.60 & & $0.32-1.09$ \\
\hline 4 & 0.21 & & 0.43 & & $0.22-0.85$ \\
\hline 5 & 0.35 & & 0.51 & & $0.21-1.26$ \\
\hline 6 & 0.55 & & 0.62 & & $0.29-1.32$ \\
\hline
\end{tabular}

I. Category I, most socially cohesive; category 5 , most socially fragmented.

2. Category I, most affluent; category 7 , most deprived.

3. Category I, most urban; category 6, most rural. valid indicators of social fragmentation, or may adequately measure social fragmentation in some areas but not in others. For example, in urban areas the combination of young, single people living in non-family households may not measure disorganised communities but rather communities with young professionals or students (Congdon, 1996). Similarly, the individual census variables chosen may reflect deprivation in some areas better than others. For example, overcrowding is an almost exclusively urban phenomenon and is likely to be irrelevant in identifying deprivation in rural areas. Lack of a car may be an indicator of deprivation in an urban context, but possession of a car in rural areas may be almost a necessity. In rural areas car ownership is highly correlated with remoteness rather than socio-economic group (Midwinter et al, 1988).

As a population census is only performed every 10 years in the UK, it is more than possible that an area's characteristics may change during this time with obviously no concomitant change in the census aggregated score. However, as we have analysed data from the period 1989-1993, area-based measures and admission data are chronologically matched.

There is no universally accepted definition of 'rural'. Characteristics may include open spaces, green scenery, agricultural activities, remoteness and lack of people. Most published work has used quantitative definitions of 'urban/rural', but as there is no point on the continuum from large agglomerations to small clusters or scattered dwellings where 'urban' disappears and 'rural' begins, the division between urban and rural population will always be arbitrary. Despite these methodological limitations, area-based measures are increasingly used in public health research and practice (Smith \& Hart, 1999).

It is unlikely that any area, however small, will be totally homogeneous for exposure levels of deprivation, social fragmentation and urban/rural characteristics. This is especially so if the geographical categorisation is logistical, as in postcode sectors. However, in Scotland postcode sectors are more socioculturally homogeneous than elsewhere in the UK (Reijneveld $e t a l$, 2000). Although area-based measures may not apply equally to all individuals within an area, all these individuals are exposed to living in a neighbourhood with (for example) low social fragmentation or high 
deprivation levels. Social characteristics such as social fragmentation in our society are likely to have profound effects on health and yet are incompletely captured and described by individual approaches to measurement.

\section{Ecological study design}

This is an area-based study comparing groups rather than individuals, allowing the ecological effects of constructs conceptualised at the group/area level, such as social fragmentation, deprivation and urban/rural categories, to be demonstrated. A study with both individual and ecological information would allow us to look at the person-environment interaction, which would be very informative; however, data at the individual level were not available.

Finally, standardised (first) admission ratios may not be the best way of comparing morbidity in different geographical areas, because each subgroup is adjusted to a different standard. These ratios are, however, fairly robust with respect to the violation of the assumption of proportionality (Court $\&$ Cheng, 1995). This method has the advantage over direct standardisation in that it has a smaller variance. The wide confidence intervals that would be generated by other methods of standardisation would cause difficulty in interpreting the results and might be misleading. We have complemented the standardised admission ratios analysis with a logistic regression model.

\section{Clinical implications}

Association does not imply causality. Living in a socially fragmented deprived area may precipitate a first episode of psychosis (social causation hypothesis); alternatively, individuals predisposed to psychosis may drift into or out of or be left in areas (social selection). It is also possible that some other variable might be confounding the effect and the deprivation and social fragmentation indices are simply proxy measures. Our study suggests, whatever the underlying mechanism, that both material deprivation and social fragmentation are likely to influence first-admission rates for psychosis at area level. However, we found social fragmentation to have the greatest effect. The observed health inequalities appear to be mediated by both material deprivation and social fragmentation. Deprivation

\section{CLINICAL IMPLICATIONS}

- Area-level measures of social fragmentation have a strong influence on firstadmission rates for psychoses.

- The association between deprivation and admission rates to hospital holds for first-ever admission rates for psychosis.

- Deprivation alone may not best describe the ecological relationship for admission rates for psychosis, so that causation is necessary when using such measures to inform changes in healthcare provision and service development.

\section{LIMITATIONS}

- The study was hampered by the lack of a generally accepted measure of rurality.

Social fragmentation and deprivation indices may not measure material deprivation in rural areas as accurately as in urban areas.

- Causal inferences could not be generated owing to the cross-sectional study design.

JUDITH ALLARDYCE, MRCPsych, Section of Psychological Medicine, University of Glasgow;

HARPER GILMOUR, BSc, MSc; JACQUELINE ATKINSON, PhD, Section of Public Health and Health Policy, University of Glasgow; TRACEY RAPSON, BSc, MSc, JENNIFER BISHOP, BSc, Information and Statistics Division Scotland, Edinburgh; R. G. McCREADIE, FRCPsych, Department of Clinical Research, Crichton Royal Hospital, Dumfries, UK

Correspondence: Dr Judith Allardyce, Section of Psychological Medicine, Academic Centre, Gartnavel Royal Hospital, 1055 Great Western Road, Glasgow GI2 0XH, UK. E-mail: j.allardyce@clinmed.gla.ac.uk

(First received 25 February 2004, accepted 9 December 2004)

scores such as Carstairs indices are often used to measure health inequalities between areas, but our results suggest that this would not fully describe the ecological relationship and that other measures of societal influences should be explored if we wish to clarify and tackle this inequality.

We are unable to determine whether the area-based measures operate at the individual (compositional) or at the macroenvironmental (contextual level). Further studies with individual and area-based measures of social fragmentation and deprivation and the onset of psychosis would clarify the relative importance of the personal and area characteristics.

\section{REFERENCES}

Allardyce, J., Morrison, G., McCreadie, R. G., et al (2000) Schizophrenia is not disappearing in south-west Scotland. British Journal of Psychiatry, 177, 38-4I.

Allardyce, J., Boydell, J., van Os, J., et al (200I) Comparison of the incidence of schizophrenia in rural Dumfries and Galloway and urban Camberwell. British Journal of Psychiatry, I79, 335-339.
Boardman, A. P., Hodgson, R. E., Lewis, M., et al (1997) Social indicators and the prediction of psychiatric admission in different diagnostic groups. British Journal of Psychiatry, I7I, 457-462.

Carr-Hill, R. (1988) Revising the RAWP formula: Indexing Deprivation and Modelling Demand. Discussion Paper 41. York: Centre for Health Economics, York University.

Carstairs, V. \& Morris, R. (1991) Deprivation and Health in Scotland. Aberdeen: Aberdeen University Press.

Congdon, P. (1996) Suicide and para-suicide in London: a small area study. Urban Studies, 33, 137-158.

Court, B.V. \& Cheng, K. K. (1995) Pros and cons of standardised mortality ratios. Lancet, 346, 1432-1433.

Dauncey, K., Giggs, J., Baker, K., et al (1993)

Schizophrenia in Nottingham: lifelong residential mobility of a cohort. British Journal of Psychiatry, $\mathbf{1 6 3}$. 613-619.

Faris, R. E. \& Dunham, H.W. (1939) Mental Disorders in Urban Areas. Chicago, IL: University of Chicago Press.

Flannigan, C. B., Glover, G. R., Wing, J. K., et al (1994) Inner London collaborative audit of admissions in two health districts. III: Reasons for acute admission to psychiatric wards. British Journal of Psychiatry, $\mathbf{1 6 5}$, 750-759.

Hare, E. H. (1956) Mental illness and social conditions in Bristol. Journal of Mental Science, 102, 349-357. 
Harley, K. \& Jones, C. (1996) Quality of Scottish morbidity records (SMR) data. Health Bulletin, 54, $410-417$.

Harrison, J., Barrow, S. \& Creed, F. (1995) Social deprivation and psychiatric admission rates among different diagnostic groups. British Journal of Psychiatry, 167, $450-462$

Harvey, C. A., Pantelis, C., Taylor, J., et al (1996) The Camden schizophrenia surveys. High prevalence of schizophrenia in an inner London borough and its relationship to socio-demographic factors. British Journal of Psychiatry, 168, 4I8-426.

Jarman, B., Hirsch, S., White, P., et al (1992) Predicting psychiatric admission rates. BMJ, 304, ||46-||5|.

Kendrick, S. \& Clarke, J. (1993) The Scottish record linkage system. Health Bulletin, 5I, 72-79.

Koppel, S. \& McGuffin, P. (1999) Socio-economic factors that predict psychiatric admissions at a local level. Psychological Medicine, 29, |235-124|.
Lewis, G., David, A., Andreasson, S., et al (1992) Schizophrenia and city life. Lancet, 340, 137-140.

Marcelis, M., Navarro-Mateu, F., Murray, R. M., et al (1998) Urbanisation and psychosis: a study of 1942-1978 birth cohorts in the Netherlands. Psychological Medicine, 28, 87I-879.

McLoone, P. (1995) Carstairs Scores for Scottish Post Code Sectors in the 1991 Census. Glasgow: Public Health Research Unit.

Midwinter, A., Mair, C. \& Moxen, J. (1988) Rural Deprivation in Scotland. An Investigation into the Cases for a Rural Fund. London: HMSO.

Mortensen, P. B., Pedersen, G. B., Westergaard, T., et al (1999) Effects of family history and place and season of birth on the risk of schizophrenia. New England Journal of Medicine, 340, 603-608.

Reijneveld, S. A., Verheij, R. A. \& de Bakker, D. H. (2000) The impact of area deprivation on differences in health: does the choice of geographical classification matter? Journal of Epidemiology and Community Health, 54, 306-313.

Smith, G. D. \& Hart, C. (1999) The use of census based aggregate variables to proxy for socio-economic group: evidence from national samples. American Journal of Epidemiology, I50, 996-997.

Thornicroft, G., Bisoffi, G., De Salvia, D., et al (1993) Urban-rural differences in the associations between social deprivation and psychiatric service utilisation in schizophrenia and all diagnoses: a case-register study in Northern Italy. Psychological Medicine, 23, 487-496.

van Os, J., Driessen, G., Gunther, N., et al (2000) Neighbourhood variation in incidence of schizophrenia: evidence for person-environment interaction. British Journal of Psychiatry, 176, 243-248.

World Health Organization (1978) International Statistical Classification of Diseases and Related Health Problems (ICD-9). Geneva: WHO. 


\section{CHAPTER 5}

\section{Variation in the rates of psychosis by area level social characteristics: A systematic review}

Allardyce,J \& Boydell, J: 2006

The wider social Environment and schizophrenia.

Schizophrenia Bulletin, 32:592-598. 


\section{Review: The Wider Social Environment and Schizophrenia}

\author{
Judith Allardyce ${ }^{1,2}$ and Jane Boydell ${ }^{3}$ \\ ${ }^{2}$ Department of Clinical Research, Crichton Royal Hospital, \\ Dumfries DG1 4TG, Scotland; ${ }^{3}$ Divison of Psychological \\ Medicine, Institute of Psychiatry, Denmark Hill, London SE5 \\ $8 \mathrm{AF}, \mathrm{UK}$
}

Rates of schizophrenia differ significantly between groups defined at the social level, eg, urban/rural comparisons, neighborhoods, and ethnic minority status. While earlier studies were not able to determine if the social environment influenced the development of schizophrenia (causation) or whether individuals at risk aggregated in adverse social environments (selection), the recent development of multilevel modeling should inform this debate. To date, there are few examples of multilevel analyses in schizophrenia research; however, the small number of studies suggest that there may be a neighborhood social contextual effect that influences rates of schizophrenia and other psychotic disorders.

Further research is urgently required to progress our knowledge of how individuals, their genes, and the neighborhoods they live in, interact with each other. Studies need to use well-specified multilevel models, and until then, we should remain cautious in our interpretation of such findings.

Key words: schizophrenia/psychosis/multi-level/ ecological/deprivation/social cohesion/ethnic minority status

\section{Introduction}

The last decade has witnessed a shift from individualistic approaches in chronic disease epidemiology to the exploration of a wider set of risk factors, defined at levels higher (eg, families, neighborhoods, countries, or cultural context) and lower (eg, genetic or molecular) than the person. With this has come a rapid growth in studies examining the influence of the shared social environment on health. ${ }^{1,2}$

\footnotetext{
${ }^{1}$ To whom correspondence should beaddressed; tel: 44-1387-244000, fax: 44-1387-257735, e-mail: j.allardyce@clinmed.gla.ac.uk.
}

The study of schizophrenia has only recently started to reflect this methodological transition, due to the accretion of evidence showing consistently higher incidence rates for schizophrenia in urban as compared with rural areas with a dose-response relationship, possibly pointing toward a social causation rather than a social drift explanation for the observed urban-rural gradient. ${ }^{3}$ However, growing up in an urban area is a proxy environmental risk factor, and the correlates of this urban exposure, acting to increase the risk of schizophrenia, require elucidation. At an ecological level a neighborhood's social and economic structure may be an important explanatory factor for this urban effect. ${ }^{4}$

The social environment varies widely and systematically across neighborhoods, along the dimensions of deprivation, residential stability (eg, tenured housing and migration), family structure (eg, living alone), and ethnic composition. ${ }^{5}$ Social stratification by place is inherent and pervasive, ${ }^{6,7}$ and many health outcomes vary across them. ${ }^{8}$ This ecological perspective is not new in schizophrenic research; as far back as 1939, Faris and Dunham ${ }^{9}$ demonstrated differential rates of schizophrenia across city zones in Chicago, with the highest rates in areas characterized by high ethnic conflict, residential mobility, and social disorganization. Later studies from Bristol ${ }^{10}$ and Nottingham $^{11}$ demonstrated similar associations. However, we abandoned ecological studies concentrating our effort on individual approaches in the 1970s; with the demonstration that schizophrenia was a biological brain disorder, we thought that its causes, effective treatments, and prevention strategies would also be biological and lie at the individual level and assumed that differential rates across neighborhoods were due to social (selection) drift. ${ }^{12}$

In this review, we describe the current state of published research examining neighborhood-level social environmental factors and schizophrenia. Studies were included (see Appendix) if (1) they were published after 1995, a period where multilevel modeling were established; (2) the area measured was the "neighborhood" or geographically defined small area; (3) outcomes were rates of psychosis or schizophrenia; and (4) there was an area-level measure of social context. Only 13 studies fulfilled these criteria. Deconstructing a neighborhood's social environment in a way that is susceptible to scientific inquiry is difficult; we have chosen to organize this review around 
the classical social area dimensions (stratifications) seen in neighborhoods such as (1) deprivation; (2) community organizational structure, ie, family composition and housing characteristics which support stable neighborhoods; and (3) ethnic composition.

\section{Area-Level Measures of the Social Environment and Their Relationship to Rates of Schizophrenia}

\section{Deprivation}

Ecological studies have consistently found a relationship (not necessarily linear) between derivation and incidence of psychosis ${ }^{13}$ first admission rates for schizophrenia, ${ }^{4,14}$ prevalence of schizophrenia, ${ }^{15,16}$ and admission rates for schizophrenia. ${ }^{17-20}$ The majority of these studies have used only aggregate-level data, reflecting their primary aim of assessing whether area measures of deprivation (used to determine formulas for national funding) can effectively predict use of mental health services. They describe the association but do not explain it. They do not differentiate between "compositional" effects (aggregations of persons each with increased individual risk) and "contextual" explanations where the features of the social (or physical) environment of the neighborhood influence the health of those exposed to it (either in addition to or in interaction with individual risk factors).

With the recent development of multilevel (hierarchical) statistical modeling, it is now possible to tease out the effects of individual-level risk factors and neighborhood contextual effects. The first study to examine both individual and neighborhood deprivation data ${ }^{15}$ on prevalence rates of schizophrenia showed that both individual characteristics and area-level deprivation were independently and significantly related to rates of schizophrenia. However, further analyses of the same data set ${ }^{16}$ including a specific measure of individual-level deprivation (household income) attenuated and rendered the neighborhood deprivation effect nonsignificant. The attenuation was due solely to individual deprivation indicators. Further studies have shown a similar attenuation in the neighborhood effect of deprivation after adjustment of individual-level risk factors (and other neighborhood measures). ${ }^{21,22}$

"Controlling away" the neighborhood deprivation effect could be (1) individuals predisposed to schizophrenia aggregating in deprived areas; (2) it may be that an individual's deprivation status is a function of neighborhood social characteristic not adequately captured by deprivation indices, eg, social disorganization; and (3) methodological artifact, area deprivation, is not conceptualized independently of the individuals living in the area. ${ }^{23}$ This may be overcome by using income/deprivation distribution or inequality measures rather than deprivation indices. $^{24}$ One study has examined the relationship of inequality and schizophrenia. ${ }^{25}$ They found that (social) inequality was not associated with higher incidence rates for schizophrenia except in the most deprived electoral wards, after controlling for individual ethnic minority status and area derivation. However, they did not measure individual social economic status, so it may be that, in the deprived/high inequality areas where there is likely to be very high concentrations (as compared with areas with less disparity) of deprived people, the contextual effect may have been overestimated.

\section{Neighborhood Organization (levels of disorganization)}

To date, most studies have used objective measurements of disorganization, generated from single or composite (Social Fragmentation index [SFI]) aggregates of the census variables-unmarried, 1-person household, population turnover, and private rents.

Two studies have shown that area measures of SFI have a strong influence on rates of psychosis ${ }^{4}$ and schizophrenia $^{18}$ independent of area-level deprivation and ethnic composition. Another cross-sectional study from Sweden used subjective survey methods and found higher rates of schizophrenia in areas characterized by high levels of disorder, fear of crime, and victimization. ${ }^{26}$ These studies had no individual-level data and, therefore, could not examine whether this is a contextual effect of the neighborhood. There are 2 published studies using multilevel analyses to explore organizational structure of neighborhoods. In Maastricht, single people were at greatest risk of schizophrenia in neighborhoods with smaller proportions of single people, ie, individual risk was conditioned on the neighborhood organization. ${ }^{22}$ The second study from the United States ${ }^{16}$ found residential mobility (population turnover and rented accommodation)-predicted prevalence of schizophrenia independent of area deprivation, ethnic composition, and individual social economic status. It is possible that important individual risk factors, eg, family histories, which were not controlled for, confound these results. Finally, we cannot assess the direction of this association, social disorganization might increase the risk of schizophrenia or possibly the social behavior of people with schizophrenia may increase the social disorganization in the areas where they live.

\section{Ethnic Composition (minority status)}

Again, we find a strong ecological relationship between proportion of people from an ethnic minority in an area and its rate of service use $\mathrm{s}^{16,18,21}$ which is attenuated when individual-level ethnicity is adjusted for. ${ }^{16,21}$ When people with a particular characteristic live in an area where this characteristic is less common, we observe higher rate of mental illness. ${ }^{27}$ Boydell et al ${ }^{21}$ examined this with regard to ethnicity and found incidence rates of schizophrenia to increase in ethnic minority groups as the proportion of ethnic minorities in the locality fell; this association has recently been replicated (not yet published) ${ }^{28}$ Further work is required, including measures of individual-level social disadvantage. It may be interesting 
to measure how minority groups are integrated/segregated in a neighborhood, along with subjective measures of how they perceive their environment, to clarify what aspect of the social experience (eg, discrimination) increases risk.

\section{Is There Evidence of Contextual Neighborhood Effect?}

Multilevel modeling is a recent advancement, but the initial findings are promising and suggest that high rates of psychosis in the most socially disorganized areas (rather than the most deprived areas) are contextual neighborhood effects. Individual risks of psychosis for minorities' groups seem to be conditioned on neighborhood ethnic density. These findings need to be replicated and study designs developed to extend the hypothesis of a neighborhood contextual influence.

Social cohesion may be the concept, which bridges these 2 neighborhood effects. Both may reflect populations that are generally less likely to form stable neighborhood ties, residential mobility impeding bonding in disorganized area, while people from minority groups may be more vulnerable to discrimination, perceived alienation, and anomie when they are a smaller minority. A socially cohesive area has richly endowed stocks of social capital (features such as levels of interpersonal trust, norms of reciprocity, and mutual aid, which act as resources for individuals and facilitate collective action). ${ }^{29,30}$ Social capital may be a protective factor for development of schizophrenia, though the potential mechanisms for this effect need to be developed. Social capital may work as a "buffer" modulating stress and dopamine regulation, mechanisms in the development of psychosis, ${ }^{31}$ or through psychological mechanisms; areas of low social capital may promote development of persecutory attribution styles, which in people with a genetic liability, may eventually manifest as a fully blown psychotic episode. ${ }^{32}$ Further research using more sophisticated measures of the social environment ${ }^{33,34}$ examining its effect across the life course, at different aggregation levels, and using well-specified multilevel models should help elucidate the influence of the wider social environment on schizophrenia.

\section{Appendix}

\section{Search Strategy}

The studies included in this review were identified by keyword searches MEDLINE, EMBASE, PsychINFO, and Science and the Social Science Citation Index using combinations of the keywords "schizophrenia" OR "psychosis" AND "neighbourhood," "ecological," "multi-level”, "hierarchical", "socioeconomic factor" "socioeconomic deprivation" "deprivation" "material deprivation", "social disadvantage" "inequality" "poverty" "social cohesion" "social disorganisation", "social fragmentation", "social capital" "residential mobility" "ethnicity".

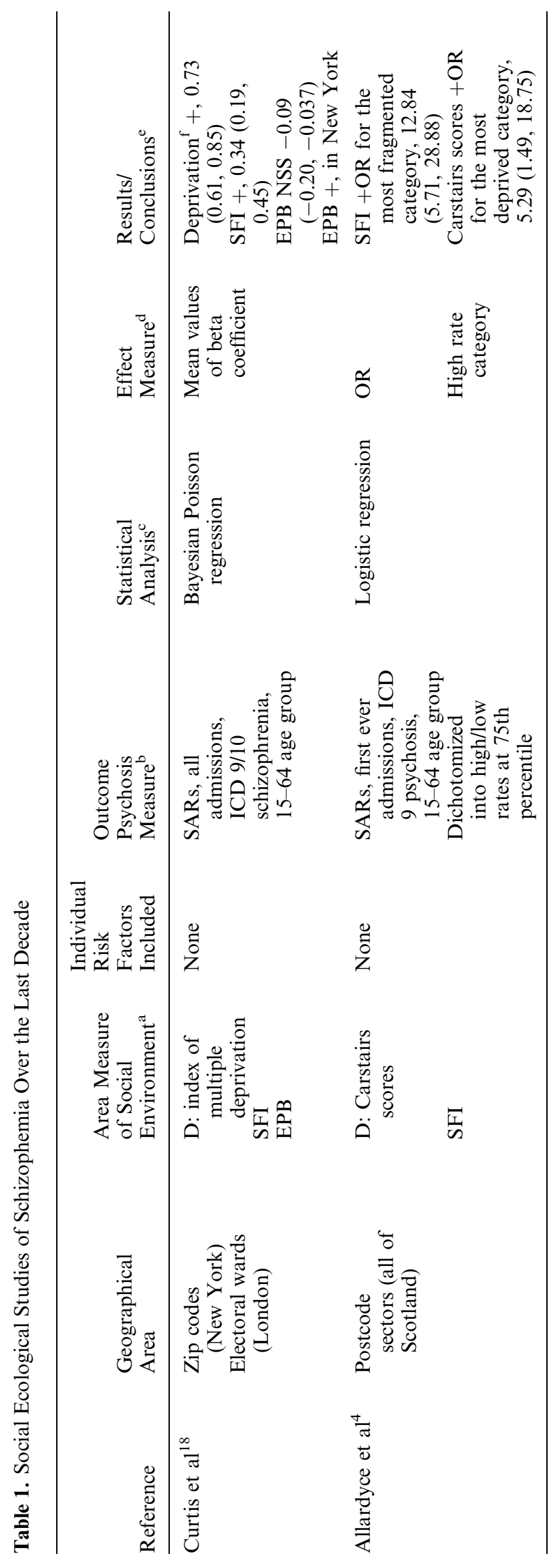




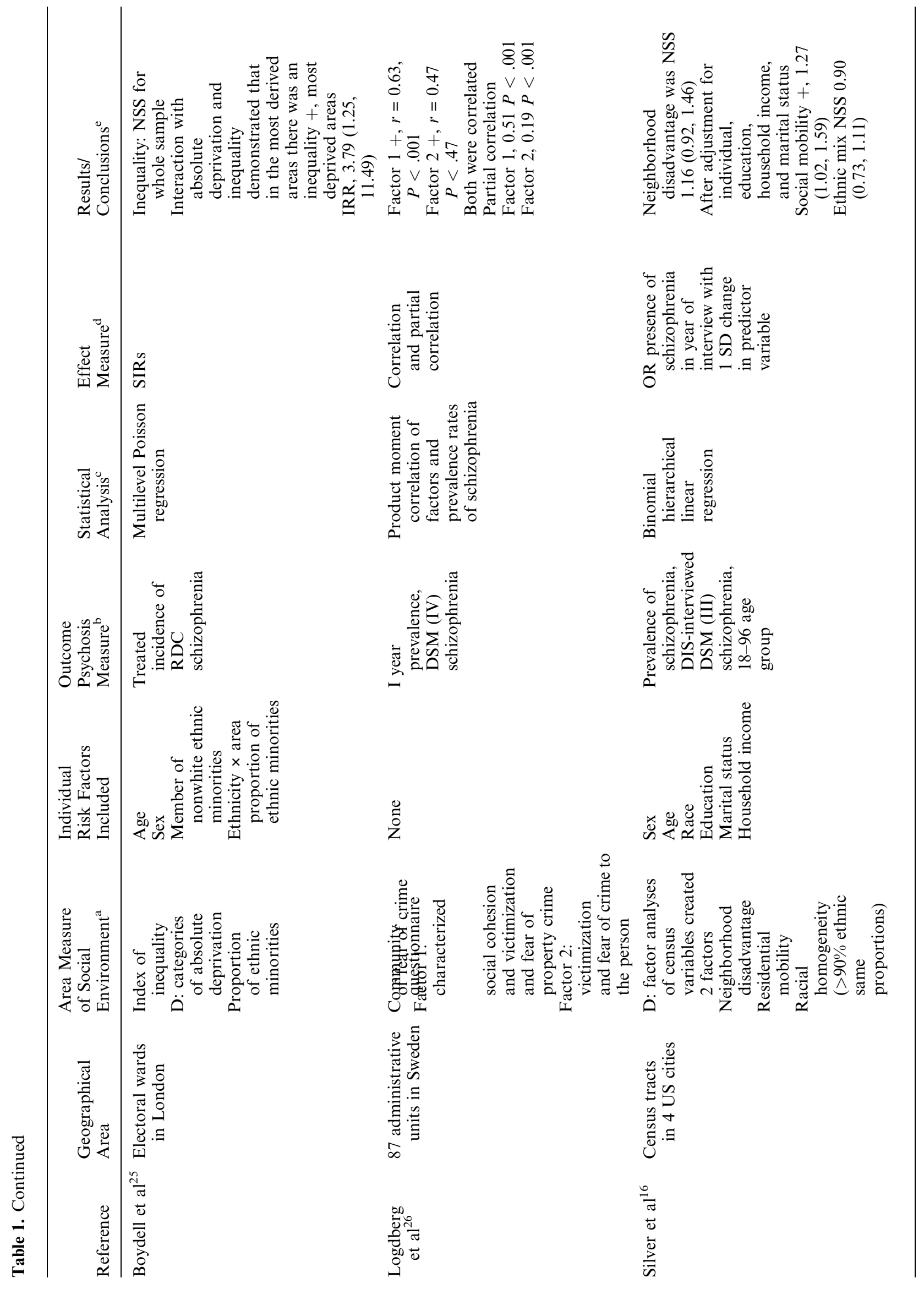




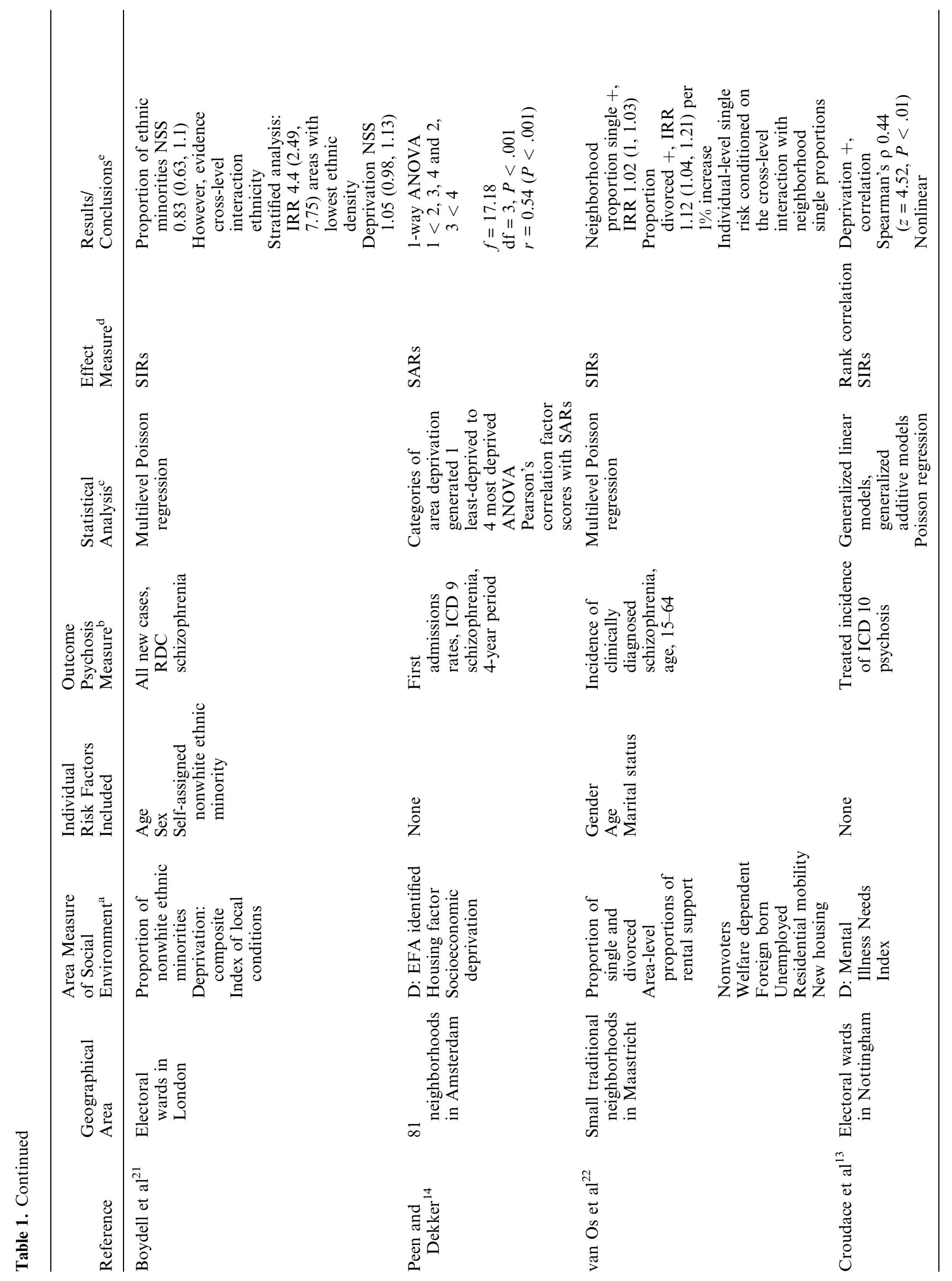




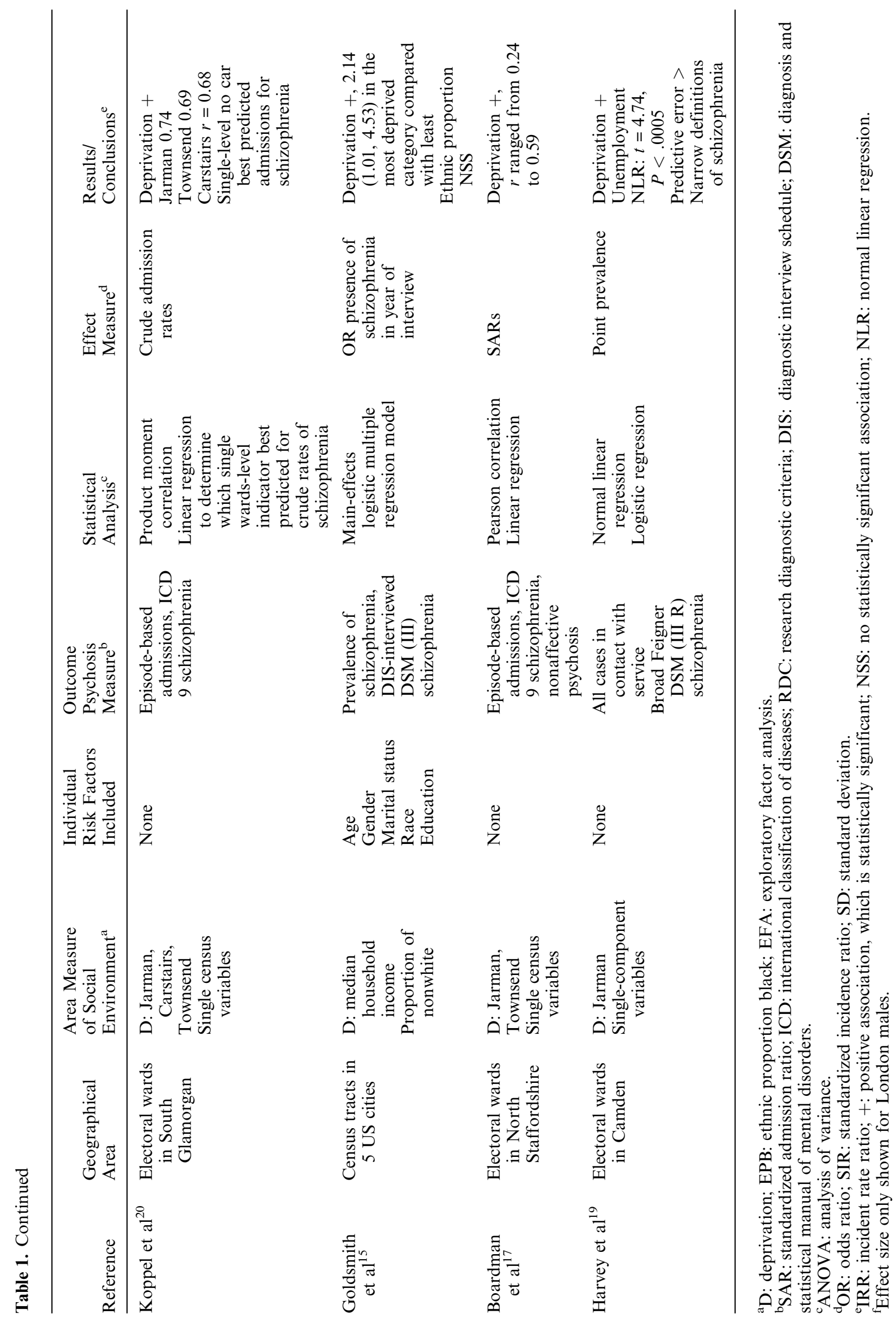




\section{References}

1. Berkman LF, Kawachi I, eds. Social Epidemiology. New York: Oxford University Press; 2000.

2. Oakes JM, Kaufman JS, eds. Methods in Social Epidemiology. San Francisco, Calif: John Wiley \& Sons; 2006.

3. Krabbendam L, van Os J. Schizophrenia and urbanicity: a major environmental influence - conditional on genetic risk. Schizophr Bull. 2005;31:795-799.

4. Allardyce J, Gilmour H, Atkinson J, Rapson T, Bishop J, McCreadie RG. Social fragmentation, deprivation and urbanicity: relation to first-admission rates for psychoses. Br J Psychiatry. 2005;187:401-406.

5. Sampson RJ. The neighborhood context of well-being. Perspect Biol Med. 2003;46(3 suppl):S53-S64.

6. Jason CG. A preliminary report in Swedish urban spatial structure. Econ Geogr. 1971;47(suppl 2):249-257.

7. Massey DS. The age of extremes: concentrated affluence and poverty in the twenty first century. Demography. 1996;33:395-412.

8. Marmot M, Wilkinson RG, eds. Social Determinants of Health. Oxford, England: Oxford University Press; 1999.

9. Faris REL, Dunham HW. Mental Disorders in Urban Areas. Chicago, Ill: University of Chicago Press; 1939.

10. Hare EH. Mental illness and social conditions in Bristol. J Ment Sci. 1956;102:349-357.

11. Giggs JA. Mental disorders and the ecological structure in Nottingham. Soc Sci Med. 1970;22:945-961.

12. Dohrenwend BP, Levav I, Shrout PE, et al. Socioeconomic status psychiatric disorders and causation-selection issue. Science. 1992;255:946-952.

13. Croudace TJ, Kayne R, Jones PB, Harrison GL. Non-linear relationship between an index of social deprivation, psychiatric admission prevalence and the incidence of psychosis. Psychol Med. 2000;30:177-185.

14. Peen J, Dekker J. Social deprivation and psychiatric service use for different diagnostic groups. Soc Sci Med. 2001;53: $1-8$.

15. Goldsmith HF, Holzer CE III, Manderscheid RW. Neighborhood characteristics and mental illness. Eval Program Plann. 1998;21:211-225.

16. Silver E, Mulvey EP, Swanson JW. Neighborhood structural characteristics and mental disorder: Faris and Dunham revisited. Soc Sci Med. 2002;55:1457-1470.

17. Boardman AP, Hodgson RE, Lewis M, Allen K. Social indicators and the prediction of psychiatric admission in different diagnostic groups. $B r \quad J$ Psychiatry. 1997;171: 457-462.

18. Curtis S, Copeland A, Fagg J, Congdon P, Almog M, Fitzpatrick $\mathrm{J}$. The ecological relationship between deprivation, social isolation and rates of hospital admission for acute psychiatric care: a comparison of London and New York City. Health Place. 2006;12:19-37.

19. Harvey CA, Pantelis C, Taylor J, et al. The Camden schizophrenia surveys. II. High prevalence of schizophrenia in an inner London borough and its relationship to socio-demographic factors. Br J Psychiatry. 1996;168:418-426.

20. Koppel S, McGuffin P. Socio-economic factors that predict psychiatric admissions at a local level. Psychol Med. 1999;29:1235-1241.

21. Boydell J, van Os J, McKenzie K, et al. Incidence of schizophrenia in ethnic minorities in London: ecological study into interactions with environment. BMJ. 2001;323:1336-1338.

22. van Os J, Driessen G, Gunther N, Delespaul P. Neighbourhood variation in incidence of schizophrenia. Evidence for person-environment interaction. $B r \quad J$ Psychiatry. 2000; 176:243-248.

23. Oakes JM. The (mis)estimation of neighbourhood effects: causal inference for a practicable epidemiology. Soc Sci Med. 2004;58:1929-1952.

24. Wilkinson RG. Unhealthy Societies: The Affliction of Inequality. London: Routledge; 1996.

25. Boydell J, van Os J, McKenzie K, Murray RM. The association of inequality with the incidence of schizophrenia - an ecological study. Soc Psychiatry Psychiatr Epidemiol. 2004;39:597-599.

26. Logdberg B, Nilsson LL, Levander MT, Levander S. Schizophrenia, neighbourhood, and crime. Acta Psychiatr Scand. 2004;110:92-97.

27. Wechsler H, Pugh TF. Fit of individual and community characteristics and rates of psychiatric hospitalisation. Am J Sociol. 1967;73:331-338.

28. Veling WA, Selten JP, Van Hoeken D, Hoek HW, Mackenbach JP. Ethnic density and incidence of schizophrenia in ethnic minorities in the Netherlands [abstract]. Schizoph Res. 2006;81:174.

29. Coleman JS. Foundations of Social Theory. Cambridge, Mass: Harvard University press; 1990.

30. Putnam RD. Making Democracy Work: Civic Traditions in Modern Italy. Princeton, NJ: Princeton University press; 1993.

31. Howes OD, McDonald C, Cannon M, Boydell J, Murray RM. Pathways to schizophrenia: the impact of environmental factors. Int J Neuropsychopharmacol. 2004;7(suppl 1):7-13.

32. van Os J, Hanssen M, Bijl RV, Vollebergh W. Prevalence of psychotic disorder and community level of psychosis symptoms: an urban-rural comparison. Arch Gen Psychiatry. 2001;58:663-668.

33. McCulloch A. Social environments and health: cross sectional national survey. BMJ. 2001;323:208-209.

34. Sampson RJ, Raudenbush SW, Earls F. Neighborhoods and violent crime: a multilevel study of collective efficacy. Science. 1997;277:918-924. 



\section{Part 2}




\section{CHAPTER 6}

\section{Variation onset of schizophrenia by person}

van Os J \& Allardyce J 2009

Presented are extracts from: The Clinical Epidemiology of Schizophrenia, in Kaplan \& Sadock's Comprehensive Textbook of Psychiatry (Ninth Edition) Lippincott \& Wilkins Publications: estimated publication date November 2009. 


\section{RISK FACTORS FOR THE ONSET OF SCHIZOPHRENIA}

\section{Genetic Risk: Indirect evidence from epidemiological studies ${ }^{(1 ; 2)}$}

Family studies compare the morbid risk (the proportion of affected to unaffected relatives, usually adjusted for variation in non-affected participants time-at-risk) for probands with schizophrenia, to the morbid risk in the general population or demographically matched control groups. Results from a systematic review of the studies published during the period 1980-2003, found the first-degree relatives of probands to have a higher morbidity risk for schizophrenia as compared to the relatives of controls. Estimates ranged from 2-9\% (in studies which adjusting for time at risk using Weinberg, Strömgren or Kaplan Meier methods) compared to a morbid risk in the relatives of controls of about $0.5 \%$. The effect varied according to the diagnostic classification employed in the study, broader definitions of schizophrenia, such as Research Diagnostic Criteria, tending to show larger estimated effects than the narrower definitions employed post DSM III and studies using control groups unscreened for psychiatric disorder showed more conservative estimates. There was also evidence of clinical heterogeneity, with the relatives of earlyonset cases having a higher morbid risk compared to the relatives of late onset cases. Further studies have examined familiar aggregation for categorical subtypes, symptom dimensions derived from factor analysis and individual symptoms. The findings are inconsistent and show significant methodological heterogeneity; there is nevertheless some support for higher morbid risk for schizophrenia associated with negative symptoms and the disorganization dimension / hebephrenic subtypes. Most family studies have investigated morbid risk in first-degree relatives; the few studies of second and third degree relatives suggest an increase in risk with increasing relationship closeness, the structural form of which may not be linear.

Adoption studies: Most children inherit their genetic characteristics and early rearing environment from the same biological parents. Adoption provides an opportunity to disentangle these genetic and rearing effects. Evidence from adoption studies have primarily come from 1) An adoptee's family study using the Danish national linked data set, comparing rates of schizophrenia in the adoptive and biological families of adoptees with schizophrenia. Results reported over the last 30 years (using different diagnostic criteria) consistently show the biological relatives to be at a higher risk of schizophrenia than the adoptive relatives, who have a similar estimated risk to the rest of the general population. 2) The second strategy compares the rates of schizophrenia in adopted away children, for parents with and without a diagnosis of schizophrenia. Early studies using this methodological approach consistently demonstrated higher rates of schizophrenia in the adopted away offspring of affected mothers, compared to the rates in the offspring of the unaffected controls. An ongoing Finnish study has improved on these earlier methodologies by identifying larger samples, using standardized interviews with home assessments and DSM operationalised diagnostic criteria. Its results support the early findings, showing a morbidity risk in the adopted away offspring of mothers with schizophrenia of $5.1 \%$, as compared with $1.6 \%$ in the adopted away offspring of control mothers.

Twin studies: examine the concordance rates for monozygotic (identical) twins (assumed to inherit all their genes in common) with those of dizygotic (non-identical) twins (who on average share around $50 \%$ of their genes). Such studies further assume that twin pairs are equally exposed to environmental risk factors for schizophrenia. Statistical combination of the published studies that used DSM IIIR criteria to define caseness, estimated pooled concordance rates of $50 \%$ in monozygotic twins and $4.1 \%$ in the dizygotic pairs, the pooled estimates of concordance for studies using different diagnostic classification systems are actually very similar. The con- 
cordance rate for monozygotic twins was higher in twins with an early rather than a late onset of schizophrenia. Twin studies investigating classical schizophrenia subtypes show higher monozygotic concordance rates for the hebephrenic subtype compared to the paranoid type. Combination twin and family studies find the risk for schizophrenia in the offspring of the identical and unaffected twin to be the same as that seen for the offspring of the affected twin.

\section{Specificity of Heritability}

Relationship to subclinical-psychoses phenotypes: Clinicians have long recognized that some close relatives of patients with schizophrenia, though never frankly psychotic, display symptoms and signs similar to the pathological experiences seen in the clinical disorder. Some are attenuated forms of the positive/disorganized symptoms of schizophrenia (odd behavior, odd speech, muddled thinking, loose associations, ideas of reference, magical thinking, suspiciousness and illusions) while others resemble the negative symptoms (social isolation, aloofness, guardedness poor rapport). The DSM classification system has combined these quasi-psychotic (schizotypic) features under the diagnostic category; schizotypal personality disorder. As schizotypal, schizoid, and paranoid personality disorders are found to co-occur they have been grouped together under the rubric Cluster-A personality Disorder within the manual. Family studies measuring lifetime prevalence for DSM cluster-A personality disorders (some studies have also included avoidant personality disorder) in first-degree relatives of patients with schizophrenia (and other psychotic disorders including psychotic bipolar disorder) find rates in the relatives of the probands (range: lifetime prevalence $2.8 \%-8.2 \%$ ) that are $2-4$ times the rates found in the control families. The significant heterogeneity in estimated lifetime prevalence is primarily due to methodological differences. Adoption and twin studies confirm this familiar aggregation. Negative-schizotypic features (and associated neuro-cognitive impairments) are more prevalent than positive schizotypic features in the relatives of patients with schizophrenia. Further, studies investigating the correlation of (factor analytically derived) 'pathological' symptom dimensions (positive negative and disorganized) in patients with their corresponding (factor analytic derived) non-pathological' schizotypal dimensions in the clinically well relatives, have consistently shown correlation of the negative 'pathological' dimension and negative schizotypal dimension, suggesting a discrete genetic liability effect across 'pathological' and 'non-pathological' negative symptoms. Less clear is the association of 'pathological' disorganized and positive symptom dimensions and their corresponding schizotypic dimensions. The familiar co-aggregation of 'pathological' and attenuated schizotypal symptoms suggests that their may be a single continuum of liability across the schizotypic features and 'pathological' psychotic symptoms.

Relationship to other psychotic disorders \& bi-polar disorder: Family and adoption studies of schizophrenia have generally shown an excess of risk in biological relatives, not only for schizophrenia, but also for a broad range of psychotic disorders, such as schizophreniform, schizoaffective, delusional, atypical, delusional disorders. Less accepted is the possible familiar coaggregation of bipolar disorder and schizophrenia in families, stemming primarily from the limited and conflicting data available from family studies of bipolar disorder, which have been interpreted to support the disorder 'breeding true'. However, schizoaffective disorder occurs at similarly increased rates in the families of probands with schizophrenia and bipolar disorder, and family studies of schizoaffective disorder demonstrate increased risk for both schizophrenia and bipolar disorder, suggesting at least some genetic non-independence. These studies do not help us elucidate the nature of this shared liability. A possible explanation would be an underlying continuous common liability for all psychotic disorders. A twin study from Finland tested the hypotheses that affective disorders and other psychotic disorders represented an intermediate threshold on a liability continuum shared with schizophrenia. The results did not support a sin- 
gle continuum liability model, though the investigators did not compare alternative hybrid models. However, a further study investigated both common liability (shared genetic effects) and disorder specific liability models, using the Maudsley twin data set. The researchers relaxed the normal hierarchical diagnostic approach, so allowing within person co-morbidity of schizophrenia schizoaffective and manic syndromes. Independent and common pathway models were used to compare the associations of the syndrome pairs and found that for schizophrenia and mania, the model encompassing both shared (common) and syndrome-specific genetic effects fitted the data best, while for schizoaffective disorder the shared genetic effect model was sufficient to explain the associations. These results suggest that transmission may operate through a set of susceptibility genes specific to schizophrenia, and another set specific to bipolar disorder, while a third set of shared (common) effects acts across the three syndromes. Interpretation of these studies must be tentative; findings are preliminary and somewhat crude (based on additive and static models of genetic liability) and further replication is required. These codicils not withstanding, the evidence point towards a model of liability, which incorporates a common (shared) genetic susceptibility for psychosis, and concomitant separate schizophrenia-specific liability. There have been substantially fewer studies examining the risk of non-affective and nonpsychotic Axis 1 disorders in the relatives of probands with schizophrenia; the current weight of evidence however suggests little or no familiar association.

\section{Genetic Model of Liability for Schizophrenia (3)}

The family, adoption and twin studies discussed above provide irrevocable evidence for the involvement of a major genetic component in the liability for schizophrenia. However, the familiar distributions are inconsistent with simple Mendelian modes of inheritance (single major locus) and are more compatible with a oligo/polygenetic mode of inheritance. One possible model is the polygenetic threshold model, which assumes the risk of schizophrenia is due to additive effects of multiple small genes, at different loci. However, the polygenetic threshold model does not adequately account for the exponential rise in risk of schizophrenia observed with increasing familiar closeness, which is more consistent with (at least some) epistasis (an interactive effect between 2 or more genes, such as suppression or alteration of the expression of one gene by another locus). Nonetheless, the monozygotic/dizygotic twin correlations are significantly lower than would be expected with a full epistatic model. Therefore, it is more likely that both genetic and environmental factors contribute to the development of schizophrenia. The mechanisms by which these factors interact to influence risk remains unclear, but will probably involve the complex interplay of both additive and interacting genes, environmental factors and geneenvironment interactions (genetic moderation of environmental factors and environmental moderation of gene expression), which are continuously distributed within the population. Further, this model of risk may vary across individuals, families or clinically defined subgroups of the disease, depending on the specific risk factors active within the person at different stages of development. If we accept this probabilistic model of risk transmission then environmental risk factors appear to be necessary complementary components in the development of schizophrenia, in many if not all cases.

\section{Gene-environment interaction (4;5)}

Traditional epidemiological risk factor methodologies measure the linear association of environmental exposures to risk for onset and persistence of schizophrenia. Such studies (discussed below) demonstrate that people respond differently to the same environmental exposure. Some individuals seem to have a low risk of developing the disorder as a result of an environmental 
exposure, while others are more vulnerable, or in some circumstances person's with increased liability for a disorder seem to have a higher exposure frequency to noxious environmental agents, as compared with the general population. Variation in genotype may in part, account for this heterogeneity, two of the most often studied gene-environment relationships are 1) Geneenvironment correlation: A genotype may increase the likelihood of a person becoming exposed to an environmental risk factor. For example, biological parents not only determine a child's genotype but also provide their early rearing environment; or a child's temperament (at least partially genetically determined) influences how he or she selects and/or shapes their environment. 2) Gene-environment interaction (synergism): Posits that instead of inheriting a disease state per se, a person actually inherits a set of genetic susceptibility factors, which act to moderate the effects of (causal) environmental exposures. If this assumption is correct, integrated epidemiological approaches, which examine the joint influence of genes and environment on the risk for schizophrenia, will improve the accuracy and precision of the measured risk estimates and the predictive power of environmental risk factors.

\section{ENVIRONMENTAL RISK FACTORS FOR THE ONSET OF SCHIZOPHRENIA}

A number of environmental factors are statistically associated with an increased risk for onset of schizophrenia. Evidence of association however, does not equate to causality. Decisions regarding causation require deductive judgments. In order to aid this logic, causal criteria have been developed (6) (Textbox 1). This chapter uses the most frequently applied criteria to structure the review of the current evidence for the risk factors for the onset of schizophrenia, however it is important to stress that these criteria should not be used in a mechanistic fashion, as all have exceptions and are useful at best as a framework for thought.

\section{RISK FACTORS OPERATING DURING “EARLY DEVELOPMENT”}

\section{Paternal Age}

Recently, several large population based cohort studies from Israel, USA, Denmark and Sweden have provided strong evidence linking advanced paternal age to the risk of schizophrenia in the offspring. Strength of the association: The association is strong, independent and the findings consistent. There is a higher risk of schizophrenia (around 3-4 times) in the offspring of fathers who are older than fifty, at the time of conception, compared to the offspring of fathers in their early twenties. The small variation of estimated effect size across studies reflects methodological differences, with higher associations found in studies of patients with narrowly defined schizophrenia. Dose-Response Relationship: Nearly all studies have found a dose-response relationship, with the risk increasing across consecutive age-bands. The functional form of this association has not established but there is some evidence for a slightly increased risk of schizophrenia in younger fathers (under 20) with J-shaped associations having been suggested. Temporal Association: exposure clearly predates diagnosis of schizophrenia. Consistency of findings \& potential sources of confounding and bias: All the cohort studies have estimated broadly similar strengths of association and while adjustment for known risk factors have been made, there may be residual confounding by a factor currently unidentified, which directly increases the risk of 


\section{TEXTBOX 1: CAUSAL CRITERIA}

\section{Strength of the association}

The argument states that strong associations are more likely to be causal, because if they could be explained by another factor the effect of the confounder would have to be even stronger than the observed association. While weaker associations are more likely due to 'noise' and bias It is important to remember that measures of association (eg relative risk for odds ratios) are a characteristic of the sample under study and depends on the relative prevalence of other causal factors.

\section{Dose-response relationship (biological gradient)}

Defines a unidirectional trend in disease frequency with increasing levels of exposure; however, this is neither necessary or sufficient for causality as the presence of an unadjusted confounder will also show a gradient. Further, some causal relationships show single jumps (thresholds).

\section{Temporal association}

This is a sine qua non for causality. Requires that for a factor to be considered causal it must precede the disease, this is irrefutable, however it does not provide evidence for or against causation when the cause is present prior to onset of the disorder.

\section{Consistency of findings}

Is the repeated observation of an association in different populations under different circumstances. However, lack of consistency does not rule out a causal association because some risk factors only produce effects under certain situations where a complement of causes are present (or have been present). Consistency can only really be assessed after the relevant details of the causal mechanism are understood.

\section{Specificity of effect}

This is still emphasised in psychiatric epidemiology, perhaps due to the continual nosological debates regarding classification of disorders. However, there are no good grounds to refute a causal interpretation of an exposure because it is related to multiple effects or disease outcomes.

\section{Biological plausibility}

Plausibility is important however, biologically plausible hypotheses can be constructed which are later found to be erroneous and vice versa causal associations can be dismissed due to current knowledge or belief systems.

\section{Coherence}

Related to biological plausibility and therefore similar problems, the association should not conflict with what is known of the natural history and biology of the disease.

\section{Experimental evidence}

Commentators are unclear what is meant by experimental evidence, wither it was evidence coming from laboratory work or human experiments such as removal of an exposure. However this is a test for causality rather than a criterion.

\section{Analogy}

Analogy may provide a source for more elaborate hypotheses about the associations but does not falsify a hypothesis.

\section{Critique of the causal criterion}

With the exception of temporality none of the above situations can be viewed as a necessary or sufficient criteria for determining wither an observed association is causal. Causal inference cannot be deduced with certainty.

schizophrenia and is also associated with paternal age. Specificity of effect: The association is stronger for narrowly defined schizophrenia compared to non-affective psychosis supporting relative specificity for schizophrenia within the spectrum of psychosis. Association with age at onset: no evidence for an association between age of first admission and paternal age has been demonstrated. Evidence of Gene-environment interactions: There is a stronger association between paternal age and schizophrenia in people without a family history (7). This observed 
pattern of effect lends support for two genetic theories. The first and most likely is that advancing paternal age results in accumulation of de novo mutations in the germ cells of older fathers, or secondly advancing paternal age interferes with the DNA-methylation process of gene expression.

\section{SEASON OF BIRTH ${ }^{(8 ; 9)}$}

Excess winter birth in people who later develop schizophrenia is a robust epidemiological finding, at least in the Northern Hemisphere. It is likely to be a 'proxy' indicator for some seasonally fluctuating environmental factor. The most popular hypotheses relate to seasonal variation in exposure to inter-uterine viral infections around the time of birth, or variation in light, temperature/weather or external toxins. Strength of the association: There is a statistically significant but small effect associated with winter birth and later development of schizophrenia, a metaanalysis of Northern hemisphere studies, estimated a pooled odds ratio of 1.07 (95\% confidence interval 1.05-1.08). Dose-response Relationship: the effect size increases with latitude (though there are fewer studies from the lower latitudes of the Northern hemisphere in the pooled analyses) until about 50 degrees latitude, where it then starts to fall, suggesting a nonlinear inverted $U$ shape association, this may in part be explained by methodological heterogeneity across studies. Temporal Association: exposure clearly predates diagnosis of schizophrenia Consistency of findings \& potential sources of confounding and bias: The studies from Northern hemisphere countries consistently show excess winter births in people with schizophrenia, however the effect seems to be greater for those born in urban as compared to rural settings. A meta-analysis of studies carried out in the southern hemisphere did not find an effect for season of birth (pooled odds ratio 1.04, 95\% confidence interval 0.99-1.08). Further pooled analyses of individual patient data, found a significant association with deficit syndrome schizophrenia (a group defined by primary enduring negative symptoms) and summer birth thus the season of birth effect pattern seems to differ across groups of patients with different clinical characteristics. Specificity of effect: Studies examining season of birth association to other psychiatric conditions are small and their findings inconsistent. The largest and most robust study, carried out in Denmark did not find an association with season of birth and development of bipolar disorder suggests some specificity of effect for schizophrenia (spectrum) disorder as compared with bipolar disorder. Association with age at onset: Few studies have looked at age of onset in relation to seasonality of birth in people with schizophrenia, the largest study to date found higher rates of winter births in patients who developed schizophrenia later in life, most studies are underpowered to investigate this issue. Evidence of Gene-Environment Interactions: Little information is currently available. Indirect support comes from a recent study In Puerto Rico, of 25 families with at least two siblings who were diagnosed with schizophrenia, affected relatives were more likely to have been born in the winter months, as compared to their unaffected family members, suggesting further research on interaction between winter birth and underlying genetic liability may be productive.

\section{PREGNANCY AND BIRTH COMPLICATIONS (10;11)}

There are a large number of published studies demonstrating some relationship between pregnancy and birth complications and the development of schizophrenia. Strength of the association: The literature suggests pregnancy and birth complications have a small effect on the risk of 
later development of schizophrenia. Meta-analytic studies have estimated a pooled odds ratio of 2.0 (95\% confidence intervals 1.6-2.4) for patients with schizophrenia having a history of pregnancy and birth complications compared with non-affected controls. The term 'pregnancy and birth complications' covers a broad range of pre and peri-natal events but even a large systematic review of prospective population-based studies (period 1995-2001) was unable to identify one individual complication or unifying mechanism. The investigators found three main categories of obstetric complication to have significant estimates, i) abnormal fetal growth and development: low birth weight, congenital malformations and small head circumference. ii) Complications of pregnancy: bleeding, pre-eclampsia, diabetes and rhesus incompatibility. iii) Complications of delivery: asphyxia, uterine-atony and emergency cesarean section. Taken together, they seem to implicate an increased risk of hypoxia. Dose-response Relationship: Studies to date have mostly been underpowered to uncover variation in effect size (odds ratio) across groups ordered by degree of exposure to pregnancy and birth complications. One population basedcohort study from Helsinki used continuous measures and found an effect gradient of increasing risk with decreasing birth weight, length at birth and placental weight (controlled for family history of schizophrenia) and The National Collaborative Perinatal Project found risk increased when multiple hypoxia-associated complications were present. However, it is difficult to interpret these gradients while the underlying mechanism remains elusive. Temporal Association: exposure clearly predates diagnosis of schizophrenia, though fetal growth retardation may be an early indicator of aberrant neurodevelopment. Consistency of findings \& potential sources of confounding and bias: The associations for individual obstetric complication with later development of schizophrenia are mostly negative and inconsistent; however, statistically pooled estimations suggest a modest effect with the broad category of pregnancy and birth complications. There is methodological heterogeneity with a discrepancy in the estimated effects for case control studies (that generally show stronger associations) and cohort studies. The most likely explanation for this is selection and information bias, in the of case control studies. Pregnancy, birth and neonatal complications do not act independently of each other; however, current studies are underpowered to detect interactive effects. There have been few studies examining differential effect of pregnancy and birth complications and symptom dimensions or subtypes of schizophrenia. A small study from Poland found pregnancy and birth complications to be significantly more frequent in patients with prominent negative symptoms compared with patients with prominent positive symptoms. Specificity of effect: A recent systematic review of pregnancy and birth complications and bipolar disorder, estimated the pooled odds ratio for exposure to pregnancy and birth complications and later development of bipolar disorder to be 1.01 (95\% Confidence interval 0.76-1.35) compared to controls, 1.13 (95\% confidence interval 0.641.99) compared with patients with unipolar disorders and 0.61 (95\% confidence interval 0.39 0.95) compared with people who later developed schizophrenia. Furthermore, a Danish National Register based study did not find fetal growth indicators to be associated with the development of bipolar disorder. This suggests some specificity for schizophrenia (spectrum) disorder among psychiatric conditions. Association with age at onset: a meta-analytic study reanalyzed individual patient data from 11 case-control studies of adult onset schizophrenia, the pooled odds ratio was 1.52 (95\% confidence intervals 1.04-2.2) for patients with earlier age of onset (under 22) for broadly defined pregnancy and birth complications compared with the later onset group. Further analysis divided the age distribution into quartiles and found a gradient of association across age groups (adjusted for study design). However, two cohort studies of childhood onset schizophrenia have not found stronger effects in very early onset cases. Evidence of Geneenvironment interactions: Indirect evidence comes from a population based cohort study in Helsinki, which found that a history of fetal hypoxia was associated with increased structural brain abnormalities (reduced grey matter and increased ventricular size) among schizophrenia 
patients and their non-affected siblings, but not among controls at lower genetic risk for the disorder. Moreover, the effect size was greater for patients compared to the unaffected siblings, suggesting that while they show some sensitivity to the 'schizophrenia-promoting' effects of hypoxia there is a quantitative difference in this sensitivity. Gene-environment correlation: Evidence from a meta-analytic study of pregnancy and birth complications in children born to parents with schizophrenia found individuals with schizophrenia to have an increased risk of obstetric complications, low birth weight and poor neonatal condition. The risk is greater for mothers with schizophrenia but is not confined to mothers with onset prior to delivery or to the births of the children who develop schizophrenia themselves later in life. These findings possibly reflect a gene environment correlation where by the genetic liability for schizophrenia in the parent increases the likelihood of social adversity which is associated with obstetric complications.

\section{OTHER PUTATIVE PRENATAL RISK FACTORS}

Emerging evidence from ecological studies suggests that a number of other prenatal factors may have a weak association with the later development of schizophrenia Prenatal infections (12). A series of studies have found higher rates of schizophrenia in people exposed to the 1957 influenza pandemic during the second trimester of their gestation. This is not a fully replicated finding and remains controversial. A study from New York City found a 10-20\% increased risk for schizophrenia in people who had serologically confirmed prenatal rubella exposure, while further studies have implicated prenatal exposure to toxoplasmosis, polivirus and other common respiratory infections. The effect may be in part due to cytokines and chemokines, which mediate host response to infection. Rhesus Incompatibility There is some evidence that rhesus incompatibility increases the risk of developing schizophrenia; this may not be through the direct toxic effect of hyperbilirubinemia on the developing brain, but rather via maternal-fetal genotype incompatibility effects. Prenatal stress: There may be higher rates of schizophrenia in the offspring of mothers who experienced significant levels of stress during pregnancy, such as the death of a spouse, living through a natural disasters or military invasions. Famine during preg-

nancy: Nutritional deficiency in pregnancy may also increase the risk of schizophrenia in offspring. A two-fold increase in rates of schizophrenia was found in the children of mothers who were exposed to the Dutch famine, during early gestation. These putative environmental risk factors all require further replication.

\section{RISK FACTORS OPERATING DURING CHILDHOOD AND ADOLESCENCE}

\section{Urban birth \& upbringing $(13 ; 14)$}

Over twenty studies have demonstrated an association between urban living and rates of schizophrenia and non-affective psychosis. Strength of the association: Generally, studies show a two-fold increase in risk of schizophrenia in urban as compared to rural settings. Doseresponse Relationship: The weight of evidence supports a risk gradient, with unidirectional increasing rates of schizophrenia with increasing levels of urbanicity. Temporal Association: There is compelling evidence suggesting that elevated rates of schizophrenia in urban areas are not due to social drift during the prodrome (reverse causation). It is urban exposure prior to the onset of the disorder, which seems to be more important rather than the level of exposure at the 
time of illness onset. A case register study from The Netherlands demonstrated an increased risk of schizophrenia for people born in urban areas irrespective of where they were living at the time of presentation, while those living in urban areas were only at increased risk if they had also been born within an urban area. Furthermore, a Danish study measured the effect of cumulative urban exposure on risk of schizophrenia and found the first fifteen years of life were the most critical. Analyses from the same cohort further suggest that people conceived (including period of gestational development) in urban areas but reared in less urban areas may be at higher risk of schizophrenia (15). This phenomenon of increased risk seen when there is a mismatch between in-utero developmental environments versus environment in adult life is recognized in other chronic complex diseases, such as cardiovascular disease. Consistency of findings \& potential sources of confounding and bias: The findings are consistent with similar effect sizes seen across studies from different geographical areas. The effect is not confounded by other known risk such as pregnancy and birth complications and is present after controlling for socio-demographic variables. Specificity of effect: Most analyses have examined the association of urban living within the broad psychosis category rather than specific diagnoses. Studies, which have compared across diagnostic categories, have found the risk to be relatively specific for schizophrenia (spectrum) disorders compared to bipolar disorder. Association with age at onset: Few investigators have examined this, but those that have consistently find an earlier age of onset in people exposed to urban upbringing. Evidence of gene-environment interactions: Studies investigating family history and urban exposure support a possible interaction with genetic liability. For example in the Danish cohort, those without a family history of schizophrenia had a much smaller risk of schizophrenia ( $0.054 \%$ increase risk, per unit change of urbanicity) compared to those with a family history ( $0.22 \%$ increase in risk, per unit change of urbanicity).

\section{Could group level social processes account for the urban upbringing effect?}

Growing up in an urban environment is a proxy environmental risk factor. Recent research has started to examine the social and economic structure of neighborhoods as a possible explanation for this effect. The social environment varies widely and systematically across dimensions of material deprivation, neighborhood organization (social fragmentation) and ethnic composition. Deprivation: Studies have consistently found a relationship (not necessarily linear) between material deprivation and risk of schizophrenia, though study design has generally been cross sectional therefore the direction of the relationship can not be assessed, and could be accounted for by people drifting into more deprived areas before presenting to clinical services. Social Fragmentation/social cohesion: Ecological studies have consistently shown area level social fragmentation to influence strongly the rates of schizophrenia. A small number of recent studies have examined levels of social cohesion and found neighborhoods richly endowed with stocks of social capital (interpersonal trust, norms of reciprocity, mutual aid) to have reduced rates of psychosis independent of material deprivation. Ethnic density: is an area level characteristic, defined as the percent composition (in a particular geographical area) of people with a given ethnicity, and has been found to be protective. That is, the higher the area ethnic density the lower the rates of schizophrenia for the ethnic group under study, independent of area measures of deprivation. Taken together these reports suggest a possible neighborhood level social process influencing the risk of schizophrenia, which will require further elaboration and replication.

\section{MIGRATION (16)}

Many studies have reported increased rates of schizophrenia in migrants, especially among the second-generation born in the new homeland. Increased risk is found among African Caribbean 
and black African immigrants to the UK; Surinamese Dutch-Antillean and Moroccan immigrants to The Netherlands; Australian, African and Greenland immigrants to Denmark and Greek immigrants to Belgium. Strength of the association: A meta-analysis of incidence studies (published between 1970-2003) found a higher risk of schizophrenia in almost all immigrant groups compared with the majority population. Although the pooled effect size suggested a three-fold increase in risk, there was evidence of systematic heterogeneity across studies, which could not be accounted for by variation in study design. Analyzing first and second-generation migrants separately found a pooled relative risk of 2.7 (95\% confidence intervals 2.3-3.2) for the first generation and a higher pooled relative risk of 4.5 (95\% confidence intervals 1.5-13.1) for the second generation. There was also a higher risk of schizophrenia in migrants from developing countries, however the most conspicuous finding was the increased risk associated with being black, with a pooled relative risk of 4.8 (95\% confidence interval 3.7-6.2). Dose-response Relationship: migrant status is a binary exposure; one is either a migrant or one is not. Therefore, a doseresponse association will only be testable after the underlying mechanism operating to increase the risk is established. Temporal Association: The selective migration of people at increased risk of schizophrenia is possible (reverse causation). However, this does not explain the increased risk, apparent in groups who migrated on mass, such as the Surinamese mass migration to the Netherlands. Further, it cannot explain the increased risk found in the second-generation immigrants. Consistency of findings \& potential sources of confounding and bias: Increased risk of schizophrenia in migrant groups is a replicated and consistent finding. The validity of this finding has been intensely debated, however. Increased rates do not appear to be explained by psychiatrists diagnosing schizophrenia differently in migrant groups, differential service use or under-enumeration, which may have hampered some of the earlier studies. Poor obstetric care in migrant mothers has also been muted as an explanation but there is no evidence to support this view. The few published studies examining migrant status across dimensions or subtypes of schizophrenia have not detected any differential associations between migrant group and the majority population. Finally, studies mostly from the United Kingdom have found the risk of schizophrenia to vary across different ethnic groups. The African Caribbean and black African groups in England are at higher risk than the Asian group, as are the Surinamese and Moroccan groups compared to the Turkish group in the Netherlands. Specificity of effect: The association is not specific to schizophrenia with rates of non-affective and bipolar disorder also higher in migrants. A large cohort study in the UK (AESOP) found a 7-fold increase in mania among African-Caribbeans and black Africans compared to the majority group and a two-fold increase in mania among other migrant groups. Association with age at onset: There is no difference in the age of onset in migrant groups as compared to the majority population. Evidence of Geneenvironment interactions: Family studies of schizophrenia have found a differential morbid risk for schizophrenia in the relatives of UK born African Caribbean probands (15.9\%) compared with the rates in the relatives of white probands $(1.8 \%)$ which suggests a possible geneenvironment interaction.

\section{CANNABIS USE (17)}

There is little doubt that cannabis intoxication is associated with transient psychotic symptoms in some individuals, furthermore case control studies have consistently demonstrated higher rates of cannabis use among patients with schizophrenia as compared with controls. However, controversy remains about whether cannabis is a risk factor for the development of schizophrenia or other psychotic disorders. There are only a few prospective studies examining prior can- 
nabis use and subsequent development of schizophrenia (or other psychotic disorders) but more studies have examined broadly defined psychotic outcomes, such as positive or negative symptoms and their associated disability. Strength of the association: The best evidence for an association between cannabis use in adolescence and subsequent development of schizophrenia, come from a study of Swedish conscripts who were followed up over a 27 years period (18). Cannabis use prior to conscription was associated with higher rates of schizophrenia, estimated odds ratio of 1.5 (95\% confidence interval 1.1-2.0) after adjusting for potential confounders such as disturbed behavior, low IQ, urban upbringing and poor social adjustment. Further, a meta-analysis of prospective cohort studies found similarly high rates of broadly defined psychosis outcome (either psychotic disorder or psychotic symptoms and associated disability) in people who had ever used cannabis compared with no-users, pooled odd ratio of 1.41 (95\% confidence interval 1.20-1.65). There are a number of small studies examining association of cannabis use with symptom dimensions or subtypes of schizophrenia; most have found no association with negative symptoms and cannabis exposure. Dose-response Relationship: In the Swedish conscript study, the effect size increased with frequency of cannabis use and this is a consistent finding in the studies, which used the broader definition of psychosis outcome. Temporal association: It is possible that people with an emerging psychosis or schizophrenic prodrome start using cannabis to reduce their distressing symptoms (reverse causation) and this cannot be fully discounted. However, investigators have generally screened and excluded people who may be experiencing early psychotic symptoms at the start of the prospective studies. Specificity of effect: Studies examining cannabis use and the subsequent development of other psychiatric conditions are small and their findings inconsistent. The evidence to date suggests some specificity of effect for schizophrenia and non-affective psychosis; however, it is too early to call. Consistency of findings \& potential sources of confounding and bias: The association with prior cannabis use and subsequent psychosis is a consistent finding across studies. Adjusting for other drug abuse, known risk factors of schizophrenia and socio demographic variables attenuates the effect but it remains statistically significant. Association with age at onset: One study to date has examined the age of onset and prior cannabis use (retrospectively assessed after onset of psychosis). It found males who used cannabis during adolescence had a significantly lower age of onset. Evidence of Gene-environment interactions: A family study of adolescents with acute psychosis found a ten-fold increase in the morbid risk for schizophrenia in relatives of probands who tested positive for cannabis use, compared to the relatives of probands who tested negative for cannabis. Furthermore, there is evidence that a specific functional polymorphism in the catechol-0-methyltransferase (COMT) gene moderates the effect of adolescent cannabis use. Individuals homozygous for the COMT valine allele of this polymorphism are more likely to exhibit psychotic symptoms and develop schizophreniform disorder in adult life after exposure to cannabis but this adverse effect is not evident in individuals homozygous for the COMT methionine allele. Cannabis use itself is not associated with the COMT valine or methionine allele, suggesting an underlying gene-environmental interaction, (rather than a gene-environment correlation) is operating to increase risk.

\section{STRESSFUL LIFE EVENTS \& EARLY CHILDHOOD TRAUMA}

Stressful life events: many studies report an excess of stressful life events, in the few weeks prior to the onset of psychotic and affective disorders. While these life events may precipitate, the disorder, there is some evidence to suggest they reflect a concatenation of stressful life events over a more sustained period before the onset of psychosis. However, it is difficult to es- 
tablish wither increased stress is a cause or a consequence in the development of psychotic disorder. This is difficult to disentangle with traditional risk factor methodologies; on the other hand, gene-environmental interaction designs may be a more fruitful way of exploring the effect of stress. For example, a study from Greece found stress related to army induction resulted in increased risk of psychotic symptoms but that the specific functional polymorphism in the COMT gene (mentioned above) moderated this effect, as individuals homozygous for the COMT valine allele of this polymorphism were more likely to exhibit psychotic symptoms than individuals homozygous for the COMT methionine allele. Early childhood trauma: describes a range of severe adverse experiences including sexual, physical and emotional abuse and neglect. There are now a modest number of population based studies suggesting that the risk of psychotic experiences is increased in those exposed to early childhood trauma. However, wither childhood trauma is a risk factor for schizophrenia remains controversial and open to question due to a number of conceptual and methodological issues which will require further study.

\section{PRE-MORBID VULNERABILITY INDICATORS/MARKERS}

\section{Pre-morbid indicators: Early Developmental Abnormalities (11)}

As a group, individuals who develop schizophrenia as adults are more likely to manifest subtle motor/language, cognitive abnormalities during childhood and adolescence compared with individuals who do not develop schizophrenia. There does not appear to be any association between developmental abnormalities and prenatal insults, suggesting they may be indicators of genetic liability. Early Developmental Motor Abnormality: A series of large birth cohort studies, have consistently found evidence for delays in attainment of developmental milestones for pre-schizophrenic children. Abnormalities in motor milestones are most conspicuous before the age of two, while receptive language abnormalities are most prominent between the ages of five and fifteen. The relationship between the age of acquiring motor/language milestones and risk of schizophrenia appears to be linear, making a possible 'developmental sub group' unlikely. Furthermore, severe motor/language abnormalities tend to predict an earlier age of onset for schizophrenia. A study from Philadelphia, found increased rates of motor and language abnormalities, not only in the children who later developed schizophrenia, but also in their siblings who remained unaffected, suggesting manifest motor abnormalities may be markers of underlying genetic liability. The early emerging motor and language deficits appear to be relatively specific for schizophrenia (or spectrum disorder). Early Developmental Cognitive Performance: As a group, children who later develop schizophrenia, score lower on standardized measures of intelligence compared to their unaffected counterparts throughout childhood however, the difference widens around the ages of about four to seven. Studies, which compared affected and unaffected siblings, found the pre-schizophrenic children had a lower IQ compared to their unaffected sibling. This deviance in cognitive impairment seems to be relatively specific for schizophrenia (spectrum) disorder and is not present in bipolar disorder. Twin and family studies have shown that the alterations in adult cognitive performance seen in patients and their firstdegree relatives are markers for schizophrenia genetic liability. It is likely that the developmental cognitive alterations similarly reflect the influence of genetic factors associated with schizophrenia. Early developmental social/emotional impairment: As a group, the children who later develop schizophrenia are more likely to show signs of subtle social maladjustment, for example, poor relationships with other children, social isolation, lone play and social anxiety as compared to their peers. However, these subtle social markers are common among children who later develop a broad range of different psychiatric disorders. Predictive Power: While devel- 
opmental abnormalities are common in children who later develop schizophrenia, many preschizophrenic children have normal developmental histories which results in low sensitivity (the ability to predict children who will later develop psychosis correctly). Furthermore, they are relatively prevalent in the general population (estimated at around 15\%) which consequently reduces their specificity (the ability to correctly predict children who will not develop psychosis) which in turn makes them poor predictors of future schizophrenia (positive predictive power estimates range from 3\%-5\%). Developmental abnormalities may be most useful as a possible measurable indicator of genetic liability for schizophrenia

\section{Pre-morbid indicators: psychopathology/vulnerable mental states}

\section{Childhood psychiatric disorders}

The Dunedin Multidisciplinary Health and Development study is a longitudinal follow up of a representative birth cohort of 1037 children born in Dunedin, New Zealand during the year (1972-73). Its strength lies in the fact that it has sequentially assessed not only childhood developmental indices, but also serially collected self-reported psychopathology (ages 11, 13, 15, 18 and 21 years) with further psychiatric interviews having been carried out at age 11 and 26 years. Investigators first examined diagnoses given to individuals across the follow-up period and found over half of those who had been given a major DSM-IV diagnosis at age 26 had also been given a diagnosis at age 11-15 and three quarter had been given a diagnosis before age 18 years. Generally, there was child-to-adulthood syndrome-continuity found for anxiety, depression and antisocial disorders. However, no such syndrome-continuity was evident for adult schizophreniform disorder, which was preceded by a number of different childhood disorders, including anxiety, depression, conduct/oppositional disorder and attention deficit hyperactivity. This group of juvenile diagnoses did not specifically predict adult psychotic disorder but were in fact the harbingers of many different adulthood psychiatric illnesses. Predictive Power: Such findings clearly demonstrate significant psychopathology prior to adult onset psychosis. However, the juvenile categorical diagnoses are demonstrably poor discriminators for what adult psychiatric disorder a child is likely to develop in the future.

\section{Subclinical psychotic experiences}

Subclinical psychotic experiences predict later development of psychotic disorders: Evidence that subclinical psychotic experiences may be vulnerability markers (or even precursors) for the later development of psychotic disorders comes (again) from The Dunedin Multidisciplinary Health and Development study. In further analyses, the investigators examined whether selfreported delusional beliefs and hallucinatory experiences at age eleven would predict schizophreniform psychiatric disorder, fifteen years later (age 26 years) (19). By age 11 years, nearly fifteen percent of the total cohort reported some psychotic experience. Children were grouped according to the strength/frequency of their psychotic experiences (at age 11 years): the majority of children had no psychotic symptoms (control group $(n=654)$ ), an intermediate group had weak symptoms $(n=95)$ and small proportion had symptoms described as strong $(n=12)$. At age 26 years, $2 \%$ of the controls had a diagnosis of schizophreniform disorder, compared with $9.5 \%$ in the weak and $25 \%$ in the strong-symptom-groups. Early psychotic experiences specifically increased the risk for schizophreniform disorder moderately in the weak-symptom-group (odds ratio $5.1,95 \%$ confidence interval $1.7,18.3$ ) and strongly in the strong-symptom-group (odds ratio $16.4,95 \%$ confidence interval 3.9-67.8), compared to controls. While $25 \%$ of the stronggroup had developed schizophreniform disorder, a further 70\% (although not fulfilling the full diagnostic criteria for schizophreniform disorder) still reported some psychotic symptoms (persistence of pre-clinical psychotic experiences) at age 26 years and $90 \%$ had occupational and so- 
cial difficulties. The strong-symptom-group also manifest impairments in motor, language and cognitive ability, suggesting the pervasive and persistent psychotic experiences may be indicators of an underlying ongoing psychotic process, reflecting increased genetic liability. The weaksymptom-group on the other hand did not have such prominent developmental abnormalities, though they had significant receptive language impairment, suggesting a weaker genetic liability. Replication of this transition over time from subclinical psychotic experiences to full-blown psychotic disorder comes from a longitudinal general population study in The Netherlands (The Netherlands Mental Health Survey and Incidence Study; NEMESIS). In order to identify new cases of subclinical psychotic experiences the cohort was followed up for one year. Individuals who reported subclinical psychotic experiences at the 1-year assessment, but not at baseline were considered incident cases. Next, a second-wave follow up (over 2 years) allowed the risk of transition of subclinical to clinical psychotic disorder (both affective and non-affective psychosis) in the incident case group to be compared with the risk in the group of individuals with no clinical or subclinical psychotic experiences (controls). The incident cases were at significantly higher risk of developing psychotic disorders compared to the controls (odds ratio 65.1, 95\% confidence interval 19.4-218.1) and the positive predictive value over 2 years was $8 \%$. There was a dose-response relationship in the association between the number of subclinical psychotic experiences and the transition to clinical psychosis going from a risk of around 30 in individuals who had only one subclinical psychotic experience (odds ratio 27.3, 95\% confidence interval 5.2-143.6) to a much higher risk in individuals who had multiple subclinical experiences (odds ratio 211.2, 95\% confidence interval 51.6-864.1). Characteristics of the subclinical psychotic experiences \& transition to psychosis: In both the Dunedin Cohort and the Nemesis study, transition from subclinical to clinical psychotic disorder was higher in individuals characterized by stronger (persistent) subclinical psychotic experiences. In the Nemesis study, transition was also partly dependent on the emotional context of the subclinical psychotic experience. Most subclinical psychotic experiences are self limiting and transitory: Emerging evidence from general population studies show that subclinical psychotic experiences are relatively common (prevalence of around 15\%). However, most are brief, self-limiting developmental phenomena. The reasons why approximately 15\% of children have subclinical psychotic experiences during development and the others do not, is not fully established, but it may in part reflect the shared genetic liability for psychosis continuously distributed in the general population (20). Towards a dynamic model of psychosis evolution: proneness-persistence-impairment hypothesis. In two large independent general population samples, NEMESIS and EDPD (The early developmental health and incidence survey), investigators examined the hypothesis that these relatively common, subclinical developmental psychotic experiences could become abnormally persistent when synergistically combined with known risk factors for onset of psychotic disorders (the study used cannabis, urban upbringing and developmental trauma). Over a follow-up period of three years, the studies found the rates of subclinical psychotic experiences, which persisted to be relatively low, 26\% (NEMESIS) and 31\% (EDSP), however rates of persistence increased progressively with higher baseline doses of exposure to known risk factors. This suggests that environmental determinants of psychotic disorders may in part operate by driving up the rates of subclinical psychotic experiences, which become persistent in individuals with a genetic liability for psychosis. A dynamic model of psychosis evolution or proneness-persistence-impairment model may best conceptualize this trajectory from developmental expression of psychosis liability (transitory subclinical psychotic symptoms) to more intrusive persistent subclinical psychotic symptoms to significant impairment and dysfunction and the eventual onset of psychotic disorder. Such a model provides a framework for the further investigation of the ontogenesis of psychotic disorders. Predictive power: The psychosis-proneness persistence-impairment-model of psychosis offers a possible insight into the underlying mechanism for development of psycho- 
sis. However, a positive predictive power of around $40 \%$ for persistent subclinical symptoms ability to estimate onset of future psychotic disorders is still too low to be useful as general population screening test.

\section{NEURODEVELOPMENTAL MODEL OF SCHIZOPHRENIA}

The broad neurodevelopmental hypothesis: Posits that schizophrenia is the behavioral outcome of subtle deviances in early brain development, the full effect of which is not manifest until adolescence or early childhood. There is convincing evidence for the broad neurodevelopment model, in agreement with the epidemiological findings for prenatal risk factors and developmental abnormities reviewed in this chapter. Children who later develop schizophrenia also have higher rates of minor physical anomalies indicative of subtle disruption of ectoderm development e.g. craniofacial and dermatoglyphic anomalies and neurological soft signs. Further evidence is the absence of inflammatory reactions in the brains of people with a diagnosis of schizophrenia. While earlier histological studies implicated abnormal neuronal migration during corticogenesis, this was not been borne out with further investigation. However, reduced neuronal size, dendritic arborisation and spine density, in the cortex and hippocampus is a consistent finding across studies. Such findings are important and support a neurodevelopmental model when put in the context of a burgeoning realization that ultra-structural refinement at the synapse is shaped by patterns of environmental input, which occur across the life span, and seems to have functional significance. Such plasticity in the connectivity of neurons is thought to underlie new learning and the development of new schemata and mental skills. Lag between exposure to environmental risk factors and onset of schizophrenia: There is generally a long latency period between exposure (before adulthood) to known risk factors and onset to schizophrenia. Such a delayed effect is consistent with a causal model where the environmental risk factor increases a person's liability, but the full manifestation of the disorder requires the addition or interaction of other complementary (gene or environmental) causal components. Several susceptibility genes for schizophrenia appear to have specific developmental correlates that influence brain development across the life course and it is possibly this variable expression, which forms the basis for the widespread subtle developmental delays and subclinical psychotic experiences seen years before the onset of schizophrenia and later emergence of the full-blown disorder. More over it could potentially help explain the broad variation in age of onset of schizophrenia. Epigenetic Hypothesis Suggests that the onset of schizophrenia is influenced not only by the additive or interactive action of static gene and environmental factors, but via epigenetic dysregulation of gene activity. This model fits well with the familiar (non Mendelian) features of schizophrenia and it is likely that epigenetic strategies will develop the underlying mechanisms of the phenotypic differences in monozygotic twins discordant for schizophrenia. Unlike DNA (Deoxyribonucleic acid) sequencing which usually remains stable over time epigenetics refers to the regulation of gene expressions that are controlled by heritable but potentially reversible changes in DNA methylation and chromatin structure. Epigenetic aberrations (epimutations) may originate from three sources acting individually or in combination 1) inherited through the germline. 2) influence of environmental factors acting across different developmental stages 3) generated via biological stochastic events. Such epigenetic modification of DNA and chromosomal proteins may have a significant impact on regulation of gene expression via age and hormone dependent neorochemical changes rather than the structural changes to the brain. Recent studies suggest that this may be a useful model to btter understand developmental aspects of schizophrenia. A twin case study, of two pairs of monozygotic twins (one pair 
concordant for schizophrenia the other discordant) found a higher degree of DRD2 (dopamine D2 receptor) methylation in the lymphocytes of the affected co-twin as compared to the unaffected twin. Furthermore, the affected twin in the discordant pair was epigenetically more similar to the unrelated affected twins than they were to their own unaffected co-twin. Such epigenetic studies may further our understanding of how abnormally expressed (developmental) genes affect the developmental of the psychosis phenotype.

\section{REFERENCE LIST}

1. Cardo A, Murray R M. The 'classical' genetic epidemiology of schizophrenia. In: Murray R M, Jones P B, Susser E, van Os J, Cannon M, editors. The Epidemiology of schizophrenia. Cambridge: Cambridge University press, 2002.

2. Shih R A, Belmonte P L, Zandi P P. A review of the evidence from family, twin and adoption studies for a genetic contribution to adult psychiatric disorders. International Review of Psychiatry 2004; 14(4):260-283.

3. Riley B, Kendler KS. Molecular genetic studies of schizophrenia. Eurpoean Journal of Human Genetics 2006; 14:669-680.

4. Caspi A, Moffitt T.E. gene-Environment interactions in psychiatry:joining forces with neuroscience. Nature reviews/Neoroscience 2006; 7:583-590.

5. Ottman R. Theoretical Epidemiology: Gene-Environment Interaction:Definitions and study designs. Preventive Medicine 1996; 25:764-770.

6. Rothman KJ, Greenland S. Causation and causal inference. In: Rothman KJ, Greenland S., editors. Modern Epidemiology. Philadelphia: Lippincott-Raven Publishers, 1998: 7.

7. Sipos A, Rasmussen F, Harrison G, Tynelius P, Lewis G, Leon DA et al. Paternal age and schizophrenia: a population based cohort study. BMJ 2004; 329(7474):1070.

8. Davies G, Welham J, Chant D, Torrey EF, McGrath J. A systematic review and meta-analysis of Northern Hemisphere season of birth studies in schizophrenia. [Review] [17 refs]. Schizophrenia Bulletin 2003; 29(3):587-593.

9. Messias E, Kirkpatrick B, Bromet E, Ross D, Buchanan RW, Carpenter WT, Jr. et al. Summer birth and deficit schizophrenia: a pooled analysis from 6 countries. Archives of General Psychiatry 2004; 61(10):985-989.

10. Cannon M, Jones P, Murray R.M. Obstetric Complications and schizophrenia: Historical and Meta-analytic Review. American Journal of Psychiatry 2002; 159:1080-1092.

11. Rapoport JL, Addington AM, Frangou S. The neurodevelopmental model of schizophrenia:update 2005. Molecular Psychiatry 2005; 10:434-449.

12. Brown AS. Prenatal Infection as a risk factor for schizophrenia. Schizophrenia Bulletin 2006; 32(2):200-202.

13. Allardyce J, Boydell J. Review: The wider social Environment and schizophrenia. Schizophrenia Bulletin 2006; 32:592-598.

14. Krabbendam L, van Os J. Schizophrenia and Urbanicity: A Major Environmental Influence-Conditional on Genetic Risk. Schizophrenia Bulletin 2005; 31(4):795-799.

15. Pedersen CB, Mortensen PB. Are the cause(s) responsible for urban-rural differences in schizophrenia risk rooted in families or in individuals? American Journal of Epidemiology 2006; 163(11):971-978.

16. Cantor-Graae E, Selten J. Schizophrenia and Migration: A meta-analysis and Review. The American Journal of Psychiatry 2005; 162(1):12-24.

17. Moore TH, Zammit S, Lingford-Hughes A, Barnes TR, Jones PB, Burke M et al. Cannabis use and risk of psychotic or affective mental health outcomes: a systematic review. Lancet 2007; 370(9584):319-328.

18. Zammit S, Allebeck P, Andreasson S, Lundberg I, Lewis G. Self reported cannabis use as a risk factor for schizophrenia in Swedish conscripts of 1969: historical cohort study.[see comment]. BMJ 2002; 325(7374):1199.

19. Poulton R, Caspi A, Moffitt TE, Cannon M, Murray R, Harrington H. Children's self-reported psychotic symptoms and adult schizophreniform disorder: a 15-year longitudinal study. Archives of General Psychiatry 2000; 57(11):1053-1058. 
20. Cougnard A, Marcelis M, Myin-Germeys I, De GR, Vollebergh W, Krabbendam L et al. Does normal developmental expression of psychosis combine with environmental risk to cause persistence of psychosis? A psychosis proneness-persistence model. Psychological Medicine 2007; 37(4):513-527.

21. Bromet EJ, Naz B, Fochtmann LJ, Carlson GA, Tanenberg-Karant M. Long-term diagnostic stability and outcome in recent first-episode cohort studies of schizophrenia. Schizophrenia Bulletin 2005; 31(3):639-649.

22. Hegarty JD, Baldessarini RJ, Tohen M, Waternaux C, Oepen G. One hundred years of schizophrenia: a metaanalysis of the outcome literature.[see comment]. American Journal of Psychiatry 1994; 151(10):1409-1416.

23. Menezes NM, Arenovich T, Zipursky RB. A systematic review of longitudinal outcome studies of first-episode psychosis. [Review] [93 refs]. Psychological Medicine 2006; 36(10):1349-1362. 
Part 3 


\section{CHAPTER 7}

\section{Defining caseness: Is schizophrenia a valid diagnostic construct?}

Allardyce, J., Gaebel, W., Zielasek, J. and van Os, J. 2007.

Deconstructing Psychosis Conference 2006: The Validity of schizophrenia and alternative approaches to the classification of psychosis.

Schizophrenia Bulletin 33:863-867. 


\section{Deconstructing Psychosis Conference February 2006: The Validity of Schizophrenia and Alternative Approaches to the Classification of Psychosis}

\author{
Judith Allardyce, Wolfgang Gaebel ${ }^{2}$, Jurgen Zielasek ${ }^{2}$, \\ and Jim van Os ${ }^{1}$ \\ ${ }^{2}$ Department of Psychiatry and Psychotherapy, Heinrich-Heine- \\ University, Rhineland State Clinics, Düsseldorf, Germany
}

The DSM V planning process is currently underway and it has once again ignited the debate about the validity of the schizophrenia diagnosis. In this paper, we review the psychometric literature examining the evidence for discontinuity between schizophrenia and normality and the distinction between schizophrenia and other psychotic disorders. We conclude by proposing potential alternative approaches to refining the classification of psychosis.

Key words: psychosis/schizophrenia/classification/ diagnosis/DSM V dimensions

\section{Introduction}

Worldwide, the Diagnostic and Statistical Manual of Mental Disorders, Fourth Edition (DSM-IV) definition of schizophrenia is the most influential in clinical practice and research. ${ }^{1}$ Its clear criterion-based definition facilitates diagnostic agreement (reliability) and communication among practitioners, including comparable statistical reporting of incidence and prevalence rates. ${ }^{2}$ It has high clinical utility, providing nontrivial information about course, outcome, and likely treatment response. ${ }^{3,4}$ However, does this make schizophrenia a valid diagnostic construct?

Clinical usefulness is embedded in the established criteria for nosological validation. ${ }^{5-8}$ A diagnosis is considered useful if its antecedent, biological, social, prognostic, or treatment correlates provide substantial information not contained within the syndrome's definition. ${ }^{6,9}$ If we accept this conflation of utility and validity $D S M$ $I V$ schizophrenia is indeed a robust construct, a model for conceptualizing complex clinical experience, guiding clinical management and predicting outcome.

\footnotetext{
${ }^{1}$ To whom correspondence should be addressed; tel: $+31-43$ 3875-443, fax: + 31-43-3875-444, e-mail: j.vanos@sp.unimaas.nl
}

Clinical utility, however, does not provide information about the fundamental nature and structure of schizophrenia; it does not answer the basic taxonic question "are the correlations of observed clinical characteristics, corroborative of underlying latent phenotypic dimensions (continuous distributions), latent categories (composed of one or more class or subdisorder, each with its own phenotypic presentation) or a mix of the two?"10 That is, usefulness does not provide information on the construct validity of schizophrenia. ${ }^{11}$ If our definition of schizophrenia does not represent a "real" construct in nature, then it will not delineate the true pathology and causal mechanisms underlying psychosis; it will obfuscate etiology. The developers of $D S M-I V$ carefully point out that there is no assumption that each category is a discrete entity. However, they provide an operational definition of schizophrenia presenting the disorder as a condition qualitatively different from health (discontinuity between normality and schizophrenia) and qualitatively different from the other diagnoses (discontinuity between schizophrenia and the related diagnostic categories described in the classification system). Below, we review the evidence for this and discuss alternative approaches to the classification of psychosis.

\section{The distribution of Psychosis in the General Population}

Mounting evidence suggests that, in fact, there are no discrete breaks (demarcations) in the distribution of manifest (positive) symptom indicators of psychosis; delusions and hallucinations seem to have a continuous distribution in the general population. ${ }^{12-24}$ Prevalence estimates, in nonclinical samples, range from $4 \%{ }^{12}$ to $17.5 \%{ }^{21}$ (with methodological differences likely to explain much of this variability) and results from a longitudinal study using the British National Psychiatric Morbidity Survey data found that $4.4 \%$ of the general population reported incident symptoms at 18 -month follow-up. ${ }^{24}$ These rates are not a reflection of unidentified cases "hidden" in the community because only a very small proportion of those reporting positive psychotic symptoms fulfilled diagnostic criteria for $D S M$ nonaffective psychosis. ${ }^{15,21}$

How should we interpret this skewed continuum of positive psychotic symptoms? It may be an artifact, caused by measurement error; the use of lay interview or self-report methods may lower symptom recognition

(C) The Author 2007. Published by Oxford University Press on behalf of the Maryland Psychiatric Research Center. All rights reserved.

For permissions, please email: journals.permissions@oxfordjournals.org. 
thresholds, so studies are measuring psychosis-like experiences, not necessarily related to the clinical features of a true latent category or disease entity. However, even if there is measurement variance between the symptoms elicited in the general population and those from clinical samples, this may be informative, given the fact that psychosis-like symptoms can be conceived as indicators of psychosis proneness, "clinical psychosis" emerging (with higher than expected probability) from the pool of those with psychotic-like features. ${ }^{19,25-27}$

The skewed continuum may be indicative of a latent continuous pathology in the general population. This is consistent with the prevailing view that schizophrenia has a multifactorial etiology where many different genes, which are neither necessary or sufficient causes, and of small effect, interact with each other and with environmental risk factors to cause the disorder, different combinations of risk factors resulting in a gradation of exposure and associated range of presentations from normal through to the clinical disorder. Published work supports this postulated continuity in the risk factor profiles for community-reported symptoms and schizophrenia, though much of the evidence comes from cross-sectional studies where the direction of the associations cannot be determined for exposures that vary over the life course. One study has suggested that there may be some differences in risk factor profiles for psychotic symptoms and clinical psychosis, ${ }^{24}$ though this may in part be a consequence of using current urban residence as a proxy for urban birth and upbringing. If this finding is replicated, it would suggest discontinuity of risk factor profiles, though at a different point (threshold) on the indicator continuum than that suggested by the DSM-IV definition of schizophrenia.

These findings throw into doubt the assumption that schizophrenia exists as a discrete disease entity (categorical latent variable). The requisite population-based studies, using appropriate structural statistical analyses, eg, finite mixture modeling (and its derivates) ${ }^{28,29}$ or coherent cut kinetic methods $\mathrm{s}^{30}$ have not been carried out, so it is still possible that a dichotomous latent construct could underlie the skewed distribution of psychosis indicators. ${ }^{10,31}$ The above approach uses delusions and hallucinations as indicators for the latent (continuous or categorical) construct schizophrenia. It remains possible that they are nothing more than epiphenomena or nonspecific surface symptoms, not core to the pathological process or perhaps even end-stage manifestations of schizophrenia. ${ }^{32}$ If this is the case, then positive psychotic symptoms may not provide adequate coverage of the latent construct whether it exists as a category or dimension in nature.

\section{Schizophrenia: A Disorder Distinct from Other Psychosis?}

The symptoms used to characterize schizophrenia do not define a specific syndrome. Rather, the concept allows a number of different combinations so that many permutations of the defining symptoms are possible (ie, it is a polythetic definition). These symptoms are also found commonly in the other categories of psychosis described in $D S M-I V .^{33}$ Recent studies using psychopathological dimensions (correlations of symptoms determined by factor analysis) suggest that the diagnostic entities are similar with regard to the key symptom dimensions of psychosis. ${ }^{44-36}$ There is, however, variation in the dimensional profiles of different diagnostic categories in that individuals with a diagnosis of schizophrenia score higher in the positive, negative, and disorganized factors, while patients with affective diagnoses score higher in the manic and depressive dimensions and lower in the negative and positive dimensions. ${ }^{4,37}$ This seems to suggest a quantitative variation in symptom dimension scores across current diagnostic categories rather than qualitative differences. The factor solutions across studies have been broadly consistent demonstrating a 5 -factor solution for psychosis-manic, depression, disorganized, positive, and negative (though there may be conflation of the disorganized and negative dimensions in first-onset samples), ${ }^{38}$ reproducibility of this structure strengthens the findings. The true latent structure of psychopathology is still to be clarified, eg, latent class analyses (LCAs) demonstrate similar indicator profiles to those determined by exploratory factor analysis (EFA), ${ }^{39,40}$ confusing our understanding at the latent level. However, the overlapping co-occurrence of dimensions may be indicative of underlying shared risk factors, which are quantitatively rather than qualitatively distinct and continuously expressed. The ambiguous schizoaffective category may simply be the result of trying to demarcate, where in reality no latent discontinuity exists. Reasonable doubt exists about the true latent structure of the psychosis spectrum; therefore, the true appearance of psychosis in nature has yet to be determined.

\section{Alternative Approaches to the Classification of Psychosis}

\section{Refinement of the Diagnostic Category (subtyping)}

The clinical heterogeneity of $D S M-I V$ schizophrenia could be reduced by refinement of the current definition, narrowing the concept, to describe more homogenous symptom clusters or subgroups. ${ }^{41,42}$ One putative categorical subtype is the "deficit syndrome," characterized by enduring primary negative symptoms. ${ }^{43}$ Association studies support the clinical usefulness of this subgroup $^{4-50}$ but tell us little about its construct validity. Does it truly exist in nature as a discrete disease entity (as its definition assumes) or are its observed associations with external validators the result of comparing high scoring individuals with those scoring low on a latent (negative) dimension? If negative symptoms are associated with other important variables in the clinical, neurocognitive, social, or biological domain, any comparison 
of individuals high vs those low in negative symptoms will yield significant group differences regardless of whether or not the true latent structure of negative symptoms is purely dimensional. A recently published study, using coherent cut kinetics, suggests that there may be a latent level discontinuity in negative symptoms within (chronic) schizophrenia, with an estimated base rate of $28 \%-36 \%$. 51 The author's were unable to compare this empirically defined construct with that of deficit syndrome because they had not rated deficit symptoms in their sample. Further support for a possible discrete negative subcategory of schizophrenia comes from a study which used a surface data reduction method (principal components analysis [PCA]) to identify dimensions of psychopathology and found the negative factor scores were bimodally distributed in people with a diagnosis of schizophrenia. ${ }^{4}$ If the PCA factor does represent a latent dimensional construct (which is not necessarily the case), then this suggests a quantitative discontinuity in the negative dimension.

An important limitation of this approach, however, is the use of chronic clinical samples because this can lead to artificial truncation of the symptom severity distribution, which can distort the results by violating the conditional independence assumption needed to obtain unbiased estimates. $^{30,52,53}$

\section{Dimensional Representations}

Another approach that has been used extensively to reduce the clinical heterogeneity seen in schizophrenia is by statistically identifying psychopathological dimensions (groups of symptoms which occur together more often than would be expected by chance alone) using factor analyses. Individuals can then be defined by how high or low they score on the different dimensions, which may coexist. This methodology assumes that the underlying latent structure of psychopathology is continuous. A 3-factor solution has consistently been found in schizophrenia, and when affective symptoms are included, a further 2 factors are identified, namely depressive and mania/excitement. ${ }^{54}$ Expanding this method to include more broadly defined functional psychosis have generally extracted similar 4- or 5-factor solutions. ${ }^{38,55-58}$ Differential associations are consistently found across the symptom dimensions with clinically relevant variables. ${ }^{4,37,59-61}$ Analyses comparing dimensional representations with the traditional diagnostic categories show the dimensions to be more useful at predicting clinical course and treatment needs, though the difference in the discriminative power may be rather small. ${ }^{4,59,62}$ Thus, dimensions seem to add to the information contained within the diagnostic systems, providing assessments that are more detailed and likely to be important particularly in clinical research.

Both these alternative methods for classification (subtypes and dimensions) use latent variable modeling to tap into the underlying structure of psychopathology. However, the approach to date has important limitations. Taxonic analyses have rarely been carried out, prior to the LCA or EFA. Therefore, the decision about which statistical method to use has not been empirically driven but rather reflects the researcher's epistemological stance. If a latent class (taxon) is identified, external analyses (association studies) can be carried out on this subsample of individuals to determine secondary thresholds (subgroups). Failure to restrict these analyses to the taxonic group will introduce unnecessary imprecision into the search for secondary thresholds. On the other hand, if no taxon is identified, it is appropriate to use factor analyses or multidimensional scaling to generate symptom scores, which can be used in external (association) analyses to define diagnostic thresholds. It is important to remember that a latent class can be extracted as a strong factor in EFA (30). Kessler has proposed a 3-tiered approach for the use of structural analyses in the development of psychiatric classification systems. ${ }^{52}$

\section{Search for More Proximal Indicators of Psychosis}

The current definition of schizophrenia and the alternative approaches discussed in this article depend heavily on symptoms and signs that are probably somewhat distal to the underlying pathoetiology. Integration of defining characteristics, more proximal to the pathological process underlying schizophrenia, is likely at some point in the future (reviewed in accompanying articles in this issue). Potentially informative, alternative indicators of psychopathology are the development of standardized and validated functional clinical tests for psychological dysfunction (dysfunctional modules). ${ }^{63}$ A modular concept of psychopathology is grounded in experimental psychological theory, and depends on a model where psychological behavior and brain structure constitute a molar system, made up of identifiable microsubsystems of elementary psychological functions, with corresponding neuronal circuits, distributed networks, ${ }^{64}$ or processing streams. A series or hierarchy of dysfunctional modules would then provide a detailed and individual characterization of an individual patient.

\section{Conclusion/Recommendations}

Two main diagnostic issues arise. First, it is essential to know how the psychosis phenotype or phenotypes exist in nature, in order to study its causes and outcomes. Second, a decision needs to be made about how to derive a useful diagnostic construct from the natural phenotype or phenotypes, so that patients can be usefully identified and treated.

In the short term, there is considerable need for descriptive and latent variable approaches to determine how psychosis is distributed in the general population. 
Identification of naturally occurring taxons, and/or continuous dimensional representations of psychopathology, and their associated course and outcome over time may be clinically very useful.

In the longer term, these descriptive approaches will no doubt be complemented by studies of putative etiological or pathophysiological indicators. However, until this time, the aim of any revision of our classification system should be to optimize clinical utility. The emerging evidence seems to demonstrate that models using both categorical and dimensional representations of psychosis are better discriminators of course and outcome than either model independently. Currently, the most useful approach to classification seems to be the complementary use of categorical and dimensional representations of psychosis.

\section{References}

1. Mezzich JE. International surveys on the use of ICD-10 and related diagnostic systems. Psychopathology. 2002;35: $72-75$.

2. Kendell R, Jablensky A. Distinguishing between the validity and the utility of psychiatric diagnoses. Am J Psychiatry. 2003;160:4-12.

3. Bromet EJ, Naz B, Fochtmann LJ, Carlson GA, TanenbergKarant M. Long-term diagnostic stability and outcome in recent first-episode cohort studies of schizophrenia. Schizophr Bull. 2005;31:639-649.

4. Dikeos DGM, Wickham HMMF, McDonald CMMP, et al. Distribution of symptom dimensions across Kraepelinian divisions. Br J Psychiatry. 2006;189:346-353.

5. Andreasen NC. The validation of psychiatric diagnosis: new models and approaches. Am J Psychiatry. 1995;152:161-162.

6. Kendell RE. Clinical validity. Psychol Med. 1989;19:45-55.

7. Kendler KS. The nosologic validity of paranoia (simple delusional disorder). A review. Arch Gen Psychiatry. 1980;37:699706.

8. Robins E, Guze SB. Establishment of diagnostic validity in psychiatric illness: its application to schizophrenia. Am J Psychiatry. 1970;126:983-987.

9. Spitzer RL. Values and assumptions in the development of DSM-III and DSM-III-R: an insider's perspective and a belated response to Sadler, Hugus and Agich's "on the values in recent American psychiatric classification". J Nerv Ment Dis. 2001;189:351-359.

10. Meehl PE. Bootstraps taxometrics. Solving the classification problem in psychopathology. Am Psychol. 1995;50:266-275.

11. Andreasen NC. Understanding schizophrenia: a silent spring? Am J Psychiatry. 1998;155:1657-1659.

12. Eaton WW, Romanoski A, Anthony JC, Nestadt G. Screening for psychosis in the general population with a self-report interview. J Nerv Ment Dis. 1991;179:689-693.

13. Janssen I, Hanssen M, Bak M, et al. Discrimination and delusional ideation. Br J Psychiatry. 2003;182:71-76.

14. Johns LC, Cannon M, Singleton N, et al. Prevalence and correlates of self-reported psychotic symptoms in the British population. Br J Psychiatry. 2004;185:298-305.

15. Kendler KS, Gallagher TJ, Abelson JM, Kessler RC. Lifetime prevalence, demographic risk factors, and diagnostic val- idity of nonaffective psychosis as assessed in a US community sample. The National Comorbidity Survey. Arch Gen Psychiatry. 1996;53:1022-1031.

16. King M, Nazroo J, Weich S, et al. Psychotic symptoms in the general population of England - a comparison of ethnic groups (The EMPIRIC study). Soc Psychiatry Psychiatr Epidemiol. 2005;40:375-381.

17. Olfson M, Lewis-Fernandez R, Weissman MM, et al. Psychotic symptoms in an urban general medicine practice. Am J Psychiatry. 2002;159:1412-1419.

18. Peters ER, Joseph SA, Garety PA. Measurement of delusional ideation in the normal population: introducing the PDI (Peters et al. Delusions Inventory). Schizophr Bull. 1999; 25:553-576.

19. Poulton R, Caspi A, Moffitt TE, Cannon M, Murray R, Harrington H. Children's self-reported psychotic symptoms and adult schizophreniform disorder: a 15-year longitudinal study. Arch Gen Psychiatry. 2000;57:1053-1058.

20. Tien AY. Distributions of hallucinations in the population. Soc Psychiatry Psychiatr Epidemiol. 1991;26:287-292.

21. van Os J, Hanssen M, Bijl RV, Ravelli A. Strauss (1969) revisited: a psychosis continuum in the general population? Schizophr Res. 2000;45:11-20.

22. van Os J, Hanssen M, Bijl RV, Vollebergh W. Prevalence of psychotic disorder and community level of psychotic symptoms: an urban-rural comparison. Arch Gen Psychiatry. 2001; 58:663-668.

23. Verdoux H, Maurice-Tison S, Gay B, van Os J, Salamon R, Bourgeois ML. A survey of delusional ideation in primarycare patients. Psychol Med. 1998;28:127-134.

24. Wiles NJ, Zammit S, Bebbington P, Singleton N, Meltzer H, Lewis G. Self-reported psychotic symptoms in the general population: results from the longitudinal study of the British National Psychiatric Morbidity Survey. Br J Psychiatry. 2006;188:519-526.

25. Bebbington $\mathrm{P}$, Nayani T. The psychosis screening questionnaire. Int J Methods Psychiatr Res. 1995;5:11-19.

26. Chapman LJ, Chapman JP, Kwapil TR, Eckblad M, Zinser MC. Putatively psychosis-prone subjects 10 years later. J Abnorm Psychol. 1994;103:171-183.

27. McGlashan TH, Johannessen JO. Early detection and intervention with schizophrenia: rationale. Schizophr Bull. 1996;22:201-222.

28. Haertel EH. Continuous and discrete latent structure models for item response data. Psychometrika. 1990;55: 477-494.

29. McCulloch CE, Lin H, Slate EH, Turnbell BW. Discovering subpopulation structure with latent class mixed models. Stat Med. 2002;21:417-429.

30. Lenzenweger MF. Consideration of the challenges, complications, and pitfalls of taxometric analysis. J Abnorm Psychol. 2004;113:10-23.

31. Murphy EA. One cause? Many causes? The argument from the bimodal distribution. J chronic dis. 1964;17:301-324.

32. Goldman-Rakic PS. More clues on "latent" schizophrenia point to developmental origins. Am J Psychiatry. 1995;152: 1701-1703.

33. Kendell RE, Brockington IF. The identification of disease entities and the relationship between schizophrenia and affective psychosis. Br J Psychiatry. 1980;137:324-331.

34. Lindenmayer JP, Brown E, Baker RW, et al. An excitement subscale of the positive and negative syndrome scale. Schizophr Res. 2004;68:331-337. 
35. van Os J, Gilvarry C, Bale R, et al. A comparison of the utility of dimensional and categorical representations of psychosis. UK700 Group. Psychol Med. 1999;29:595-606.

36. van Os J, Gilvarry C, Bale R, et al. Diagnostic value of the DSM and ICD categories of psychosis: an evidence-based approach. UK700 Group. Soc Psychiatry Psychiatr Epidemiol. 2000;35:305-311.

37. Ratakonda S, Gorman JM, Yale SA, Amador XF. Characterization of psychotic conditions. Use of the domains of psychopathology model. [see comment]. Arch Gen Psychiatry. 1998;55:75-81.

38. McGorry PD, Bell RC, Dudgeon PL, Jackson HJ. The dimensional structure of first episode psychosis: an exploratory factor analysis. Psychol Med. 1998;28:935-947.

39. Kendler KS, Karkowski LM, Prescott CA, Pedersen NL. Latent class analysis of temperance board registrations in Swedish male-male twin pairs born 1902 to 1949: searching for subtypes of alcoholism. Psychol Med. 1998;28: 803-813.

40. Murray V, McKee I, Miller PM, et al. Dimensions and classes of psychosis in a population cohort: a four-class, fourdimension model of schizophrenia and affective psychoses. Psychol Med. 2005;35:499-510.

41. Andreasen NC, Olsen S. Negative v positive schizophrenia. Definition and validation. Arch Gen Psychiatry. 1982;39: 789-794.

42. Carpenter WT, Heinrichs DW, Wagman AM. Deficit and nondeficit forms of schizophrenia: the concept. Am J Psychiatry. 1988;145:578-583.

43. Buchanan RW, Carpenter WT. Domains of psychopathology. An approach to the reduction of heterogeneity in schizophrenia. J Nerv Ment Dis. 1994;182:193-204.

44. Fenton WS, McGlashan TH. Antecedents, symptom progression and long term outcome of the deficit syndrome in schizophrenia. Am J Psychiatry. 1994;151:351-356.

45. Heckers S, Goff D, Schacter DL, et al. Functional imaging of memory retrieval in deficit vs nondeficit schizophrenia. Arch Gen Psychiatry. 1999;56:1117-1123.

46. Horan WP, Blanchard JJ. Neurocognitive, social and emotional dysfunction in deficit syndrome schizophrenia. Schizophr Res. 2003;65:125-137.

47. Kirkpatrick B, Buchanan RW. Anhedonia and the deficit syndrome of schizophrenia. Psychiatry Res. 1990;31:25-30.

48. Kirkpatrick B, Ross DE, Walsh D, Karkowski L, Kendler KS. Family characteristics of deficit and nondeficit schizophrenia in the Roscommon Family Study. Schizophr Res. 2000;45:57-64.

49. Kirkpatrick B, Tek C, Allardyce J, Morrison G, McCreadie RG. Summer birth and deficit schizophrenia in Dumfries and Galloway, southwestern Scotland. [see comment]. Am J Psychiatry. 2002;159:1382-1387.

50. Ross DE, Thaker GK, Buchanan RW, et al. Association of abnormal smooth pursuit eye movements with the deficit syndrome in schizophrenic patients. Am J Psychiatry. 1996;153: $1158-1165$.

51. Blanchard JJ, Horan WP, Collins LM. Examining the latent structure of negative symptoms: is there a distinct subtype of negative symptom schizophrenia? Schizophr Res. 2005;77: 151-165.

52. Kessler RC. Epidemiological perspectives for the development of future diagnostic systems. Psychopathology. 2002;35: $158-161$.

53. Ruscio J, Ruscio AM. Clarifying boundary issues in psychopathology: the role of taxometrics in a comprehensive program of structural research. J Abnorm Psychol. 2004;113:24-38.

54. Grube BS, Bilder RM, Goldman RS. Meta-analysis of symptom factors in schizophrenia. Schizophr Res. 1998;31:113-120.

55. Serretti A, Rietschel M, Lattuada E, et al. Major psychoses symptomatology: factor analysis of 2241 psychotic subjects. Eur Arch Psychiatry Clin Neurosci. 2001;251:193-198.

56. Serretti A, Olgiati P. Dimensions of major psychoses: a confirmatory factor analysis of six competing models. Psychiatry Res. 2004;127:101-109.

57. McIntosh AM, Forrester A, Lawrie SM, et al. A factor model of the functional psychoses and the relationship of factors to clinical variables and brain morphology. Psychol Med. 2001;31:159-171.

58. Drake RJ, Dunn G, Tarrier N, Haddock G, Haley C, Lewis S. The evolution of symptoms in the early course of nonaffective psychosis. Schizophr Res. 2003;63:171-179.

59. Peralta V, Cuesta MJ, Giraldo C, Cardenas A, Gonzalez F. Classifying psychotic disorders: issues regarding categorial vs. dimensional approaches and time frame to assess symptoms. Eur Arch Psychiatry Clin Neurosci. 2002;252:12-18.

60. van Os J, Fahy TA, Jones P, et al. Psychopathological syndromes in the functional psychoses: associations with course and outcome. Psychol Med. 1996;26:161-176.

61. van Os J, Gilvarry C, Bale R, et al. A comparison of the utility of dimensional and categorical representations of psychosis. UK700 Group. Psychol Med. 1999;29:595-606.

62. Rosenman S, Korten A, Medway J, Evans M. Dimensional vs. categorical diagnosis in psychosis. Acta Psychiatr Scand. 2003;107:378-384

63. Gaebel W. Saß H. Psychopathologische Methoden und psychiatrische Forschung. Jena, Stuttgart: Gustav Fischer Verlag; 1996:15-28.

64. Shallice T. From Neuropsychology to Mental Structure. Cambridge, England: Cambridge University Press; 1988. 



\section{CHAPTER 8}

\section{Dimensional representations of psychosis}

Allardyce,J., Suppes, T., van Os, J. 2007.

Dimensions and the psychosis phenotype.

International Journal of Methods in Psychiatric Research: 16 S34-S40. 
International Journal of Methods in Psychiatric Research

Int. J. Methods Psychiatr. Res. 16(S1): S34-S40 (2007)

Published online in Wiley InterScience

(www.interscience.wiley.com) DOI: $10.1002 / \mathrm{mpr} .214$

\title{
Dimensions and the psychosis phenotype
}

\author{
JUDITH ALLARDYCE, ${ }^{1}$ TRISHA SUPPES, ${ }^{2}$ JIM VAN OS ${ }^{1,3}$ \\ 1 Department of Psychiatry and Neuropsychology, South Limburg Mental Health Research and Teaching \\ Network, EURON, Maastricht University, Maastricht, The Netherlands \\ 2 Department of Psychiatry, UTSouthwestern Medical Center, Dallas, TX, USA \\ 3 Division of Psychological Medicine, Institute of Psychiatry, London, UK
}

\begin{abstract}
In this paper, we discuss the conceptual background for including a dimensional component to the DSM V diagnoses for psychoses. We review the evidence for a continuous distribution of psychosis like symptoms in the general population and summarise the research validating the clinical usefulness of psychopathological dimensions. We conclude that diagnostic models using both categorical and dimensional representations of psychosis have better predictive validity than either model independently. Dimensions do not appear to be diagnosis specific so a flexible scoring of dimensions across all psychotic and major affective disorders may be potentially more informative than a system where categorical diagnoses are kept artificially dimension-specific. Copyright (C) 2007 John Wiley Eु Sons, Ltd.
\end{abstract}

Key words: Psychosis, schizophrenia, bipolar affective disorder, dimensions, psychosis continuum, psychosis phenotype.

The current Diagnostic and Statistical Manual of Mental Disorders, Fourth Edition (DSM-IV) classification of psychosis stems directly from the systematic clinical observations of Kraepelin (1919), Bleuler (1911) and Schneider (1959) who worked in the large asylums of Western Europe during the late nineteenth and early twentieth century. These institutions provided care for people with severe and debilitating conditions. There are at least two potentially important limitations to a classification system derived from such a selective case sample. First, your clinical experience would be of severe cases in need of treatment, you would understandably conceptualize psychosis as a discrete disease entity, as a categorical construct, distinct from normality. This, however, may not reflect the true distribution of psychosis at the population level.

Second, the observed pattern of psychopathological co-occurrence may actually reflect symptoms, which are independent risk factors for hospital admission becoming conditionally dependent in the institutional setting, a phenomena known as Berkson's fallacy (bias). A community study has shown that positive and nega- tive symptoms are both independently associated with need for care (Maric et al., 2004). Such additive effects could inflate the positive/negative co-occurrence in hospital settings, indicating that the current conceptualization of schizophrenia as a unitary entity with high co-occurrence of positive and negative psychopathological domains may in part be the result of Berkson's bias.

Similar findings apply to the bipolar disorder construct. A general population study has demonstrated independent associations of manic and depressive symptoms, with need for care; while symptom cooccurrence was $17 \%$ in individuals known to services it was only $7 \%$ in those not in the secondary health care system. These independent effects may well inflate depression/mania co-occurrence in institutional settings (Regeer et al., in press).

There is no doubt that the work of Kraepelin, Bleuler and Schneider respectively (and the classification systems which evolved from their insights), has greatly facilitated the acquisition of the knowledge we now have about psychosis. However, the walls of the asylum 
confined their observations, perhaps obscuring the true nature of the psychosis phenotype.

\section{Distribution of psychosis in the general population}

The clinical definitions of psychosis may represent only a minor, possibly biased sample of the total psychosis phenotype present in the general population. This is consistent with the prevailing view that psychosis has a multi-factorial aetiology (similar to that seen in other chronic disorders such a diabetes and cardiovascular disease) where many different genes, which are neither necessary or sufficient causes, and of small effect, interact with each other and with environmental risk factors (Jones and Cannon, 1998). It can be shown that such different combinations of risk factors must result in a gradation of exposure and associated range of different expressions from normal through to clinical psychosis (continuum hypothesis). Mounting support for the continuum hypothesis comes from studies examining (1) the distribution of psychotic symptoms and psychotic proneness in the general population; (2) the pattern of genetic and non-genetic risk factor profiles in non-clinical and clinical samples; (3) the transition from sub-clinical to clinical states over time (up to $25 \%$ in the largest prospective study to date (Poulton et al., 2000)).

The distribution of the positive symptoms of psychosis, delusions and hallucinations, seem to have a continuous distribution in the general population (Eaton et al., 1991; Janssen et al., 2003; Johns et al., 2004; Kendler et al., 1996; King et al., 2005; Olfson et al., 2002; Peters et al., 1999; Poulton et al., 2000; Tien, 1991; van Os et al., 2000a; van Os et al., 2001; Verdoux et al., 1998; Wiles et al., 2006; Spauwen et al., 2003). Prevalence estimates, in non-clinical samples, range from 4\% (Eaton et al., 1991) to $17.5 \%$ (van Os et al., 2000a) (with methodological differences likely to explain much of this variability). High rates do not appear to be secondary to measurement error due to self-report interview techniques, as high rates are also reported using non self-report interviews by clinicians (Poulton et al., 2000; Spauwen et al., 2003). These rates also are not a reflection of unidentified cases 'hidden' in the community, as only a very small proportion of those reporting positive psychotic symptoms fulfilled diagnostic criteria for DSM non-affective psychosis (Kendler et al., 1996; van Os et al., 2000a). That the psychosis phenotype is much more prevalent than previously thought is also supported by recent work showing that the lifetime prevalence of psychotic disorder, when multiple sources are taken into account, exceeds 3\%, much higher than the traditional 0.6\% (Perala et al., 2007). Studies of schizotypy (a personality trait characterized by a proneness to psychotic-like experiences) suggest that it is a quantitative rather than a qualitative trait, on a continuum from normality, through eccentricity, different combinations of schizotypal characteristics, to florid psychosis. Factor analyses of schizotypy extract three or possibly four dimensions: aberrant perceptions and believes, introvert/anhedonia and conceptual disorganization (a factor solution some consider to be similar to that found in schizophrenia). This work suggests that psychosis proneness is a multidimensional continuous construct (Gruzelier, 1996; Mata et al., 2003; Vollema and van den Bosch, 1995).

Mood disturbance similarly appears to have a continuous distribution in the general population. Sub-threshold depression and (hypo)mania, defined as the experience of distinct periods of depressive or (hypo)manic symptoms, which do not fulfill the DSM-III-R/IV diagnostic criteria appear to be common (Angst and Gamma, 2002; Cuijpers et al., 2004) with prevalence rates of up to $13 \%$ for depression, and $9 \%$ for hypomania (Angst and Merikangas, 1997; Angst et al., 2003).

Evidence from longitudinal and cross-sectional studies of risk factors in general support a continuity of risk profiles for subclinical and clinical psychosis (Chapman et al., 1994; Kwapil et al., 1997; Peters et al., 1999; van Os et al., 2000a; van Os et al., 2001; Verdoux et al., 1998). However, one study has demonstrated some differences, which will require further evaluation. Partly, these differences may be due to study design; for example, the study measured the effect of current urban residence on psychotic symptoms, in elderly individuals who are likely to have moved many times and are at very low risk of developing incident psychotic symptoms, rather than studying urban birth/upbringing in young individuals who are most at risk for psychotic symptoms (Wiles et al., 2006).

Finally, longitudinal studies suggest that clinical psychosis emerges, from the pool of those with psychotic-like features, with a much higher than expected frequency (Bebbington and Nayani, 1995; Chapman et al., 1994; Hanssen et al., 2005; Poulton et al., 2000). Non-clinical manic symptoms also appear to represent risk indicators for future clinical manic episodes (Regeer et al., 2006). Interestingly, the probability of developing 
incident bipolar disorder is substantially higher (approximately $7 \%$ ) in individuals with both subclinical manic and psychotic symptoms (Kaymaz et al., 2006).

These studies suggest, that both manic and psychosis phenotypes occur as part of a continuum from normality through to full-blown clinical disorders, that is, they are fundamentally dimensional in nature.

Superimposing categorical diagnoses on latent continuous constructs results in loss of information, but this practice may yield useful short-hand approximations to facilitate communication among clinicians. Potential problems arise, however, if the categories are arbitrary or generated from samples with selection bias (e.g. from the severe end of the spectrum or institutional settings), where they potentially 'misrepresent' the underlying patho-aetiology. This is likely to impede our further understanding of the causes and correlates of psychosis. It may in part explain the current lack of replicable findings in the genetic and biological study of schizophrenia and bipolar disorder.

It is important to keep in mind that although a continuum of psychosis and bipolarity may exist, the diagnosis of need for care and the decision to treat always will remain dichotomous. Need for care results from the interaction between continuous phenotype and the person in terms of, for example, coping, social support, and the level of comorbid developmental impairment

\section{Clinical psychosis: discrete category or psychopathological dimensions}

Categories of psychoses defined in DSM-IV reflect historical notions of severe mental illness observed in institutionalized clinical settings. As discussed earlier, such settings may inflate the co-occurrence of symptoms, obscuring their true latent nature and generating spurious categories. The different psychotic diagnoses overlap in their pre-morbid risk factors, clinical presentations, management needs and outcomes (Murray et al., 2004). This lack of discrimination casts doubt as to how clinically useful the categorical classification systems used today are (McGorry et al., 1998; Toomey et al., 1997), and has resulted in a search for alternative representations of psychoses. One approach is to identify psychopathological dimensions (groups of symptoms which occur together more often than would be expected by chance alone) using exploratory factor analyses (EFA). Individuals can then be defined by how high or low they score on the different dimensions, which may co-exist. The initial work in this area examined the factor structure of the diagnostic category of schizophrenia and found evidence for a three-factor solution (Bilder et al., 1985; Liddle, 1992, 1987; Peralta et al., 1992), extracting positive, negative and disorganized factors. The disorganization factor is the most unstable and least replicable of the three dimensions. However, a two-factor solution does not adequately represent the symptom correlations (Peralta et al., 1994) and the three factor solution may in fact represent higher order factors of many more first order dimensions (Peralta and Cuesta, 1999). A five-factor solution with additional manic and depressive dimensions is found when measures of affective symptoms are included (Lindenmayer et al., 2004). When samples are expanded to include the full spectrum of psychoses, broadly similar five-factors solutions are found, (Dikeos et al., 2006; Kitamura et al., 1995; Lindenmayer et al., 2004; McGorry et al., 1998; McIntosh et al., 2001; Murray et al., 2005; Ratakonda et al., 1998; Serretti et al., 2001; Serretti and Olgiati, 2004), though there may be conflation of the disorganized and negative dimensions especially in first onset samples (McGorry et al., 1998). It seems that the dimensions generated from established cases of psychosis provide reasonably replicable, stable solutions in a variety of settings, diagnostic groups and patient samples; however the factor structure may be less stable around the time of presentation (Drake et al., 2003).

\section{The clinical validity of dimensional representations of psychosis}

Nosological constructs such as psychopathological dimensions should be useful, that is, provide non-trivial information about course, outcome and likely treatment response (Kendell, 1989). A number of studies have examined the association of psychopathological dimensions with various clinically significant characteristics. The most consistent finding is a strong association of the negative dimension with indicators of poor (chronic deteriorating) course (Dikeos et al., 2006; Hollis, 2000; Marengo et al., 2000; van Os et al., 1996; Wickham et al., 2001). The disorganization factor also predicts poor outcome but this is a weaker and less consistent finding. The associations of other dimensions are inconsistent and markedly attenuated after adjustment for diagnosis (Dikeos et al., 2006).

A more informative method of assessing the usefulness of the dimensional approach is to compare the rela- 
tive contribution of the dimensional factor scores and diagnostic categories in predicting the variability of clinically significant characteristics. Such studies consistently show the dimensional representations to be more useful at predicting clinical course and treatment needs, though the difference in the discriminative power may be rather small (Dikeos et al., 2006; Peralta et al., 2002; Rosenman et al., 2003; van Os et al., 1996).

One study has shown that while factor scores add to the predictive power of the diagnostic categories, the diagnostic categories did not increase the predictive power of the dimensional scores. This seems to suggest that the current diagnoses may partition symptom dimensions appropriately. However, models using both categorical and dimensional representations have better discriminative validity, suggesting that the most powerful approach to classification is the complementary use of both categorical diagnoses and dimensional scores (Dikeos et al., 2006).

\section{Practical example}

The following clinical example illustrates how concomitant categorical and dimensional assessment may facilitate patient-care. A 45-year-old man with a 20-year history of bipolar I disorder, presents with mild hypomanic symptoms; reduced need for sleep (2-3 hours less than usual per night), bright and cheerful affect, increased energy, and subjectively feels very productive at work. He has worked as an insurance agent manager of a large company for 10 years, where he is regarded as a conscientious, focused, calm, and task-oriented employee. At interview, he describes plans to reorganize the branch and carry this model throughout the company. This expansive mildly grandiosity does not meet criteria for delusional thinking, but may be on a continuum with delusional grandiose thought. Use of a dimensional measurement would alert the clinician to offer appropriate intervention to avert manic relapse which, if unmanaged, would escalate over a few days. His thought content would move to not only changing his branch, but also going to the head office to tell them how to run the company and telling them he should be CEO. This is one example for the mood case of mania. Similar examples could readily be drawn for patients diagnosed with schizophrenia, mania, or depression.

\section{Potential limitations of a dimensional approach}

To date, with different studies suggesting different numbers of factors or variations in factor composition, there is no definitive model for the symptom dimensions of psychosis, though the five-factor model comprising positive, negative, disorganized, manic and depression dimensions is increasingly being considered to have internal validity. Most exploratory factor analytical studies have used chronic or mixed stage samples, however; if there are psychopathological changes during the course of a disorder (Fenton and McGlashan, 1991; Eaton et al., 1995; Peralta and Cuesta, 2001), samples with different distributions of 'stage of disorder' will yield different symptom dimensions depending on the dominant stage studied. For example, there is no study in the current literature examining the predictive validity of symptom dimensions in first-episode cases of psychosis.

The current diagnostic categories facilitate diagnostic agreement (reliability) and communication among practitioners (Kendell and Jablensky, 2003) and it may be that the introduction of dimensional measures could threaten this. However, what is being proposed initially is a dimensional measure that will complement the categorical diagnosis, perhaps generated from rating scales known to have relatively high inter-rater agreement - for example, the Positive and Negative Syndrome Scale (PANSS) (Kay et al., 1987). Symptom-rating scales are now used routinely in clinical settings to monitor treatment response and relapse and to assess remission. The introduction of a formal dimensional measure in the classification system would hopefully, coordinate and optimize this use.

It is important to decide over which diagnostic categories we measure any proposed psychotic dimensions. The revised classification system could be organized around the presence/absence of psychosis. However, it is quite possible that psychotic symptoms are not fundamental core features of the underlying diseases, but rather, non-specific, perhaps even end-stage manifestations of a number of different pathological processes (Goldman-Rakic, 1995). Crow (1990) has suggested that there exists an aetio-pathological continuum across schizophrenia, schizoaffective disorder, and affective illness, and a recent study of patients with psychotic and non-psychotic bipolar disorder and schizophrenia found a specific association between neurogulin 1 core-at-risk haplotype and 'manic' forms of schizophrenia and 'bipolar' forms of schizophrenia supporting a biological continuum (Green et al., 2005). The literature suggests that substantial 'hidden bipolarity' would be found in patients with unipolar depression 
if a mania dimension were additionally scored in the course of the diagnostic process (Benazzi and Akiskal, 2003; Cassano et al., 2004), and negative symptoms have been demonstrated in patients with bipolar disorder (van Os et al., 2000b). The flexible scoring of dimensions across all psychotic and major affective disorders potentially could be more informative than a system where categorical diagnoses are kept artificially dimension-specific.

\section{Conclusion/recommendations}

It is essential that we understand how the psychosis phenotype or phenotypes exist in nature, in order to study their determinants and outcomes. Further elucidation is likely to come from studies using descriptive and latent variable methodologies to identify fundamental categorical subtypes and/or continuous dimensions of psychopathology. In the future, it is likely these descriptive approaches will be complemented by the inclusion of putative aetiological or pathophysiological indicators.

An ever-increasing number of published studies continue to examine dimensional approaches in schizophrenia and bipolar disorder. Dimensions are not diagnosis-specific, yet current categorical diagnoses require dimensional specificity. In the psychosis literature, affective and non-affective dimensions have been identified in people with psychosis, whereas in individuals with major mood syndromes, psychotic versus non-psychotic domains have been studied. A more productive approach may be to study dimensionality across all mood and psychotic syndromes.

The current evidence supports the complementary use of both categorical and dimensional representations of psychosis. Diagnostic models using both categorical diagnoses and dimensions have better predictive validity than either model independently, and flexible scoring of dimensions across all patients with psychotic and major affective disorders is likely to be especially informative.

\section{References}

Angst J, Gamma A. A new bipolar spectrum concept: a brief review. Bipolar Disorders 2002; Suppl 1: 11-4.

Angst J, Merikangas K. The depressive spectrum: diagnostic classification and course. J Affective Disorders 1997; 45(12): 31-9, discussion 39-40.

Angst J, Gamma A, Benazzi F, Ajdacic V, Eich D, Rossler W. Toward a re-definition of subthreshold bipolarity: epide- miology and proposed criteria for bipolar-II, minor bipolar disorders and hypomania. J Affective Disorders 2003; 732: 133-46.

Bebbington P, Nayani T. The psychosis screening questionnaire. Inter J Methods Psychiatric Res 1995; 5: 11-19.

Benazzi F, Akiskal HS. Refining the evaluation of bipolar II: beyond the strict SCID-CV guidelines for hypomania. J Affective Disorders 2003; 73(1-2): 33-8.

Bilder RM, Mukherjee S, Rieder RO, Pandurangi AK. Symptomatic and neuropsychological components of defect states. Schizophrenia Bull 1985; 11(3): 409-19.

Bleuler E. Dementia Praecox or the group of schizophrenias, 1911 (translated by Zinkin J). New York: International University Press, 1950.

Cassano GB, Rucci P, Frank E, Fagiolini A, Dell'Osso L, Shear MK, Kupfer DJ. The mood spectrum in unipolar and bipolar disorder: arguments for a unitary approach. Am J Psychiatry 2004; 161(7): 1264-9.

Chapman LJ, Chapman JP, Kwapil TR, Eckblad M, Zinser MC. Putatively psychosis-prone subjects 10 years later. J Abnorm Psychol 1994; 103(2): 171-83.

Crow TJ. The continuum of psychosis and its genetic origins. The sixty-fifth Maudsley lecture. Br J Psychiatry 1990; 156: 788-97.

Cuijpers P, de Graaf R, van Dorsselaer S. Minor depression: risk profiles, functional disability, health care use and risk of developing major depression. J Affective Disorders 2004; 79(1-3): 71-9.

Dikeos DGM, Wicham HMMF, McDonald CMMP, Walshe MB, Sigmundsson TM, Bramon EM, Grech AM, Toulopoulou TBMP, Murray RM, Sham PCM. Distribution of symptom dimensions across Kraepelinian divisions. Br J Psychiatry 2006; 189: 346-53.

Drake RJ, Dunn G, Tarrier N, Haddock G, Haley C, Lewis S. The evolution of symptoms in the early course of non-affective psychosis. Schizophrenia Res 2003; 63: 171-9.

Eaton WW, Romanoski A, Anthony JC, Nestadt G. Screening for psychosis in the general population with a self-report interview. J Nerv Ment Dis 1991; 179(11): 689-93.

Eaton WW, Thara R, Federman B, Melton B, Liang KY. Structure and course of positive and negative symptoms in schizophrenia. Arch Gen Psychiatry 1995; 52: 127-34.

Fenton WS, McGlashan TH. Natural history of schizophrenia subtypes. II. Positive and negative symptoms and longterm course. Arch Gen Psychiatry 1991; 48(11): 978-86.

Goldman-Rakic PS. More clues on 'latent' schizophrenia point to developmental origins. Am J Psychiatry 1995; 152: 1701-3.

Green EK, Raybould R, Macgregor S, Gordon-Smith K, Heron J, Hyde S, Grozeva D, Hamshere M, Williams N, Owen MJ, O’Donovan MC, Jones L, Jones I, Kirov G, Craddock N. Operation of the schizophrenia susceptibil- 
ity gene, neuregulin 1, across traditional diagnostic boundaries to increase risk for bipolar disorder. Arch Gen Psychiatry 2005; 62(6): 642-8.

Gruzelier JH. The factorial structure of schizotypy: Part I. Affinities with syndromes of schizophrenia. Schizophrenia Bull 1996; 22(4): 611-20.

Hanssen M, Bak M, Bijl R, Vollebergh W, van Os J. The incidence and outcome of subclinical psychotic experiences in the general population. Br J Clin Psychol 2005; 44(Part 2): 181-91.

Hollis C. Adult outcomes of child- and adolescent-onset schizophrenia: diagnostic stability and predictive validity. Am J Psychiatry 2000; 157(10): 1652-9.

Janssen I, Hanssen M, Bak M, Bijl RV, de Graaf R, Vollebergh W, McKenzie K, van Os J. Discrimination and delusional ideation. Br J Psychiatry 2003; 182: 71-6.

Johns LC, Cannon M, Singleton N, Murray RM, Farrell M, Brugha T, Bebbington P, Jenkins R, Meltzer H. Prevalence and correlates of self-reported psychotic symptoms in the British population. Br J Psychiatry 2004; 185: 298-305.

Jones P, Cannon M. The new epidemiology of schizophrenia. Psychiatric Clinics of North America 1998; 21: 1-25.

Kay SR, Fiszbein A, Opler LA. The positive and negative syndrome scale (PANSS) for schizophrenia. Schizophrenia Bull 1987; 13(2): 261-76.

Kaymaz N, van Os J, de Graaf R, Ten Have M, Nolen W, Krabbendam L. The impact of subclinical psychosis on the transition from subclinical mania to bipolar disorder. J Affective Disorders 98(1-2): 55-64.

Kendell R, Jablensky A. Distinguishing between the validity and the utility of psychiatric diagnoses. Am J Psychiatry 2003; 160: 4-12.

Kendell RE. Clinical validity. Psychological Med 1989; 471: $45-55$.

Kendler KS, Gallagher TJ, Abelson JM, Kessler RC. Lifetime prevalence, demographic risk factors, and diagnostic validity of nonaffective psychosis as assessed in a US community sample. The National Comorbidity Survey. Arch of Gen Psychiatry 1996; 53(11): 1022-31.

King M, Nazroo J, Weich S, McKenzie K, Bhui K, Karlsen S, Stansfeld S, Tyrer P, Blanchard M, Lloyd K, McManus S, Sproston K, Erens B. Psychotic symptoms in the general population of England - a comparison of ethnic groups (The EMPIRIC study). Soc Psychiatry Psychiatric Epidemiol 2005; 40(5): 375-81.

Kitamura T, Okazaki Y, Fujinawa A, Yoshino M, Kasahara Y. Symptoms of psychoses. A factor-analytic study. $\mathrm{Br} \mathrm{J}$ Psychiatry 1995; 166: 236-40.

Kraepelin E. Dementia Praecox and Paraphrenia, 1919 (translated by Barkley RM). New York: Robert E Kreiger, 1971.

Kwapil TR, Miller MB, Zinser MC, Chapman J, Chapman LJ. Magical ideation and social anhedonia as predictors of psychosis proneness: a partial replication. J Abnorm Psychol 1997; 106(3): 491-5.
Liddle PF. Are there more than two syndromes in schizophrenia? A critique of the positive negative dichotomy. $\mathrm{Br} \mathrm{J}$ Psychiatry 1987; 161.

Liddle PF. Syndromes of schizophrenia on factor analysis. Br J Psychiatry 1992; 161: 861.

Lindenmayer JP, Brown E, Baker RW, Schuh LM, Shao L, Tohen M, Ahmed S, Stauffer VL. An excitement subscale of the Positive and Negative Syndrome Scale. Schizophrenia Res 2004; 68: 331-7.

Marengo J, Harrow M, Herbener ES, Sands J. A prospective longitudinal 10-year study of schizophrenia's three major factors and depression. Psychiatry Res 2000; 97(1): 61-77.

Maric N, Myin-Germeys I, Delespaul P, de Graaf R, Vollebergh W, van Os J. Is our concept of schizophrenia influenced by Berkson's bias? Soc Psychiatry Psychiatric Epidemiol 2004; 39(8): 600-5.

Mata I, Gilvarry CM, Jones PB, Lewis SW, Murray RM, Sham PC. Schizotypal personality traits in nonpsychotic relatives are associated with positive symptoms in psychotic probands. Schizophrenia Bull 2003; 29(2): 273-83.

McGorry PD, Bell RC, Dudgeon PL, Jackson HJ. The dimensional structure of first episode psychosis: an exploratory factor analysis. Psychological Med 1998; 28: 935-47.

McIntosh AM, Forrester A, Lawrie SM, Byrne M, Harper A, Kestelman JN, Best JJ, Johnstone EC, Owens DG. A factor model of the functional psychoses and the relationship of factors to clinical variables and brain morphology. Psychological Med 2001; 31: 159-71.

Murray RM, Sham P, van Os J, Zanelli J, Cannon M, McDonald C. A developmental model for similarities and dissimilarities between schizophrenia and bipolar disorder. Schizophrenia Res 2004; 71(2-3): 405-16.

Murray V, McKee I, Miller PM, Young D, Muir WJ, Pelosi AJ, Blackwood DH. Dimensions and classes of psychosis in a population cohort: a four-class, four-dimension model of schizophrenia and affective psychoses. Psychological Med 2005; 35(4): 499-510.

Olfson M, Lewis-Fernandez R, Weissman MM, Feder A, Gameroff MJ, Pilowsky D, Fuentes M. Psychotic symptoms in an urban general medicine practice. Am J Psychiatry 2002; 159(8): 1412-9.

Perala J, Suvisaari J, Saarni SI, Kuoppasalmi K, Isometsa E, Pirkola S, Partonen T, Tuulio-Henriksson A, Hintikka J, Kieseppa T, Harkanen T, Koskinen S, Lonnqvist J. Lifetime prevalence of psychotic and bipolar I disorders in a general population. Arch Gen Psychiatry 2007; 64: 19-28.

Peralta V, Cuesta MJ. Dimensional structure of psychotic symptoms: an item-level analysis of SAPS and SANS symptoms in psychotic disorders. Schizophrenia Res 1999; 38(1): 13-26.

Peralta V, Cuesta MJ. How many and which are the psychopathological dimensions in schizophrenia? Issues influ- 
encing their ascertainment. Schizophrenia Res 2001; 49(3): 269-85.

Peralta V, Cuesta MJ, de Leon J. An empirical analysis of latent structures underlying schizophrenic symptoms: a four-syndrome model. Biol Psychiatry 1994; 36: 726-36.

Peralta V, de Leon J, Cuesta MJ. Are there more than two syndromes in schizophrenia? A critique of the positivenegative dichotomy. [see comment]. Br J Psychiatry 1992; 161: 335-43.

Peralta V, Cuesta MJ, Giraldo C, Cardenas A, Gonzalez F. Classifying psychotic disorders: issues regarding categorial vs. dimensional approaches and time frame to assess symptoms. Eur Arch Psychiatry Clin Neurosci 2002; 252(1): 12-8.

Peters ER, Joseph SA, Garety PA. Measurement of delusional ideation in the normal population: introducing the PDI (Peters et al. Delusions Inventory). Schizophrenia Bull 1999; 25(3): 553-76.

Poulton R, Caspi A, Moffitt TE, Cannon M, Murray R, Harrington $H$. Children's self-reported psychotic symptoms and adult schizophreniform disorder: a 15-year longitudinal study. Arch Gen Psychiatry 2000; 57(11): 1053-8.

Ratakonda S, Gorman JM, Yale SA, Amador XF. Characterization of psychotic conditions. Use of the domains of psychopathology model. Arch Gen Psychiatry 1998; 55: $75-81$.

Regeer E, Krabbendam L, Ten Have M, Olen WA, van Os J. Berkson's Bias and the mood dimensions of bipolar disorder. Soc Psychiatry Psychiatric Epidemiol (in press).

Regeer EJ, Krabbendam L, de Graaf R, Have MT, Nolen NWA, van Os J. A prospective study of the transition rates of subthreshold (hypo)mania and depression in the general population. Psychological Med 2006; 36: 619-627.

Rosenman S, Korten A, Medway J, Evans M. Dimensional vs. categorical diagnosis in psychosis. Acta Psychiatrica Scandinavica 2003; 107: 378-84.

Schneider K. Clinical Psychopathology. New York: Grune \& Stratton, 1959.

Serretti A, Olgiati P. Dimensions of major psychoses: a confirmatory factor analysis of six competing models. Psychiatry Res 2004; 127: 101-9.

Serretti A, Rietschel M, Lattuada E, Krauss H, Schulze TG, Muller DJ, Maier W, Smeraldi E. Major psychoses symptomatology: factor analysis of 2241 psychotic subjects. Eur Arch Psychiatry Clin Neurosci 2001; 251: 193-198.
Spauwen J, Krabbendam L, Lieb R, Wittchen HU, van Os J. Sex differences in psychosis: normal or pathological? Schizophrenia Res 2003; 62: 45-49.

Tien AY. Distributions of hallucinations in the population. Soc Psychiatry Psychiatric Epidemiol 1991; 26(6): 287-92.

Toomey R, Kremen WS, Simpson JC, Samson JA, Seidman LJ, Lyons MJ, Faraone SV, Tsuang MT. Revisiting the factor structure for positive and negative symptoms: evidence from a large heterogeneous group of psychiatric patients. Am J Psychiatry 1997; 154: 371-7.

van Os J, Hanssen M, Bijl RV, Ravelli A. Strauss (1969) revisited: a psychosis continuum in the general population? Schizophrenia Res 2000a; 45: 11-20.

van Os J, Hanssen M, Bijl RV, Vollebergh W. Prevalence of psychotic disorder and community level of psychotic symptoms: an urban-rural comparison. Arch Gen Psychiatry 2001; 58(7): 663-8.

van Os J, Gilvarry C, Bale R, van Horn E, Tattan T, White I, Murray R. Diagnostic value of the DSM and ICD categories of psychosis: an evidence-based approach. UK700 Group. Soc Psychiatry Psychiatric Epidemiol 2000b; 35(7): 305-11.

van Os J, Fahy TA, Jones P, Harvey I, Sham P, Lewis S, Bebbington P, Toone B, Williams M, Murray R. Psychopathological syndromes in the functional psychoses: associations with course and outcome. Psychological Med 1996; 26: 161-76.

Verdoux H, Maurice-Tison S, Gay B, van Os J, Salamon R, Bourgeois ML. A survey of delusional ideation in primarycare patients. Psychological Med 1998; 28(1): 127-34.

Vollema MG, van den Bosch RJ. The multidimensionality of schizotypy. Schizophrenia Bull 1995; 21(1): 19-31.

Wickham H, Walsh C, Asherson P, Taylor C, Sigmundson T, Gill M, Owen MJ, McGuffin P, Murray R, Sham P. Familiarity of symptom dimensions in schizophrenia. Schizophrenia Res 2001; 47(2-3): 223-32.

Wiles NJ, Zammit S, Bebbington P, Singleton N, Meltzer H, Lewis G. Self-reported psychotic symptoms in the general population: results from the longitudinal study of the British National Psychiatric Morbidity Survey. Br J Psychiatry 2006; 188: 519-26.

Correspondence: Judith Allardyce, Department of Psychiatry and Neuropsychology, South Limburg Mental Health Research and Teaching Network, EURON, Maastricht University, Maastricht, The Netherlands.

Email: j.allardyce@sp.unimaas.nl 



\section{CHAPTER 9}

\section{Diagnostic Utility: categorical and dimensional representations of psychosis comparison of their discriminate validity with known risk factors.}

Allardyce, J., McCreadie, R.G, Morrison, G., van Os, 2007

Do symptom dimensions or categorical diagnoses best discriminate between known risk factors for psychosis.

Social Psychiatry and Psychiatric epidemiology 42:429-437. 


\section{Do symptom dimensions or categorical diagnoses best discriminate between known risk factors for psychosis?}

Accepted: 6 February 2007 / Published online: 14 May 2007

Abstract Objective To describe symptom dimensions of psychosis using detailed psychopathological information from epidemiologically defined incident cases which include the full spectrum of functional psychosis across all age ranges. Then, assess the comparative usefulness of the dimensional and categorical representations of psychosis in discriminating between demographic and pre-morbid risk factors. Method A total of 464 incident cases of psychosis assessed with OPCRIT (Operational Checklist for Psychotic Symptoms) were included in an exploratory factor analysis. Using Regression analyses we modelled the associations of the dimensional and categorical representations of psychosis with antecedent validating variables and compared the subsequent models using the likelihood ratio test. Results Factor analysis produced five-symptom dimensions, manic, disorganisation, depressive, delusional and auditory hallucinatory symptoms, explaining $58 \%$ of the total variance. Different dimensions were differentially associated with the pre-morbid risk factors. Neither the dimensional nor the categorical representations on their own were sufficient to explain associations with the antecedent validating variables. Conclusion Neither the dimensional or the diagnostic representation of psychosis was superior in discriminating between known risk factors, combining dimensional measures with categorical diagnoses will probably be more informative in determining the causes and correlates of psychosis.

J. Allardyce $(\bowtie) \cdot J$. van Os

Dept. of Psychiatry and Neuropsychology

Maastricht University

P.O. Box 616 (location DOT10)

6200 MD Maastricht, The Netherlands

E-Mail: j.allardyce@clinmed.gla.ac.uk

J. Allardyce · R.G. McCreadie - G. Morrison

Dept. of Clinical Research

Crichton Royal Hospital

Dumfries (SCO) DG1 4TG, UK
Key words first episode psychosis - exploratory factor analyses - dimensions - classification systems - premorbid risk factors

\section{Introduction}

The different psychotic diagnoses overlap in their pre-morbid risk factors, clinical presentations, management needs and outcomes. This lack of discrimination casts doubt as to how clinically useful the categorical classification systems used today are [23, 42 ], and has resulted in a search for alternative representations of psychoses. One approach is to identify psychopathological dimensions (groups of symptoms which occur together more often than would be expected by chance alone) using exploratory factor analyses (EFA). Individuals can then be defined by how high or low they score on the different dimensions, which may co-exist.

To date there is no definitive model for the symptom dimensions of psychosis, different studies suggesting different numbers of factors or variations in factor composition. These inconsistent findings may be due to differences in methodology [31], most EFA work having studied chronic or mixed stage samples however, if there are psychopathological changes during the course of a disorder [13, 15, 31], samples with different distributions of 'stage of disorder' will yield different symptom dimensions depending on the dominant stage studied. In addition the majority of work has examined patients within the traditional diagnostic categories, particularly schizophrenia, however the dimensions described do not appear specific to any one category. [22, 33]. Therefore, to develop our understanding of the dimensional representation of psychosis we need to study symptoms at specific stages and across the range of psychotic diagnoses. 
Important in the design of an EFA is the choice of variables to include in the study. Concept driven instruments are likely to produce dimensions which reflect their underlying constructs, to avoid this bias recent studies have used OPCRIT (The Operational Checklist for Psychotic Disorders) [6, 7, 25, 28, 30, 3537] which has no theoretical assumptions underlying its design and covers a comprehensive range of psychopathology, including affective symptoms. Finally, classification systems should be useful, that is provide non-trivial information about biological, social, prognostic and treatment correlates and we should evaluate competing systems using these associations. $[19,34]$

\section{Aims of the study}

(1) To describe symptom dimensions using detailed psychopathological information rated on OPCRIT, from incident cases of psychosis. (2) Assess the nosological usefulness of the symptom dimensions by modelling their associations to demographic and premorbid risk factors. (3) Examine the comparative usefulness of the dimensional and categorical representations of psychosis in discriminating between demographic and pre-morbid risk factors.

\section{Methods}

Sample

\section{Catchment area}

Dumfries and Galloway is a geographically well-defined area in South-West Scotland. It has a stable population of around 147,000, of whom $99.5 \%$ are white. Psychiatric services are provided by one hospital and its associated community services. There is little or no private health care.

\section{Patient identification}

Case ascertainment has been described in detail elsewhere [1]. Briefly, we identified all patients who came in to contact with psychiatric services in Dumfries and Galloway over a 20 -year period (1979-1998) who were given a clinical diagnosis of schizophrenia, schizoaffective disorder, delusional disorder, mania, drug induced psychotic disorder; acute, transient or unspecified psychotic disorder. Patients were excluded if they were not resident in Dumfries \& Galloway, had presented previously with a psychotic episode out with the study period or had a demonstrable medical condition, which would account for their symptoms.

\section{Patient characteristics}

A total of 464 patients had a first episode of psychosis during the study period. There were $210(45.26 \%)$ males and $254(54.74 \%)$ females with a median age at presentation of 36 (inter-quartile range 25-55). Males and females showed a significant difference in the age of onset, the median age for males was 33 and for females it was 39.5 (Mann-Whitney, $p<0.001$ )

\section{Assessment of psychopathology}

\section{Symptom ratings}

The case records which were recognised to be comprehensive and above average at the most recent Royal College of Psychiatrists training approval visit and contain medical, informant, nursing, social work and occupational therapy notes and all correspondence for the 464 patients identified as having a first episode of psychosis were examined and OPCRIT was completed. OPCRIT is a checklist containing 90 items, exploring socio-demographic information, for psychotic and affective symptoms (we used binary (present/absent) ratings for the EFA). It was designed with case note review in mind and it has an associated computer algorithm allowing classification with different diagnostic systems. OPCRIT has established reliability $[21,24,44]$ and is a convenient, widely used and validated assessment tool $[4,9]$. Two experienced psychiatrists working independently (JA, GM) completed the OPCRIT checklist for the year of presentation and were blind to the clinically coded diagnosis.

\section{Selection of symptoms for entry into factor analysis}

We excluded items from OPCRIT not related to phenomenology, or where it would be difficult to determine if they were primary or in fact, secondary to medication (initial/middle insomnia, excessive sleep, reduced concentration, slowed activity, loss of energy, increased appetite, weight gain/loss). Items that could make the factor analytic procedures computationally unstable and therefore unreliable were: excluded when their variance was close to zero (catatonia, incoherent speech, diurnal variation, loss of pleasure, excessive self-reproach, early morning wakening, delusions of guilt, delusions of poverty, increased sociability, negative formal thought disorder, lack of insight) or when reliability was poor (primary delusions): used to create composite variables where there was high collinearity (a). Thought alienation: thought broadcasting, thought insertion and thought withdrawal) (b) blunted affect and flattened affect (c) Third person auditory hallucinations, thought echo and running commentary voices). There were 28 items entered into the EFA (see Table 1).

\section{Reliability of opcrit ratings}

Good inter-rater reliability for OPCRIT generated ICD10 and DSM IV diagnosis of schizophrenia has been demonstrated for this sample [1]. The raters (JA, GM) independently scored 45 randomly selected cases to calculate a kappa statistic of agreement for individual items. 
Table 15 -factor solution (with promax rotation)

\begin{tabular}{|c|c|c|c|c|c|c|c|}
\hline & $\begin{array}{l}\text { Kappa co-efficient } \\
\text { Inter-rater reliability }\end{array}$ & Manic & Disorganised & Depressive & Delusional & $\begin{array}{l}\text { Auditory } \\
\text { hallucinations }\end{array}$ & Communality \\
\hline Bizarre behaviour & 0.87 & 0.33 & 0.49 & 0.07 & -0.20 & 0.20 & 0.43 \\
\hline Excessive activity & 0.84 & 0.86 & -0.05 & 0.03 & 0.22 & 0.02 & 0.79 \\
\hline Reckless activity & 0.62 & 0.80 & -0.04 & -0.08 & 0.16 & -0.05 & 0.66 \\
\hline Distractibility & 0.54 & 0.80 & 0.21 & -0.14 & 0.10 & 0.06 & 0.71 \\
\hline Reduced need for sleep & 0.76 & 0.77 & -0.27 & 0.20 & 0.19 & 0.20 & 0.78 \\
\hline Agitated behaviour & 0.61 & 0.76 & 0.01 & -0.44 & -0.15 & 0.12 & 0.80 \\
\hline Speech difficult to understand & 0.80 & 0.13 & 0.86 & 0.04 & 0.15 & -0.07 & 0.78 \\
\hline Positive formal thought disorder & 0.88 & -0.25 & 0.85 & 0.31 & 0.15 & -0.11 & 0.92 \\
\hline Pressured speech & 0.87 & 0.70 & 0.02 & 0.24 & 0.19 & -0.16 & 0.61 \\
\hline Thoughts racing & 0.78 & 0.77 & -0.07 & 0.14 & 0.10 & -0.02 & 0.63 \\
\hline Inappropriate affect & 0.91 & 0.02 & 0.63 & 0.02 & 0.16 & -0.01 & 0.42 \\
\hline Elated mood & 0.83 & 0.81 & 0.01 & 0.03 & 0.14 & -0.16 & 0.70 \\
\hline Irritable mood & 0.81 & 0.81 & -0.10 & -0.28 & 0.01 & -0.10 & 0.75 \\
\hline Dysphoria & 0.78 & 0.04 & -0.13 & -0.90 & 0.11 & -0.18 & 0.87 \\
\hline Suicidal ideation & 0.73 & -0.04 & -0.02 & -0.70 & -0.03 & -0.03 & 0.55 \\
\hline Persecutory delusions & 0.87 & -0.23 & -0.09 & -0.02 & -0.71 & -0.03 & 0.56 \\
\hline Well organised delusions & 0.69 & -0.25 & -0.27 & 0.12 & -0.81 & -0.14 & 0.82 \\
\hline Increased self-esteem & 0.76 & 0.73 & 0.17 & 0.09 & -0.01 & -0.22 & 0.61 \\
\hline Grandiose delusions & 0.69 & 0.67 & 0.29 & 0.10 & 0.19 & -0.03 & 0.57 \\
\hline Delusions of influence & 0.69 & -0.33 & 0.19 & 0.07 & -0.28 & -0.01 & 0.23 \\
\hline Bizarre delusions & 0.70 & -0.02 & 0.53 & -0.03 & 0.15 & 0.07 & 0.31 \\
\hline Widespread delusions & 0.86 & -0.17 & -0.02 & 0.07 & -0.73 & -0.02 & 0.57 \\
\hline Delusions of passivity & 0.88 & -0.16 & 0.39 & -0.10 & 0.08 & 0.17 & 0.22 \\
\hline Abusive/accusatory voices & 0.70 & -0.23 & -0.17 & -0.07 & 0.24 & 0.68 & 0.61 \\
\hline Other hallucinations & 0.67 & -0.25 & 0.17 & -0.33 & 0.15 & -0.24 & 0.28 \\
\hline Restricted or blunted affect & 0.70 & -0.54 & 0.33 & -0.16 & 0.17 & -0.11 & 0.46 \\
\hline Thought alienation & 0.81 & -0.15 & 0.34 & -0.16 & -0.19 & 0.18 & 0.20 \\
\hline Third person auditory hallucinations & 0.92 & -0.08 & -0.01 & 0.24 & 0.05 & 0.96 & 0.98 \\
\hline
\end{tabular}

\section{Exploratory factor analysis}

\section{Statistical procedure for fitting the common factor model on the tetrachoric correlation matrix}

It is appropriate to use EFA to identify the latent symptom dimensions underlying psychotic presentations. EFA is based on the common factor model, which assumes that a variable in a battery of measured variables is a linear function of one or more common factors and one unique factor. Common factors are latent constructs that influence more than one variable and account for the observed correlations seen among them. The Unique factor (with a specific and an error component) influences only one variable and does not account for observed correlations. The common factor model estimates the pattern of association between the common factors and each variable and indexes them as factor loadings. As the OPCRIT symptom scale was of a binary nature, factor analytical methods appropriate to this type of were applied using TESTFACT 4 computer programme [45]. We did a principal factor analysis with communality iterations on the tetrachoric correlation matrix of the 28 OPCRIT derived psychopathological ratings.

\section{Determination of the number of factors to extract}

Determination of the number of factors to include in the model was guided by the scree test. The basic rationale for the scree test is that the battery of variables is measuring a limited number of factors well and a larger number of trivial, specific and error factors less well. Thus, the prominent factors account for most of the variance whereas the other factors are quite numerous but small [16]. By computing the eigenvalues for the correlation matrix and plotting them in descending value along the ordinate with the eigenvalue number as the abscissa, a straight edge can then be laid across the bottom portion of the eigenvalues where they form an approximately straight line. The point where the eigenvalue plot curves above the straight line formed by the smaller values gives the number of factors [8]. Review of the clinical meaningfulness and interpretability of the factors extracted and the reproducibility of these factors complemented the scree test.

\section{Factor rotation to aid interpretation}

To aid interpretation of the solution, factors are rotated in multidimensional space to find a solution with the best simple structure (i.e., where each factor is defined by a subset of variables that have large loadings relative to the other variables and in where each variable loads highly on only a subset of common factors). Two forms of factor rotation were used (1) Promax, which allows factors to be oblique (correlated), which we consider the appropriate method (2) Varimax, which produces orthogonal (uncorre- 
lated) factor solution has been extensively used in previous published studies. We wished to determine wither the choice of rotational method significantly effects the factor solution.

\section{Examination of the stability of the factor solution}

A prime criterion for any rotated factor solution is that it should produce the same factors when random sets of individuals are drawn from the underlying population. If it cannot, the solution is of no value [16]. To assess the stability of the obtained factor solution we randomly split the sample in two and factor analysed the halves separately. We then assessed the congruence of the solutions using Pearson product moment and one-way random effects intraclass correlation co-efficients for the loadings in the corresponding factors.

\section{Factor score estimation and the distribution of dimensions within diagnostic classes}

For each patient we generated (1) OPCRIT derived diagnoses for The International Classification of Diseases, 10th revision (ICD-10) [46], Research Diagnostic Criteria (RDC) [38] and The Diagnostic and Statistical Manual of Mental Disorders, 4th edition (DSM-IV) [3] and (2) factor score estimates for each factor. An individual was considered to have scored 'high' on a particular dimension if their score was above the upper tertile. 'High' score profiles were created for each diagnostic category.

\section{The relationship of dimensions and categories to known premorbid risk factors}

Using STATA statistical programme, release 9 [39], we fitted a series of linear/logistic regression models, with each pre-morbid validator as the dependent variable (1. gender, 2. age at presentation, 3. marital status, 4 . unemployment at presentation, 5. poor premorbid work adjustment, 6 . poor pre-morbid social adjustment 7. Drug and alcohol misuse within one year of onset of symptoms, 8. identified psychosocial stressor, 9. family history of schizophrenia in first or second-degree relatives and 10. family history of other psychiatric disorders severe enough to warrant referral to specialist services) and the dimensional scores as the independent variables.

We repeated these analyses, entering the diagnostic categories (DSM IV) as independent variables and again, with both dimensional and categorical schemata (the full model) as independent variables. Using the Likelihood ratio test we compared the series of analyses containing the full model with models constrained by dropping the categorical and the dimensional components in turn. [18].
Table 2 Factor correlations for analysis shown in

\begin{tabular}{lrrrrr}
\hline & Manic & Disorganised & Depressive & Delusional hallucinations \\
\hline Manic & 1.000 & & & & \\
Disorganised & -0.129 & 1.000 & & & \\
Depression & 0.207 & -0.323 & 1.000 & & \\
Delusional & 0.339 & -0.259 & 0.275 & 1.000 & \\
hallucinations & -0.296 & 0.320 & -0.362 & -0.292 & 1.000 \\
\hline
\end{tabular}

\section{Results}

\section{Reliability of the opcrit ratings}

All symptom ratings showed a good to excellent agreement except distractibility, which showed moderate agreements (Table 1). Similarly, satisfactory agreement was shown for the demographic and risk factors which all had a kappa above 0.7 except for psychosocial stressor (0.64) [2].

\section{Factor structure}

There were eight factors with eigenvalues greater than unity; the scree test suggested a 5 -factors solution, explaining $58 \%$ of the variance but 4,5 and 6 factor solutions were examined. The 5 -factor solution made most substantive sense and is presented in detail here. Varimax and Promax rotated solutions gave similar results (Promax solution presented) (Table 1). The factors were mildly correlated (Table 2) and were predominately univocal (items only load highly on to one common factor). Factor 1 is aligned to the manic symptoms of psychosis with principal loadings for excessive activity (0.86), reckless activity $(0.80)$, distractibility $(0.80)$, reduced need for sleep $(0.77)$, agitated behaviour (0.76) pressured speech (0.70), thoughts racing (0.77), elated mood (0.81), irritable mood (0.81), increased self-esteem (0.73) and mood congruent grandiose delusions (0.67). Restricted/ blunted affect $(-0.54)$ had an opposite (negative) substantial loading i.e., a reverse relationship with the positively loaded "manic" symptoms. Factor 2 included features of 'disorganisation' with the principal loadings; speech difficult to understand (0.86), positive formal thought disorder (0.85) inappropriate affect $(0.63)$ in conjunction with bizarre behaviour (0.49) and bizarre delusions (0.53). Factor 3 we have described as a 'depression factor' with agitated behaviour $(-0.44)$ dysphoria $(-0.71)$ and suicidal ideation $(-0.81)$ loading on to it. All substantial loading on this dimension (and factor 4) are negative; this does not refer to the polarity of the constructs by themselves, but rather the sign is relational to the other loadings on that factor. It would be quite valid to reverse all signs on this factor (and the signs of the correlations between factors) to aid interpretation [16]. Factor 4, included the 'non-bizarre/non-mood 
Table 3 Distribution of opcrit generated diagnoses

\begin{tabular}{|c|c|c|c|c|c|c|c|c|c|}
\hline \multirow[b]{2}{*}{ Diagnosis } & \multicolumn{3}{|l|}{ DSM-IV } & \multicolumn{3}{|l|}{ ICD-10 } & \multicolumn{3}{|l|}{$\mathrm{RDC}$} \\
\hline & $N(\%)$ & Median age & Percent male & $N(\%)$ & Median age & Percent male & $N(\%)$ & Median age & Percent male \\
\hline Schizophreniform & 89 (19.2) & 33 & 43.8 & - & & & - & & \\
\hline Schizophrenia & $113(24.4)$ & 33 & 54.0 & $304(65.5)$ & 34 & 51 & $278(59.9)$ & 36 & 49.3 \\
\hline Mania & 67 (14.4) & 39 & 38.8 & $51(10.1)$ & 37 & 39.2 & $58(12.5)$ & 40.5 & 37.9 \\
\hline Major Depression & $9(2)$ & 42 & 11.1 & $4(0.9)$ & 28.5 & 25 & $9(1.9)$ & 37 & 33.3 \\
\hline Delusional disorder & $32(6.9)$ & 72.5 & 31.3 & - & & & - & & \\
\hline Unspecified & $140(30.4)$ & 35.5 & 49.3 & $104(22.4)$ & 28.5 & 32.7 & $63(13.8)$ & 37 & 45.3 \\
\hline Schizoaffective & $3(0.7)$ & 31 & 33.3 & $1(0.2)$ & - & - & 53 (11.4) & 33 & 35.9 \\
\hline Not classified & $11(2.4)$ & 65 & 27.3 & - & & & $2(0.43)$ & 23 & 0 \\
\hline
\end{tabular}

congruent delusional symptoms'; persecutory delusions $(-0.71)$, well organised delusions $(-0.81)$, widespread delusions $(-0.73)$. Factor 5 was a second positive factor consisting of 'auditory hallucinations' abusive/accusatory/persecutory voices (0.68) and the composite variable of third person auditory hallucinations; thought echo and running commentary (0.96).

\section{Stability of factors across split halves of the sample}

The Pearson's product moment and the one-way random effects intra-class correlation for factor loadings across the two halves produced almost identical results therefore we have only reported the Pearson's correlation. The 5-factor solution produced the most similar results across the two halves, with factor loading correlations of $0.95,0.73,0.74,0.89$ and $0.62(p<0.001)$.

\section{Opcrit generated diagnoses}

The commonest diagnoses were schizophrenia and unspecified disorders, the proportions varied depending on the classification system used (Table 3).

\section{Distribution of dimensions within dsm classification system}

The results were similar for the three diagnostic systems (only DSM classification shown). Between the different DSM diagnostic categories, the median factor scores differed significantly (Kruskal-Wallis test, $p<0.001)$ as did the proportions of individuals scoring above the upper tertile for the different factors (symptom dimensions). For example, in the manic diagnostic category a high proportion scored above the upper tertile on the factor 1 'manic' dimension while individuals diagnosed with depression all scored high on the Factor 3 'depressive dimension'. However, within diagnostic categories there was also considerable within-group variation and overlap between categories (Table 4).

\section{Association of dimensions to known premorbid risk factors}

There was no significant association between any dimension and gender. Age at presentation was significantly associated with disorganisation, depressive and delusional dimensions (adjusted for gender), disorganisation showing the strongest association (estimated regression co-efficient $=-8.50 ; p$-value $<0.00195 \%$ CI $-10.08,-6.91$ ), with the F test of linear restriction showing it to be significantly different from the association between age at presentation and the other dimensions $(p<0.001)$. Disorganisation was also significantly associated with being single at presentation $(\mathrm{OR}=1.35, p=0.002$, $95 \%$ CI 1.11, 1.63), unemployed at onset of symptoms $(\mathrm{OR}=1.35, p=0.02,95 \%$ CI $1.12,1.64)$, having poor premorbid work adjustment $(\mathrm{OR}=1.64, p=0.001$, $95 \%$ CI 1.33, 2.01) and poor premorbid social adjustment $(\mathrm{OR}=1.25, p=0.04,95 \%$ CI 1.01, 1.53), while an inverse relationship was demonstrated with the manic dimension for these risk factors; single $(\mathrm{OR}=0.69, p<0.001,95 \%$ CI $0.56,0.84)$, unemployed $(\mathrm{OR}=0.73, p=0.003,95 \% \mathrm{CI} 0.59,0.90)$ poor premorbid work adjustment $(\mathrm{OR}=0.62, p<0.001$, $95 \%$ CI $0.47,0.81)$ and poor premorbid social adjustment $(\mathrm{OR}=0.53, p<0.001,95 \%$ CI $0.39,0.72)$. No other dimensions were significantly associated with these risk factors except the delusional dimension which was inversely related to poor premorbid social adjustment $(\mathrm{OR}=0.72, p<0.01,95 \%$ CI 0.57 , $0.91)$. Only the depressive dimension was significantly associated with drug /alcohol use within the first year of symptoms $(\mathrm{OR}=1.53, p=0.003,95 \%$ CI 1.15 , $2.01)$ and presence of psychosocial stressors at presentation $(\mathrm{OR}=1.61, p<0.01,95 \% \mathrm{CI} 1.13,2.30)$. It was also associated with family history of other psychiatric disorders $(\mathrm{OR}=1.31, p=0.03,95 \%$ C.I 1.03 , $1.66)$ as was the manic dimension $(\mathrm{OR}=1.30$, $p=0.02,95 \%$ CI 1.04, 1.62). Finally, there were no significant associations between any dimension and a family history of schizophrenia.

Neither the dimensional model (the set of dimensional scores) nor the categorical model (the set of diagnoses) was sufficient to explain the associations 
Table 4 The Proportion of individuals within DSM categories scoring above the upper tertile on symptom dimensions

\begin{tabular}{|c|c|c|c|c|c|}
\hline & $\begin{array}{l}\text { Manic } \\
N(\%)\end{array}$ & $\begin{array}{l}\text { Disorganised } \\
N(\%)\end{array}$ & $\begin{array}{l}\text { Depression } \\
N(\%)\end{array}$ & $\begin{array}{l}\text { Delusional } \\
N(\%)\end{array}$ & $\begin{array}{l}\text { Hallucinations } \\
N(\%)\end{array}$ \\
\hline Schizophrenia & 21 (19) & $57(50)$ & $26(23)$ & $25(22)$ & $39(35)$ \\
\hline Schizophreniform & $21(24)$ & 42 (47) & $32(36)$ & $23(26)$ & $42(47)$ \\
\hline Manic & $62(93)$ & $16(24)$ & $17(67)$ & $47(70)$ & $22(33)$ \\
\hline Major depression & $3(30)$ & $1(10)$ & $10(100)$ & $5(50)$ & $3(30)$ \\
\hline Delusional disorder & $1(3)$ & 0 & 4 (13) & 4 (13) & $1(3)$ \\
\hline Unspecified & $44(31)$ & $38(27)$ & $38(27)$ & $38(27)$ & $44(31)$ \\
\hline
\end{tabular}

(11 cases, which were unclassified and 2 schizoaffective cases, were excluded from this analysis)

Table 5 Contribution of dimensional and categorical models in the associations with premorbid risk factors

\begin{tabular}{|c|c|c|c|c|}
\hline \multirow{2}{*}{$\begin{array}{l}\text { Dependent variable in logistic/linear model } \\
\text { Premorbid risk factor }\end{array}$} & \multicolumn{2}{|c|}{$\begin{array}{l}\text { Comparison of full model with model } \\
\text { constrained by dropping the categorical } \\
\text { diagnoses }\end{array}$} & \multicolumn{2}{|c|}{$\begin{array}{l}\text { Comparison of full model with model } \\
\text { constrained by dropping the dimensions }\end{array}$} \\
\hline & Likelihood ratio statistic & $p$-value & Likelihood ratio statistic & $p$-value \\
\hline Gender & 10.68 & 0.06 & 4.44 & 0.49 \\
\hline Age at presentation & 22.78 & $<0.001$ & 86.14 & $<0.001$ \\
\hline Single at presentation & 8.20 & 0.15 & 15.38 & $<0.01$ \\
\hline Unemployed at presentation & 13.63 & 0.02 & 10.25 & 0.07 \\
\hline Poor work adjustment & 15.61 & $<0.01$ & 15.35 & $<0.001$ \\
\hline Poor social adjustment & 19.25 & $<0.001$ & 2.59 & 0.76 \\
\hline Alcohol/drugs misuse & 6.06 & 0.19 & 18.92 & 0.002 \\
\hline Psychosocial stressor & 12.90 & 0.02 & 18.07 & 0.002 \\
\hline Family History schizophrenia & 6.25 & 0.28 & 6.37 & 0.27 \\
\hline Family psychiatric history & 1.84 & 0.76 & 8.67 & 0.12 \\
\hline
\end{tabular}

with all the pre-morbid variables (Table 5). None of the posited models were significantly related to gender, family history of schizophrenia or family history of other psychiatric disorders. The set of dimensions was an adequate model of association for single at presentation and alcohol/drug misuse within the first year of onset. However, the categorical model was more informative for poor social adjustment and unemployment at presentation. The full model (both sets of data) best represented the association of age at presentation, poor work adjustment and presence of a psychosocial stressor i.e., neither the dimensional or categorical model was sufficient on their own.

\section{Discussion}

\section{Findings}

In a population-based study of patients during the first year of a psychotic disorder we have shown a symptom structure composed of 5 dimensions; 'mania', 'disorganisation/bizarre', 'depression', 'nonbizarre/non-mood congruent delusions' and 'auditory hallucinations,' which explains $58 \%$ of the total variance. This solution was replicated when EFA were carried out on randomly split halves of the sample. Although diagnostic categories had different symp- tom dimension profiles, these did not relate precisely to the diagnoses of psychoses in the currently used classification systems. Symptom dimensions showed different and distinct profiles of associations with known pre-morbid risk factors i.e., individual dimensions were able to discriminate between known risk factors. Our results indicate that using both the dimensional and categorical models of psychosis was more informative in terms of sensitivity to variation in demographic and risk factor profiles than either model independently.

\section{Methodological considerations}

Before interpreting our findings, we must consider potential limitations. Symptoms and risk factors were identified via case note review, although this is not the best way to detail clinical variables, the case notes were comprehensive and well maintained. The 2 raters were experienced psychiatrists demonstrating good inter-rater reliability for both individual item ratings and diagnostic categories. We used OPCRIT, to cover a wide range of psychopathology and demographic information, however not every symptom or known risk factor with a theoretical relationship to psychosis could be included, OPCRIT does not have a broad range of anxiety, catatonic or negative symptoms. A few candidate items had low variability, 
which could reflect the chart review method, with clinicians perhaps failing to record all symptoms, however studies using face-to-face interviews have found similarly low prevalence for items such as insight [11]. Finally, we created composite items where symptoms showed high collinearity. With regard to pre-morbid functioning we used overall measures, however better discrimination may have been achieved if information on different domains of these validators were available [27]. The EFA solution has moderate communalities (average 0.59 ) and moderate over-determination of factors (one factor only having 2 items), however the large sample size should be adequate to produce accurate estimations under these conditions [14] replication of the structure using split half samples strengthens the EFA findings. Finally, multiple statistical tests were conducted to explore the relationship between dimensions and demographic risk factors this could theoretically result in a Type 1 error, however no adjustment for multiple tests have been used, as the a priori hypothesis was that differential associations would be present and adjustment could potentially result in important differences being deemed non-significant [32].

\section{Symptom dimensions}

Affective symptoms loaded on to two distinct 'manic' and 'depression' domains. This is consistent with findings from previous studies of psychoses rated using OPCRIT [25, 30, 36, 37] and in studies of recent onset psychosis. [23, 28]. The negative symptom blunted/flattened affect loaded on to this dimension in the opposite orientation to the manic symptoms, similar to another recent study [23].

The second 'disorganisation' factor' is also supported in the literature [17]. However, this has generally been associated with a negative domain, which we did not identify separately. It is possible that this is due to OPCRIT taking a narrow view of negative symptoms, focusing on negative formal thought disorder, restricted affect and blunted affect. This narrow view is, however stressed in the literature as it increases the chance of only primary negative symptoms being elicited rather than those secondary to positive symptoms or medication [10]. We excluded negative formal thought disorder in this study as it had a low prevalence, which in part may be consequent on the case note review methodology, as clinician's tend to document only prominent and severe poverty of speech. However, previous studies have found the symptoms of 'blunting of affect' and 'inappropriate affect' (both present in our study) to best discriminate between negative and disorganised syndromes while 'negative formal thought disorder' was a poor discriminator $[5,41]$. Also, previous studies using OPCRIT have identified negative factors within mixed stage/chronic samples, where negative symptoms have a higher prevalence $[6,30]$. In order to examine the issue of negative symptoms in relation to the disorganisation factor, a post-hoc analysis was carried out in which associations between the dichotomous measures of negative symptoms (blunting/restricted affect) and, jointly, the five dimensions were assessed. This revealed significant associations with all five dimensions, but the odds ratio was strongest by far for the disorganisation dimension $(\mathrm{OR}=7.5,95 \% \mathrm{CI} 4.2,13.1)$ and significantly stronger than the OR with the depressive dimension $\left(\chi^{2}=5.3\right.$, $p=0.02)$, the hallucinations dimension $\left(\chi^{2}=32.3\right.$, $p<0.0001)$, the mania dimension $\left(\chi^{2}=42.8\right.$, $p<0.0001)$ and the delusion dimension $\left(\chi^{2}=36.7\right.$, $p<0.0001)$. Therefore, the disorganisation factor identified in this study to a degree, taps into the negative symptom domain, and with time, the dynamics of these associations may come more to the fore. Apart from the negative symptoms of blunted/restricted affect, we also found bizarre delusions (including schneiderian first rank delusions) to load moderately on to this dimension. In this first episode sample, there seems to be a clinically distinct dimension of disorganisation/bizarre delusions, which includes elements of both Bleulerian and Shneiderian constructs.

Most EFA studies suggest a unitary positive symptom dimension; however, not all studies of recent onset psychosis have found this single factor. $[12,28,43]$. We have found delusions to load on to three factors; bizarre delusions loading with the disorganisation symptoms; mood congruent delusions with the affective dimensions and finally persecutory and systematised delusions loading on a discrete dimension; suggesting potentially different underlying psychopathological mechanisms for different forms of delusion. Auditory hallucinations have loaded on to a separate dimension, this segregation of delusions and hallucinations has been reported previously $[26,41]$.

\section{Relationship of symptom dimensions to clinical diagnostic categories}

Diagnostic categories differed in their factor score profiles however; there was considerable overlap between categories. For example, disorganisation/bizarre is more common in DSM-IV schizophrenia (50\%), but was not uncommon in other forms of psychosis. For example, 25\% of patients diagnosed with mania scored in its top tertile. Similarly, $24 \%$ of the subjects diagnosed with DSM-IV schizophrenia scored in the highest tertile for the mania dimensional scores. The range of scores in each diagnostic category also indicates considerable intra-group variation. The mildly correlated factor solution and the observed overlap between categories on their factor score profiles suggests that symptom dimensions do not define classes of patients but co-exist within individuals. 


\section{The association of dimensions and categories to known pre-morbid risk factors}

There was a differential association between dimensions and risk factors e.g., the disorganised factor was strongly associated with younger age of onset, single status, unemployment and poor pre-morbid work and social adjustment. On the other hand, increasing scores in the manic dimension increased the probability of being married, employed and having good pre-morbid work and social adjustments. Manic and depressive dimensions were both associated with family history of psychiatric disorders (other than schizophrenia). Previous studies exploring the relationship between symptom dimensions and a family history of psychosis have been inconsistent showing either no or only weak associations. [7, 25, 29]. We did not find an association between any of the dimensions (or diagnoses) and a family history of schizophrenia, this result is consistent with findings from family studies, showing family history of nonaffective psychosis throughout the spectrum of affective and non-affective psychosis [20].

Finally, neither dimensional or categorical models alone could best predict associations with pre-morbid risk factors, rather concomitant use of both representations provided significantly more information than either one system independently. The concurrent use of alternative representations of psychopathology has been proposed [40] and our finds support this assertion.

This dimensional model of first episode psychosis requires further validation using neurobiological strategies and prognostic variables nonetheless the results presented here support the idea that it is useful to conceive psychosis as overlapping symptom dimensions, each associated with a range of underlying and different risk factor profiles. However, in this study, the concomitant use of both dimensional and categorical models of psychosis was more sensitive to variations in demographic and risk factors than either model independently; combining dimensional and categorical representations of psychosis is likely to be most informative in elucidating the causes and correlates of psychosis.

Aknowledgements Thanks to Dr David Young, Department of Statistics \& Modelling Science, Strathclyde University for statistical advice. This work was supported by a grant from The Stanley Foundation.

\section{References}

1. Allardyce J, Morrison G, Van Os J, Kelly J, Murray RM, McCreadie RG (2000) Schizophrenia is not disappearing in south-west Scotland. Br J Psychiatry 177:38-41

2. Altman DG (1991) Practical statistics for medical research. Chapman and Hall, London
3. American Psychiatric Association (1994) Diagnosistic and statistical manual of mental disorders (DSM-IV), 4th edn. APA, Washington, DC

4. Azevedo MH, et al. (1999) Using consensus OPCRIT diagnoses. Br J Psychiatry 175:154-157

5. Brown K, White T (1992) Syndromes of schizophrenia and some clinical correlates. Br J Psychiatry 161:317-322

6. Cardno AG, et al. (1996) Factor analysis of schizophrenic symptoms using the OPCRIT checklist. Schizophr Res 22:233239

7. Cardno AG, Holmans PA, Harvey I, Williams MB, Owen MJ, McGuffin P (1997) Factor-derived subsyndromes of schizophrenia and familial morbid risks. Schizophr Res 23:231-238

8. Cattell RB (1966) The scree plot for the number of factors. Multivariate Bahav Res 1:245-276

9. Craddock N, Asherson P, Owen MJ, Williams J, McGuffin P, Farmer A (1996) A concurrent validity of the OPCRIT diagnoses with concensus best-estimate lifetime diagnoses. Br J Psychiatry 169:58-63

10. Crow TJ (1985) The two-syndrome concept: origins and current status. Schizophr Bull 11:471-486

11. Crumlish N, et al. (2005) Early insight predicts depression and attempted suicide after 4 years in first-episode schizophrenia and schizophreniform disorder. Acta Psychiatr Scan 112:449455

12. Drake RJ, Dunn G, Tarrier N, Haddock G, Haley C, Lewis S (2003) The evolution of symptoms in the early course of nonaffective psychosis. Schizophr Res 63:171-179

13. Eaton WW, Thara R, Federman B, Melton B, Liang KY (1995) Structure and course of positive and negative symptoms in schizophrenia. Arch Gen Psychiatry 52:127-134

14. Fabrigar LR (1999) Evaluating the use of exploratory factor analysis in psychological research. Psychol Methods 4:272299

15. Fenton WS, McGlashan TH (1991) Natural history of schizophrenia subtypes. II. Positive and negative symptoms and longterm course. Arch Gen Psychiatry 48(11):978-986

16. Gorsuch RL (1983) Factor analysis. Laurence Erlbaum Assocites, New Jersey

17. Grube BS, Bilder RM, Goldman RS (1998) Meta-analysis of symptom factors in schizophrenia. Schizophr Res 31:113-120

18. Hosmer DW, Lemeshow S (2000) Applied logistic regression. Wiley, New York

19. Kendell R, Jablensky A (2003) Distingusihing between the validity and the utility of psychiatric diagnoses. Am J Psychiatry160:4-12

20. Kendler KD, McGuire M, Gruenberg AM, Spellman M, O’Hare A, Walsh D (1993) The roscommon family study II. The risk of nonschizophrenic nonaffective pshoses in relatives. Arch Gen Psychiatry 50:645-652

21. Matsuura $\mathrm{M}$, et al. (2004) A polydiagnostic and dimensional comparison of epileptic psychoses and schizophrenia spectrum disorders. Schizophr Res 69:189-201

22. Maziade M, Roy MA, Martinez M, Cliché D (1995) Negative, psychoticism, and disorganized dimensions in patients with familial schizophrenia or bipolar disorder: continuity and discontinuity between the major psychoses. Am J Psychiatry 152:1458-1463

23. McGorry PD, Bell RC, Dudgeon PL, Jackson HJ (1998) The dimensional structure of first episode psychosis: an exploratory factor analysis. Psychol Med 28:935-947

24. McGuffin P, Farmer A, Harvey I (1991) A polydiagnostic application of operational criteria in studies of psychotic illness. development and reliability of the OPCRIT system. Arch Gen Psychiatry 48:764-770

25. McIntosh AM, et al. (2001) A factor model of the functional psychoses and the relationship of factors to clinical variables and brain morphology. Psychol Med 31:159-171

26. Mellers JD, Sham P, Jones PB, Toone BK, Murray RM (1996) A factor analytic study of symptoms in acute schizophrenia. Acta Psychiatr Scan 93:92-98 
27. Norman RMG, Malla AK, Manchanda R, Townsend L (2005) Social Academic premorbid adjustment. Acta Psychiatr Scan 112:30-39

28. van Os J, et al. (1996) Psychopathological syndromes in the functional psychoses: associations with course and outcome. Psychol Med 26:161-176

29. van Os J, Marcelis M, Sham P, Jones P, Gilvarry K, Murray R (1997) Psychopathological syndromes and familial morbid risk of psychosis. Br J Psychiatry 170:241-246

30. van Os J, et al. (1999) A comparison of the utility of dimensional and categorical representations of psychosis. UK700 Group. Psychol Med 29:595-606

31. Peralta V, Cuesta MJ (2001) How many and which are the psychopathological dimensions in schizophrenia? Issues influencing their ascertainment. [Review] [68 refs]. Schizophr Res 49(3):269-285

32. Perneger TV (1998) What's wrong with Bonferroni adjustments. Br Med J 316:1236-1238

33. Ratakonda S, Gorman JM, Yale SA, Amador XF (1998) Characterization of psychotic conditions. Use of the domains of psychopathology model.[see comment]. Arch Gen Psychiatry 55:75-81

34. Rosenman S, Korten A, Medway J, Evans M (2003) Dimensional vs. categorical diagnosis in psychosis. Acta Psychiatr Scan 107:378-384

35. Serretti A, Lattuada E, Cusin C, Smeraldi E (1999) Factor analysis of delusional disorder symptomatology. Compr Psychiat 40(2):143-147

36. Serretti A, et al. (2001) Major psychoses symptomatology: factor analysis of 2241 psychotic subjects. Eur Arch Psychiatry Clin Neurosci 251:193-198
37. Serretti A, Olgiati P (2004) Dimensions of major psychoses: a confirmatory factor analysis of six competing models. Psychiatry Res 127:101-109

38. Spitzer R, Gibbon M, Endicott J (1975) Mental status schedule. Arch Gen Psychiatry 35:773-782

39. StataCorp. Stata statistical software. Release 9. 2005. College Station, Texas

40. Stordal KI, et al. (2005) General psychopathology is more important for executive functioning than diagnosis. Acta Psychiatr Scan 111:22-28

41. Thompson PA, Meltzer HY (1993) Positive, negative, and disorganisation factors from the schedule for affective disorders and schizophrenia and the present state examination. A threefactor solution. Br J Psychiatry 163:344-351

42. Toomey R, et al. (1997) Revisiting the factor structure for positive and negative symptoms: evidence from a large heterogeneous group of psychiatric patients. Am J Psychiatry 154:371-377

43. Vazquez-Barquero JL, Lastra I, Nunez MJC, Herrera CS, Dunn G (1996) Patterns of positive and negative symptoms in first episode schizophrenia. Br J Psychiatry 168:693-701

44. Williams J, Farmer AE, Achenheil M, Kaufmann CA, McGuffin P (1996) A multicentre inter-rater relability study using OPCRIT diagnostic system. Psychol Med 26:775-783

45. Woods R, Wilson D, Gibbons R (2003) TESTFACT in IRT from Mathida du Toit. In: Scilling S, Muraki E, Bock D (eds) Lincolnwood, Scientific Software International. INC

46. World Health Organisation (1992) The tenth revision of the international classification of diseases amd related health problems (ICD-10). WHO, Geneva 

CHAPTER 10

Diagnostic Utility: categories and dimensional representations of psychosis comparison of their discriminate validity with 5 -year outcome

Allardyce, J. \& van Os, J. on behalf of the D\&G 464 study group submitted 


\section{ABSTRACT}

Background \& Aims: The American Psychiatric Association's Diagnostic and Statistical Manual of Mental Disorders (DSM) is under revision and the inclusion of a dimensional measure of symptoms to improve clinical usefulness is being considered, however, the predictive power of symptom dimensions is not yet established. Few studies have examined dimensional relationships with outcome and there are currently, no published follow-up studies of dimensions generated from first- episode psychosis samples. This study adds to the current literature by examining the usefulness of symptom dimensions and DSM IV categorical diagnoses (derived from incident cases of psychosis) in predicting 5-year outcome. Methods: From 379 incident cases of psychosis, outcome, measured using individual descriptors of symptom course/disability and 'classes of outcome' (generated using finite mixture modeling)- from longitudinal information covering the initial five years from presentation, were regressed onto 3 competing explanatory models i) complementary model using diagnoses and dimensions ii) diagnoses only iii) dimensions only. Results: The explanatory models using information from both dimensions and diagnoses had better predictive power. For the 3 outcome classes the complementary model (dimensions and diagnoses) had a pseudo R-squared of 0.24 , while the model with factor scores only had a pseudo R-squared 0.16 and diagnoses only model a pseudo R-squared of 0.19 . This difference was statistically significant, Likelihood ratio test (LRT) statistic for the dimensions only model $=36.26, \mathrm{p}$ value $<0.001$ and Diagnoses only model LRT $=63.13, \mathrm{p}$ value <0.001).Conclusion: The complementary use of DSM IV diagnoses and dimensional (factor) scores, adds significantly to the predicative power of the DSM diagnoses. Diagnoses seem to be more useful than dimensions around the time of presentation, while dimensional representations may provide added information at follow up. Keywords: schizophrenia, psychosis, classification, diagnoses, dimensions. 


\section{INTRODUCTION}

Despite significant advances in our knowledge of the neuroscience underpinning mental disorders, substantial gaps in genetic and pathophysiological understandings remain, which hinder the development of an aetiological-based classification system, corroborated by specific biological markers ${ }^{1}$. Consequently, outcome remains an important criterion for validating psychiatric diagnoses ${ }^{2-5}$. Valid, useful diagnoses will have good predictive power and provide clinically relevant information about course, outcome and likely treatment response.

The American Psychiatric Association's Diagnostic and Statistical Manual of Mental Disorders (DSM) is currently under revision and the possibility of introducing a dimensional or qualitative component to improve clinical usefulness, is under consideration, 6 however the predictive power of symptom dimensions is not yet established. Few studies have examined dimensional relationships with course and outcome and to date, there are no published follow-up studies of dimensions generated from first- episode psychosis samples.

We therefore report a study comparing the prognostic usefulness of symptom dimensions and DSM IV categorical diagnoses, derived from incident cases of psychosis. Outcome is a multidimensional construct, comprising inter-linked domains relating to; symptom course, longitudinal social disability and treatment $7-10$. In order to take account of these associations, results are presented for outcome measured by i) individual descriptors of course and outcome and ii) classes (typologies) of outcome generated using finite mixture modeling (a multivariate reduction technique) - from longitudinal information covering the initial five years from presentation.

\section{METHODS}

\section{Catchment area}

Dumfries and Galloway (D\&G) is a geographically well-defined area in South-West Scotland. It has a stable population of around 147,000, of whom $99.5 \%$ are white. Psychiatric services are via one hospital and its associated community services. There is little or no private health care.

\section{Baseline patient identification \& characteristics}

Case ascertainment has been described in detail elsewhere ${ }^{11}$. Briefly, all patients were identified who came into contact (in-patients, day patients, out patients, domiciliary assessments and informal out-of-hour contacts) with psychiatric services in Dumfries and Galloway over a twentyyear period (1979-98) and who were given a clinical diagnosis of schizophrenia, schizoaffective disorder, delusional disorder, mania, drug induced psychotic disorder; acute, transient or unspecified psychotic disorder. Patients who were not residents of Dumfries \& Galloway, who had experienced psychotic symptoms prior to the study period or had a clear organic cause for their symptoms, were excluded. 464 patients had a first episode of psychosis during the study period. There were 210 (45.26\%) males and 254 (54.74\%) females with a median age at presentation of 36 (inter-quartile range 25-55). Males and females showed a significant difference in the age of onset, the median age for males was 33 and for females it was 39.5 years $(p<0.001)$ 


\section{Baseline assessment of psychopathology}

Psychopathological assessment has been described in detail previously ${ }^{12-14}$. Briefly, two experienced psychiatrists, working independently, used the OPCRIT checklist 15 to rate the presence and absence of symptoms from the case records (which included medical, informant, nursing, social work, occupational therapy notes and all correspondence) for the first year from presentation. Good inter-rater agreement was demonstrated for both the individual symptom measures (kappa ranged $=0.69-0.92$ ) and DSM IV diagnoses (kappa $=0.79$ ) generated using the associated diagnostic algorithm OPCRIT 3.4.

A exploratory principal factor analysis on the tetrachoric correlation matrix with promax rotation of first year OPCRIT ratings was carried out and identified five latent dimensions (mildly correlated), explaining $58 \%$ of the variance. Affective symptoms loaded on to two distinct 'manic' and 'depression' factors. A third factor predominately consisted of disorganised symptoms, which also included moderately loaded negative symptoms and a fourth, positive symptom factor. Delusions loaded on to three factors; bizarre delusions with the disorganisation symptoms; mood congruent delusions with the affective dimensions but widespread systematised delusions loading on a discrete fifth dimension. This factor structure was replicable, with the solutions from random split-halves of the sample being highly correlated. The four-factors manic, depressive, positive and disorganised/negative are consistent with factor analytical studies of recent onset psychosis ${ }^{16}$; a separate delusional dimension has similarly been reported in other studies using instruments allowing for multiple measures of delusional ideation ${ }^{17,18}$. Factor scores were calculated for each individual for the five dimensions ${ }^{14}$

\section{Tracing exercise}

The Local Regional Ethics Committee and the Privacy Committee of Information and Statistical Division (ISD) Scotland approved the follow up study. A Careful tracing exercised was carriedout; over a 2-year period (2004-2005), using the D\&G medical records department; the General Register Office, Edinburgh (for births, deaths and marriages); the National Health Service (Scotland) Central Registrar and the current electoral role for Scottish wards. 422 (91\%) of the inception cohort were traced, $133(32 \%)$ had died and the median time from presentation was 11.82 years (inter quartile range 6.67-18.41 years). Once traced, subjects each received a letter introducing the study, followed by a visit from a research worker who explained the purpose of the study and asked for their consent.

\section{Clinical outcome \& social disability assessment}

Where possible, multiple sources of information including patient interviews, careful systematic review of case notes and supplementary information from health professionals involved in dayto-day patient care and family members were used in order to complete a lengthy assessment battery. For the purpose of the analyses presented in this paper, ratings were used for the first 5 years of follow-up, from two sources. First, a slightly modified version of the WHO Life Chart Schedule (LCS) 19, 20 which rates outcome across four domains of i) overall psychotic symptom duration, severity and periodicity coded by a single rater (JA), ii) treatment, iii) residency, iv) purposeful activity domains, rated by research workers demonstrating good reliability (pairwise agreement $>0.8$ for all items used in the analyses). Second, The Life Time Dimensions Scale (LTDS) ${ }^{21}$ was used to rate individual items of psychopathology longitudinally and multidimensionally, coded by a single rater (JA). 


\section{Multivariate reduction of outcomes across domains}

Mixture Modelling using Mplus version $4{ }^{22}$ was carried out to identify subtypes of patients with shared outcome profiles (latent classes). This multivariate analysis was applied to 34 indicators of outcome from the standardised schedules, LCS and LTDS, chosen (a priori) to represent the broad range of outcomes (items presented in TABLE 2). The aim of this analysis was to identify the smallest number of classes, which adequately describe the observed associations seen in the outcomes, starting with the most parsimonious 1 class model and fitting successive models with increasing numbers of classes. As the LCA models with different number of classes are not nested, it is inappropriate to use chi squared differences to compare the fit of 2 competing models when determining the optimal solution. Therefore, the number of classes was informed by goodness of fit indices; the Akaike information criterion (AIC) and Bayesian information criterion (BIC) (where lower values indicate better fit). As the response pattern of the outcome measures was large, it is unlikely that the likelihood ratio statistic would follow the theoretical chi squared distribution; therefore, we present boot strapped and Lo-Mendell-Rubin likelihood ratio test (LRT) p-values when comparing models. The Local independence assumption was checked by examining the bi-variate residuals and finally, standardised entropy scores were used to measure classification performance (higher scores indicating better classification performance).

\section{Comparison psychopathology-derived predictive models for outcome}

A series of linear/logistic/ordinal logistic regression models were fitted for each outcome measure. First, a "full model" was generated for each outcome (as dependent/response variable) and regressed onto both the factor scores and diagnoses (DSM IV) (independent/ predictive variables). In order to assess the relative contribution of dimensions and diagnoses, the explanatory power of this full model was compared to similar models constrained by dropping the diagnoses and the dimensional components in turn. Statistical significance of model differences was estimated using the likelihood ratio test.

\section{RESULTS}

\section{Attrition at 5 years}

High quality information pertaining to the first 5 years of follow up was obtained for 379 (82\%) of the total inception cohort. Of these, 351 (93\%) had continued contact with psychiatric services (at 5 years) and 127 (36\%) had a face-to-face interview. 85 (18\%) of the original cohort were lost at 5 years due to $56(12 \%)$ having died and $29(6 \%)$ having inadequate information.

\section{Comparison followed up / lossed to follow up (Itfu)}

There was no statistically significant difference in distribution of gender, year of presentation, pre-morbid social adjustment, alcohol use in the first year, cannabis use in the first year or index diagnosis between the two groups. However, those followed up were more likely to have younger age of onset (median 33 years compared to 58.5 in LFTU; $t=7.4, p<0.001)$, be single $\left(\chi^{2}\right.$ $=4.49, \mathrm{p}=0.03)$, unemployed $\left(\chi^{2}=4.45, \mathrm{p}=0.03\right)$, have a family history of schizophrenia $\left(\chi^{2}=\right.$ 
4.42, $\mathrm{p}=0.03)$ and family history of other psychiatric disorders $\left(\chi^{2}=8.09, \mathrm{p}=0.04\right)$. This bias reflects the primary cause of attrition, which was death.

\section{Multivariate reduction of outcome measures}

Investigating fit measures for 1 to 4 class models, showed the BIC and AIC to dip at the 3 class solution and this was corroborated by the Lo- Mendell- Rubin LRTs becoming non significant, when comparing the 3 and 4 class model, though the boot strapped LRT did suggest a higher number of classes. Entropy was high for all models (Table 1). Overall, the most parsimonious model was the 3-class model which seems to be an adequate descriptor of observed variability of outcome and the bivariate residuals were respectable. The conditional probabilities and average class means of outcome indicators appear in Table 2. Class 1 (31\% of the follow up cohort) tended to exhibit pervasive disability with longest time psychotic (mean 59.56 months, sd 0.32) and $35 \%$ probability of experiencing severe negative symptoms for more than 2 years. They required the highest levels of residential support (mean 18.88 months, sd 2.29) and unemployment (mean 33.22 months, sd 2.55) and had a high probability of global deterioration in functioning (0.65). Class 3 (23\% of the cohort) showed the best outcome with a mean time psychotic of 2.46 months (sd 0.25 ), high probability of experiencing complete remission $(0.63)$ and low likelihood of significant deterioration in functioning (0.17), with on average the shortest time in supported residence (mean 14.30 months, sd 1.82) and lowest unemployment (mean 10.05 months, sd 2.28). Class 2 (46\% of the cohort) exhibited intermediate duration of symptoms and disability. There is a monotonic change in the conditional probabilities (or class means) of indicators across classes for all items, with the exception of 2 severity scores, demonstrating increasing severity of outcome i.e. ordinal classes.

TABLE 1: Fit Indices for mixture modelling (LCA) of psychosis outcome

\begin{tabular}{lllllll} 
Model & Log likelihood & BIC & AIC & Lo-Mendell-Rubin LRT & $\begin{array}{l}\text { Bootstrap } \\
\text { LRT }\end{array}$ & entropy \\
& & & & & - & \\
1 class & -10174 & 20557 & 20419 & - & $<0.001$ & 1 \\
2 class & -9006 & 18414 & 18149 & 0.32 & $<0.001$ & 0.97 \\
3 class & -8745 & 18090 & 17692 & 0.05 & $<0.001$ & 0.99 \\
\hline class & -8582 & 17959 & 17431 & $\sim 1$ & \\
\hline
\end{tabular}


TABLE 2: Class Solution of indicators of 5 year outcome of psychosis

Latent class conditional probabilities (or estimated means) for 3 class

solution for outcome at 5 years

\section{CLASSES}

PREVALENCE

outcome

118

(31\%)

Intermediate

Best out-

59.56

Time of longest psychotic episode (LCS)

(sd 0.32) outcome

come

175

86

$(46 \%)$

$(23 \%)$

Time requiring residential support (LCS)

18.88

5.03

2.46

(sd 2.29)

(sd 0.42)

(sd 0.25)

14.30

13.23

33.22

(sd 1.82)

(sd 2.58)

(sd 2.55)

23.97

10.05

Time unemployed or not engaged in purposeful activity (LCS)

0.65

(sd 2.27)

(sd 2.28)

Over all deterioration within 5 years (LCS)

0.36

0.17

Duration of delusions (LTDS)

Present for delusions for days but $<2$ weeks

0.01

0.12

0.32

0.30

Present for delusions $>2$ months to $<2$ years

0.12

0.61

0.21

Present for delusions $>=2$ years

0.03

0.04

0.00

Presence of paranoia for days but $<2$ weeks

Presence of paranoia $>2$ months to $<2$ years

Presence of paranoia $>=2$ years

0.06

0.20

0.10

0.75

0.42

0.01

Duration of any hallucinations (LTDS)

Present for any hallucinations for days but $<2$ weeks

0.11

0.34

0.00

Presence of any hallucination $>2$ months to $<2$ years

Presence of any hallucination $>=2$ years

0.14

0.32

0.19

0.41

0.27

0.11

Duration of formal thought disorder (FTD) (LTDS)

Present of FTD for days but $<2$ weeks

0.10

0.01

0.00

Presence of FTD $>2$ months to $<2$ years

0.13

0.12

0.07

Presence of FTD $>=2$ years

0.12

0.13

0.00

Severity of delusions (LTDS)

Rated moderate and clinically relevant

0.61

0.01

0.00

Rated severe or very severe

0.25

0.36

0.20

Severity of Paranoid Ideation (LTDS)

Rated moderate and clinically relevant

0.61

0.31

Rated severe or very severe

0.59

0.10

0.22

0.38

0.02

Severity of any hallucinations (LTDS)

Rated moderate and clinically relevant

0.55

0.35

0.21

Rated severe or very severe

0.10

0.26

0.09

Severity of Formal Thought Disorder (LTDS)

0.22

0.15

0.07

Rated severe or very severe

0.11

0.00

Presence of any Sneiderian first rank symptom, for at least 2 months

Rated severe or very severe

0.17

0.31

0.07

Presence of negative symptoms (blunted affect or poverty of speech) for more than 2 years (LTDS)

Rated moderate and clinically relevant

Rated severe or very severe

0.15

0.35

0.08

0.00

Type of remission (LCS)

None

Incomplete (residual symptoms remain)

Complete (no residual symptoms evident)

0.03

0.43

0.32

Characteristics of course (LCS)

Sing episode

0.01

0.60

0.63

Multiple episodes

0.40

0.57

0.03

0.59

0.43

Chronic symptoms

0.97

0.00 


\section{COMPARISON OF PSYCHOPATHOLOGY-DERIVED EXPLANATORY MODELS OF OUTCOME}

The results comparing the predicative power of i) full model using both diagnoses and factor scores (symptom dimension scores) ii) factor scores only and iii) diagnoses only, are presented in (Table 3). Diagnoses explained an important (statistically significant) proportion of the full predictive model for all individual outcome measures. However, the dimensional representations added significantly to the explanatory model for time psychotic, single episode, chronic course, type of remission. The explanatory models using the outcome classes found the full model (dimensions and diagnoses) to have pseudo McFadden's R-squared values of 0.24 , while the model with factor scores only had a pseudo McFadden's R-squared was 0.16 and diagnoses only model a McFadden's pseudo R-squared of 0.19. However, both individual models significantly contributed to the explanatory power of the full model (LRT statistic for: dimensions only model $=36.26$, $\mathrm{p}$ value $<0.001$, Diagnoses only model $=63.13$, $\mathrm{p}$ value $<0.001$ ) .

TABLE 3: Comparison of psychopathology derived predictive models with outcome

\begin{tabular}{|c|c|c|c|c|c|c|c|}
\hline \multirow{2}{*}{$\begin{array}{l}\text { Outcome } \\
\text { Time psychotic }\end{array}$} & \multirow{2}{*}{$\begin{array}{l}\mathrm{R}^{2} \\
\text { Full model } \\
0.27\end{array}$} & \multirow{2}{*}{$\begin{array}{l}\mathrm{R}^{2} \\
\text { For dimen- } \\
\text { sional model } \\
0.15\end{array}$} & \multicolumn{2}{|c|}{$\begin{array}{l}\text { LRT } \\
\text { Difference full model } \\
\text { with } \\
\text { dimensional model } \\
\text { (dropping diagnoses) }\end{array}$} & \multirow{2}{*}{$\begin{array}{l}\mathrm{R}^{2} \\
\text { For diagnoses } \\
\text { model } \\
0.23\end{array}$} & \multicolumn{2}{|c|}{$\begin{array}{l}\text { LRT } \\
\text { Difference full model } \\
\text { with } \\
\text { Diagnoses model } \\
\text { (dropping dimensions) }\end{array}$} \\
\hline & & & 25.72 & $<0.001$ & & 59.20 & $<0.001$ \\
\hline Functional Deterioration & 0.10 & 0.07 & 3.02 & 0.699 & 0.09 & 14.91 & 0.011 \\
\hline Single episode & 0.10 & 0.06 & 11.79 & 0.041 & 0.07 & 18.73 & 0.002 \\
\hline Chronic course & 0.23 & 0.14 & 23.96 & $<0.001$ & 0.18 & 39.72 & $<0.001$ \\
\hline Type of remission & 0.15 & 0.08 & 36.41 & $<0.001$ & 0.10 & 52.27 & $<0.001$ \\
\hline $\begin{array}{l}\text { Time requiring residen- } \\
\text { tial support }\end{array}$ & 0.14 & 0.10 & 5.13 & 0.401 & 0.11 & 17.95 & 0.003 \\
\hline Time unemployed & 0.22 & 0.16 & 2.12 & 0.835 & 0.20 & 17.17 & 0.004 \\
\hline
\end{tabular}

\section{DISCUSSION}

\section{Best psychopathology-derived predictive model for outcome}

Using both DSM IV diagnoses and symptom dimensions concurrently predicted 5-year outcome of psychosis significantly better than either diagnosis or dimensional representation on their own, in this (population-based) follow up of patients with first episode psychosis. DSM categorical diagnosis independently explained an estimated $19 \%$ of the total variance of the overall measure of outcome, suggesting that the current diagnoses are relatively robust predictors of outcome. However factors scores (symptom dimensions) added significantly to their predictive power, with the combined model explaining $24 \%$ of variability of outcome. Thus, dimensions seem to increase the predictive power of DSM diagnoses. 
Although there have been many studies examining the factor structure of psychosis ${ }^{23}$, few have examined their association with outcome and disability 24-31, and even fewer comparing the utility of dimensions and diagnoses 24, 29-31 (summarized in Table 4). Generally, these studies report dimensions to be more useful than categories in predicting service use and social disability though the difference in the competing models predictive power is small and not demonstrated across all outcomes. For example, categorical representation have been shown to better predict illness duration ${ }^{27}$, use of support services and 'deteriorating course' ${ }^{29}$, while other studies suggest that neither dimensional or categorical models alone can best predict associations with outcomes such as level of impairment during episodes, deterioration in functioning, or response to neuroleptic treatment 24 . The lack of consistency in results may in part be consequence of the study design. Firstly, they study psychopathological representations generated from samples, which vary by 'stage of disorder'. However, models of underlying psychopathological structure, derived from latent variable statistical models (e.g. exploratory factor analyses or latent class analyses) are highly dependent on when in the evolution of the disorder the symptoms are assessed 27,32 . To overcome this problem, patients were studied using psychopathology measured during the first year from presentation, as this is an established validating criterion for nosological classification ${ }^{5}$. This methodological difference may explain in part our findings that both dimensions and diagnoses predict outcome over and above the other and categories have greater predictive power than dimensions. In previous studies, where lifetime ratings of psychopathology were used, there will be accretion of symptomatic information over time and it is quite possible that dimensional scaling utilizes this cumulative psychopathology more effectively. Secondly, few of the previous studies have used formal standardised assessment tools designed specifically for the measurement of course and outcome, we therefore choose to measure course and disability with the LCS, a schedule which has been used extensively in outcome studies and has proven reliability ${ }^{33}$. This was supplemented with information from the LTDS, a scale specially designed to rate long-term symptomatic information from retrospective review of case notes and has proven reliability ${ }^{21}$. In this study, one experienced clinician rated the symptoms over 5 years, obviating the issue of inter-rater reliability, although problems could still occur if the single rater scored everything erroneously. However, even if this were the case, there would still be no reason to suspect differential measurement error across dimensions or diagnoses assigned at baseline. In addition, previous papers have reported multiple individual indicators of outcome, with multiple statistical testing that could potentially lead to chance findings. We dealt with this problem by using multivariate techniques to reduce outcome into discrete classes. 
TABLE 4: Summary of published studies of the comparative utility of diagnoses and dimensions in predicting outcome.

\begin{tabular}{|c|c|c|c|c|c|}
\hline Study & Outcome measure & $\begin{array}{l}\text { Estimation of predic- } \\
\text { tive power } \\
\text { Dimensions }\end{array}$ & $\begin{array}{l}\text { Dimensional } \\
\text { Contribution to } \\
\text { prediction of } \\
\text { outcome in the } \\
\text { full model }\end{array}$ & $\begin{array}{l}\text { Estimation of } \\
\text { predictive power } \\
\text { of categorical } \\
\text { (diagnoses) }\end{array}$ & $\begin{array}{l}\text { Diagnoses } \\
\text { Contribution } \\
\text { to prediction } \\
\text { of outcome in } \\
\text { the full model }\end{array}$ \\
\hline \multirow{6}{*}{$\begin{array}{l}\text { Dikeos } 2006 \\
\text { N=191 } \\
\text { Mixed stage sam- } \\
\text { ple }\end{array}$} & $\begin{array}{l}\text { OPCRIT outcome items: all } \\
\text { available information }\end{array}$ & \multicolumn{2}{|l|}{$\begin{array}{l}\text { PCA of OPCRIT life- } \\
\text { time psychopa- } \\
\text { thology }\end{array}$} & \multicolumn{2}{|l|}{$\begin{array}{l}\text { Clinical ascribed } \\
\text { Schizophrenia or } \\
\text { affective psychosis }\end{array}$} \\
\hline & \multirow{2}{*}{$\begin{array}{l}\text { Incomplete remission } \\
\text { Deterioration from premor- } \\
\text { bid level }\end{array}$} & Pseudo-R sq 0.270 & $\mathrm{P}<0.01$ & Pseudo-R sq 0.232 & NSS \\
\hline & & Pseudo-R sq 0.251 & NSS & Pseudo-R sq 0.265 & NSS \\
\hline & \multirow[t]{2}{*}{ No response to neuroleptics } & & & & \multirow[t]{2}{*}{ NSS } \\
\hline & & Pseudo-R sq 0.085 & NSS & Pseudo-R sq 0.060 & \\
\hline & Course & Pseudo-Rsq 0.358 & $\mathrm{P}<0.001$ & Pseudo-R sq 0.254 & NSS \\
\hline \multirow{5}{*}{$\begin{array}{l}\text { Rosenman } 2003 \\
\text { N=980 } \\
\text { Mixed stage sam- } \\
\text { ple }\end{array}$} & $\begin{array}{l}\text { Sum scores of non standard- } \\
\text { ised interview (last year) }\end{array}$ & \multicolumn{2}{|l|}{$\begin{array}{l}\text { PFA of SCAN cross } \\
\text { sectional psychopa- } \\
\text { thology in last month }\end{array}$} & \multicolumn{2}{|l|}{$\begin{array}{l}\text { OPCRIT generated } \\
\text { Diagnoses (DSM } \\
\text { IIIR) }\end{array}$} \\
\hline & Service demand & \multicolumn{2}{|l|}{$\mathrm{R}^{2}=0.085$} & \multicolumn{2}{|l|}{$\mathrm{R}^{2}=0.069$} \\
\hline & $\begin{array}{l}\text { Use of for support services } \\
\text { (sum score) }\end{array}$ & \multicolumn{2}{|l|}{$\mathrm{R}^{2}=0.001$} & \multicolumn{2}{|l|}{$\mathrm{R}^{2}=0.039$} \\
\hline & $\begin{array}{l}\text { Deteriorating course(sum } \\
\text { score) }\end{array}$ & \multicolumn{2}{|l|}{$\mathrm{R}^{2}=0.11$} & \multicolumn{2}{|l|}{$\mathrm{R}^{2}=0.139$} \\
\hline & $\begin{array}{l}\text { Social \& occupational func- } \\
\text { tional score }\end{array}$ & \multicolumn{2}{|l|}{$\mathrm{R}^{2}=0.237$} & \multicolumn{2}{|l|}{$\mathrm{R}^{2}=0.078$} \\
\hline \multirow{8}{*}{$\begin{array}{l}\text { Van Os } 1999 \\
\mathrm{~N}=706 \\
\text { Mixed stage sam- } \\
\text { ple }\end{array}$} & LCS (previous 2 years) WHO & \multirow{2}{*}{\multicolumn{2}{|c|}{$\begin{array}{l}\text { PCFA OPCRIT } \\
\text { Lifetime ratings }\end{array}$}} & \multirow{2}{*}{\multicolumn{2}{|c|}{$\begin{array}{l}\text { OPCRIT derived } \\
\text { RDC diagnosis }\end{array}$}} \\
\hline & DAS scale & & & & \\
\hline & Social disability & $\mathrm{R}^{2}=0.35$ & $\mathrm{p}<0.0005$ & $\mathrm{R}^{2}=0.26$ & NSS \\
\hline & Unemployed thought-out & Pseudo R-sq 0.13 & $\mathrm{P}<0.05$ & Pseudo R-sq 0.11 & NSS \\
\hline & Months independent living & $\mathrm{R}^{2}=0.11$ & $\mathrm{P}<0.005$ & $\mathrm{R}^{2}=0.08$ & NSS \\
\hline & $\begin{array}{l}\text { Incomplete remission no } \\
\text { recovery }\end{array}$ & Pseudo R-sq 0.17 & $\mathrm{P}<0.05$ & Pseudo R-sq 0.20 & NSS \\
\hline & Chronic course & Pseudo R-sq 0.12 & $\mathrm{P}<0.0005$ & Pseudo R-sq 0.06 & NSS \\
\hline & Days in hospital & $\mathrm{R}^{2}=0.05$ & NSS & $\mathrm{R}^{2}=0.07$ & NSS \\
\hline \multirow{9}{*}{$\begin{array}{l}\text { Van Os } 1996 \\
\mathrm{~N}=337 \\
\text { Recent onset } \\
\text { within } \\
5 \text { years of presen- } \\
\text { tation. }\end{array}$} & $\begin{array}{l}\text { LCS (previous } 2 \text { years) WHO } \\
\text { DAS scale }\end{array}$ & \multicolumn{2}{|l|}{$\begin{array}{l}\text { PCA using OPCRIT } \\
\text { Lifetime }(<5 \text { years } \\
\text { since presentation })\end{array}$} & $\begin{array}{l}\text { OPCRIT derived } \\
\text { DSM IIIR }\end{array}$ & \\
\hline & $\begin{array}{l}\text { Negative symptoms usually } \\
\text { present }\end{array}$ & PPV 0.773 & $\mathrm{P}<0.001$ & PPV 0.711 & NSS \\
\hline & Full recovery & PPV 0.688 & $\mathrm{P}<0.001$ & PPV 0.552 & NSS \\
\hline & Chronic course & PPV 0.712 & $\mathrm{P}<0.001$ & PPV 0.655 & NSS \\
\hline & Time employed & $\mathrm{R}^{2}=0.191$ & $\mathrm{P}<0.001$ & $\mathrm{R}^{2}=0.0 .09$ & NSS \\
\hline & Employed during follow up & PPV 0.639 & $\mathrm{P}<0.001$ & PPV 0.04 & $\mathrm{P}<0.001$ \\
\hline & Time as inpatient & $\mathrm{R}^{2}=0.08$ & $\mathrm{P}<0.001$ & $\mathrm{R}^{2}=0.04$ & NSS \\
\hline & Time living independently & $\mathrm{R}^{2}=0.118$ & $\mathrm{P}<0.001$ & $\mathrm{R}^{2}=0.029$ & NSS \\
\hline & DAS score & $\mathrm{R}^{2}=0.312$ & $\mathrm{P}<0.001$ & $\mathrm{R}^{2}=0.22$ & $\mathrm{P}<0.001$ \\
\hline
\end{tabular}

Definitions: PCA : principal component analysis, PFA principal factor analysis, PCFA principal component factor analysis, PPV positive predictive value taken from classification table of logistic regression. WHO DAS (disability assessment schedule), RDC (research diagnostic criteria) NSS not statistically significant. 


\section{OUTCOME}

The application of mixture modeling techniques for data reduction purposes, on outcome at 5 years, yields 3 ordered classes, which vary by level of outcome severity, from best to worst (most severe course and disability). There were clear monotonic change in means for longest time psychotic, time in residential support and time unemployed (or unable to carry out usual structured activities) and the conditional probabilities of class members endorsing all other outcomes showed a similar gradient. The only evidence of discordance was higher probabilities of endorsing severe ratings for the positive psychotic symptoms of delusional thought, paranoid ideation and Schneiderian first rank symptoms for the intermediate class while the worst outcome class had higher probabilities of endorsing moderate severity. These associations between symptom indicators and social disabilities are unlikely to be due to rater mediated information bias, as a different rater rated the symptom course measures than rated the social disabilities (i.e. independent ratings).

Latent class analysis makes no further assumptions about the underlying latent structure of outcome. Ordinal classes suggest a uni-dimensional outcome gradient, but are not sufficient evidence. Heterogeneity within classes (indicating configurationally different classes) may occur even in the presence of monotonicity across classes, due to sub-class factor structures, which can only be explored by hybrid latent modelling. Latent classes will however, adequately reduce the outcome data, allowing the testing of competing psychopathology-derived models (dimensions and diagnoses). The 3 class model was used in the ordinal regression analyses, however the 4 class, model is simply an elaborations of the model presented here, the poor outcome class remaining constant throughout the series of models fitted (not presented but solution can be provided on request). It is important to note the classes identified may be sample-specific and should be regarded as heuristic devices or constructs to aid our analyses and understanding, rather than true structures in nature. This reservation notwithstanding, the mixture model does suggest associations between different outcome domains at 5 years. Further research examining outcome using fine-grained assessment of outcomes, as they emerge would be informative.

\section{Methodological considerations}

The main limitation of the current study is the retrospective follow-up design. However, this study was carried using standardised schedules of outcome, designed to assess symptom course and disability retrospectively, in a region with integrated psychiatric services with low staff turnover, providing good longitudinal corroborative information. High quality, complete case notes were available, and $93 \%$ of those followed up had continued contact with at least one member of the community team at 5 years, so providing documentation throughout the period under study. At the time of assessment, patients would have different lengths of follow up depending on year of presentation. This could potentially result in recall bias, however, this is unlikely as contemporaneous clinical notes and service-administration details informed ratings of outcome. Further, no association was found between index diagnoses and year of presentation.

LTFU was primarily due to deaths in the first 5 years in patients with older age of onset psychosis - this may potentially bias towards worse outcome ${ }^{34,35}$. The high follow up rate at 5 years $(82 \%)$ makes it unlikely that explanatory models comparing dimensions and diagnoses will be compromised by differential drop-out. 


\section{CONCLUSION}

To the best of our knowledge, this is the first study to examine how well index diagnoses and dimensional representation of psychosis predict outcome as measured by standardised schedules of course and disability. The best predictive model employs current DSM IV diagnoses augmented with dimensional factor scores. Further work is required to examine i) differential variability of categories and dimensions predictive power across 'phase of illness' as perhaps dimensions are more useful in predicting and therefore guiding management after the initial presentation, ii) the best method to rate dimensions in clinical settings to capitalise in this small but potentially important added contribution to the explanatory power of diagnoses.

\section{Funding}

This study was supported by a grant from The Chief Scientist Office of the Scottish Government

\section{Acknowledgements}

Members of the D\&G 464 Study Group were:

- The Department of Clinical Research, Crichton Royal Hospital, Dumfries, Scotland, UK

- Robin McCreadie, Brain Hart, Gary Morrison, , Somashekara Shivashankar, Kartini Nor, Jamie Henderson, Valerie Sharkey, Catherine Colwell, Heather Stephens, Ciara Kelly, Mary Muirhead, Diane Jamieson, Kathy Walker \& Graham Gault

- Medical College of Georgia, USA

- Brain Kirkpatrick

- ISD Scotland, The Gyle, Edinburgh, EH12 9EB

- Denise Hastie, Lorraine Shark Diane Stockton

\section{REFERENCE LIST}

1. Hyman SE. Can neuroscience be integrated into the DSM-V?. Nature Reviews Neuroscience 8(9):725-32, 2007 September.

2. Andreasen NC. The validation of psychiatric diagnosis: new models and approaches. American Journal of Psychiatry 152(2):161-2, 1995 February.

3. $\quad$ Kendell R.E. Clinical validity. Psychological Medicine 1989;(1):45-55.

4. Kendler KS. The nosologic validity of paranoia (simple delusional disorder). A review. Archives of General Psychiatry 37(6):699-706, 1980 June.

5. Robins E, Guze SB. Establishment of diagnostic validity in psychiatric illness: its application to schizophrenia. American Journal of Psychiatry 126(7):983-7, 1970 January.

6. Allardyce J, Suppes T, van Os J. Dimensions and the psychosis phenotype. [Review] [72 refs]. International Journal of Methods in Psychiatric Research 16 Suppl 1 2007;S34-S40.

7. Stauss J.S., Carpenter W.T. Predicting the outcome of schizophrenia: 1 Characteristics of outcome. Archives of General Psychiatry. 27, 739-746. 1972.

Ref Type: Journal (Full)

8. Strauss JS, Carpenter WT, Jr. The prognosis of schizophrenia: rationale for a multidimensional concept. Schizophrenia Bulletin 4(1):56-67, 1978.

9. Carpenter WT, Bartko JJ, Strauss JS, Hawk AB. Signs and symptoms as predictors of outcome: a report from the International Pilot Study of Schizophrenia. American Journal of Psychiatry 135(8):940-4, 1978 August. 
10. Harding CM, Brooks GW, Ashikaga T, Strauss JS, Breier A. The Vermont longitudinal study of persons with severe mental illness, I: Methodology, study sample, and overall status 32 years later. American Journal of Psychiatry 144(6):718-26, 1987 June.

11. Allardyce J, Morrison G, Van Os J, Kelly J, Murray RM, McCreadie RG. Schizophrenia is not disappearing in southwest Scotland. Br J Psychiatry 2000;177:38-41.

12. Allardyce J, Boydell J, van Os J et al. Comparison of the incidence of schizophrenia in rural Dumfries and Galloway and urban Camberwell. British Journal of Psychiatry 179:335-9, 2001 October.

13. Kirkpatrick B, Tek C, Allardyce J, Morrison G, McCreadie RG. Summer birth and deficit schizophrenia in Dumfries and Galloway, southwestern Scotland. . American Journal of Psychiatry 159(8):1382-7, 2002 August.

14. Allardyce J, McCreadie RG, Morrison G, van Os J. Do symptom dimensions or categorical diagnoses best discriminate between known risk factors for psychosis? Social Psychiatry \& Psychiatric Epidemiology 42(6):429-37, 2007 June.

15. McGuffin P, Farmer A, Harvey I. A polydiagnostic application of operational criteria in studies of psychotic illness. Development and reliability of the OPCRIT system. Archives of General Psychiatry 48(8):764-70, 1991 August.

16. McGorry PD, Bell RC, Dudgeon PL, Jackson HJ. The dimensional structure of first episode psychosis: an exploratory factor analysis. Psychological Medicine 1998 July;28(4):935-47.

17. Cardno AG, Jones LA, Murphy KC et al. Dimensions of psychosis in affected sibling pairs. Schizophrenia Bulletin 1999;25(4):841-50.

18. Peralta V, Cuesta MJ. Dimensional structure of psychotic symptoms: an item-level analysis of SAPS and SANS symptoms in psychotic disorders. Schizophrenia Research 1999 July 27;38(1):13-26.

19. Harrison G, Hopper K, Craig T et al. Recovery from psychotic illness: a 15- and 25-year international follow-up study. British Journal of Psychiatry 178:506-17, 2001 June.

20. World Health Organisation. Life Chart Scedule. Geneva: WHO; 1992.

21. Levinson DF, Mowry BJ, Escamilla MA, Faraone SV. The Lifetime Dimensions of Psychosis Scale (LDPS): description and interrater reliability. Schizophrenia Bulletin 28(4):683-95, 2002.

22. Muthen LK, Muthen B.O. Mplus User's Guide. Los Angeles: Muthen \& Muthen; 2006.

23. Peralta V, Cuesta MJ. How many and which are the psychopathological dimensions in schizophrenia? Issues influencing their ascertainment. Schizophrenia Research 49(3):269-85, 2001 April 30.

24. Dikeos DG, Wickham H, McDonald C et al. Distribution of symptom dimensions across Kraepelinian divisions. British Journal of Psychiatry 189:346-53, 2006 October.

25. McIntosh AM, Forrester A, Lawrie SM et al. A factor model of the functional psychoses and the relationship of factors to clinical variables and brain morphology. Psychological Medicine 2001 January;31(1):159-71.

26. Murray V, McKee I, Miller PM et al. Dimensions and classes of psychosis in a population cohort: a four-class, fourdimension model of schizophrenia and affective psychoses. Psychological Medicine 35(4):499-510, 2005 April.

27. Peralta V, Cuesta MJ, Giraldo C, Cardenas A, Gonzalez F. Classifying psychotic disorders: issues regarding categorial vs. dimensional approaches and time frame to assess symptoms. European Archives of Psychiatry \& Clinical Neuroscience 2002 February;252(1):12-8.

28. Ratakonda S, Gorman JM, Yale SA, Amador XF. Characterization of psychotic conditions. Use of the domains of psychopathology model. Archives of General Psychiatry 1998 January;55(1):75-81.

29. Rosenman S, Korten A, Medway J, Evans M. Dimensional vs. categorical diagnosis in psychosis. Acta Psychiatrica Scandinavica 2003 May;107(5):378-84.

30. van Os J, Fahy TA, Jones P et al. Psychopathological syndromes in the functional psychoses: associations with course and outcome. Psychological Medicine 1996 January;26(1):161-76.

31. van Os J, Gilvarry C, Bale R et al. A comparison of the utility of dimensional and categorical representations of psychosis. UK700 Group. Psychological Medicine 1999 May;29(3):595-606.

32. Angst J. Today's perspective on Kraepelin's nosology of endogenous psychoses. European Archives of Psychiatry \& Clinical Neuroscience 243(3-4):164-70, 1993. 
33. Susser E, Finnerty M, Mojtabai R et al. Reliability of the life chart schedule for assessment of the long-term course of schizophrenia. Schizophrenia Research 42(1):67-77, 2000 March 16.

34. Watt DC, Szulecka TK. The effect of sex, marriage and age at first admission on the hospitalization of schizophrenics during 2 years following discharge. Psychological Medicine 9(3):529-39, 1979 August.

35. Soni SD, Gaskell K, Reed P. Factors affecting rehospitalisation rates of chronic schizophrenic patients living in the community.[erratum appears in Schizophr Res 1995 Jan;14(2):185]. Schizophrenia Research 12(2):169-77, 1994 May. 
CHAPTER 11

Epilogue \& Future directions for Epidemiological Research 


\section{EPILOGUE \& FUTURE RESEARCH DIRECTIONS}

Classical epidemiological studies of environmental risk factors: $<{ }^{*}$ I $^{*}$ The findings from the studies described and reviewed in this thesis show a number of environmental factors which are consistently $\left.<^{*} I^{*}\right\rangle$ statistically associated $\left\langle{ }^{*} I^{*}\right\rangle$ with an increased risk for the onset of schizophrenia and/or other non affective psychotic disorders, but with only small to moderate effect sizes.

TABLE 1: summary of the most often studied environmental risk factors

\begin{tabular}{|c|c|c|c|c|}
\hline Risk Factor & $\begin{array}{l}\text { Effect size } \\
\text { (pooled) }\end{array}$ & $\begin{array}{l}\text { Dose } \\
\text { Response }\end{array}$ & Temporal Association & $\begin{array}{l}\text { Replicated } \\
\text { \& consistent finding }\end{array}$ \\
\hline \multicolumn{5}{|c|}{ Risk Factors Operating during early development } \\
\hline Paternal Age & $\mathrm{OR}=2$ & $\checkmark$ & $\checkmark$ & $\checkmark$ \\
\hline $\begin{array}{l}\text { Season of birth } \\
\text { In Northern hemi- } \\
\text { sphere }\end{array}$ & $\mathrm{OR}=1.07$ & $\sim$ & $\checkmark$ & $\begin{array}{l}\text { Replicated in Northern } \\
\text { Hemisphere } \\
\checkmark\end{array}$ \\
\hline $\begin{array}{l}\text { Pregnancy \& birth } \\
\text { complications }\end{array}$ & $\mathrm{OR}=2$ & $\sim$ & $\checkmark$ & $\checkmark$ \\
\hline \multicolumn{5}{|c|}{ Risk Factors operating during childhood and early adolescence } \\
\hline $\begin{array}{l}\text { Urban birth \& up- } \\
\text { bringing }\end{array}$ & $\mathrm{OR}=2$ & $\checkmark$ & $\checkmark$ & $\checkmark$ \\
\hline Migration & $\begin{array}{l}\mathrm{OR}=2 \\
\left(1^{\text {st }} \text { gen }\right)\end{array}$ & NA & $\sim$ & $\checkmark$ \\
\hline Cannabis use & $\mathrm{OR}=1.4$ & $\checkmark$ & $\sim$ & $\checkmark$ (for psychotic experiences) \\
\hline
\end{tabular}

Furthermore, other environmental risk factors have been proposed and require further study to establish their association (prenatal infections, rhesus compatibility, prenatal maternal stress, stressful life events, early childhood neglect, physical brain trauma and societal organizational factors, taken together these studies suggest a possible environmental influence on the onset of schizophrenia/ psychosis. Gene environment correlation: Could the association of environmental risk factor exposure and schizophrenia/psychosis be confounded by genotype? However, While contemporary epidemiological studies of environmental risk factors have substantially improved designs which are likely to reduce bias and confounding from other known environmental risk factors, what cannot be adjusted for in studies designed to examine only environmental exposure is possible confounding by genotype. That is, the relationship seen for the environmental factor is due directly to the effect of the genotype (non-causal gene environment correlation). 


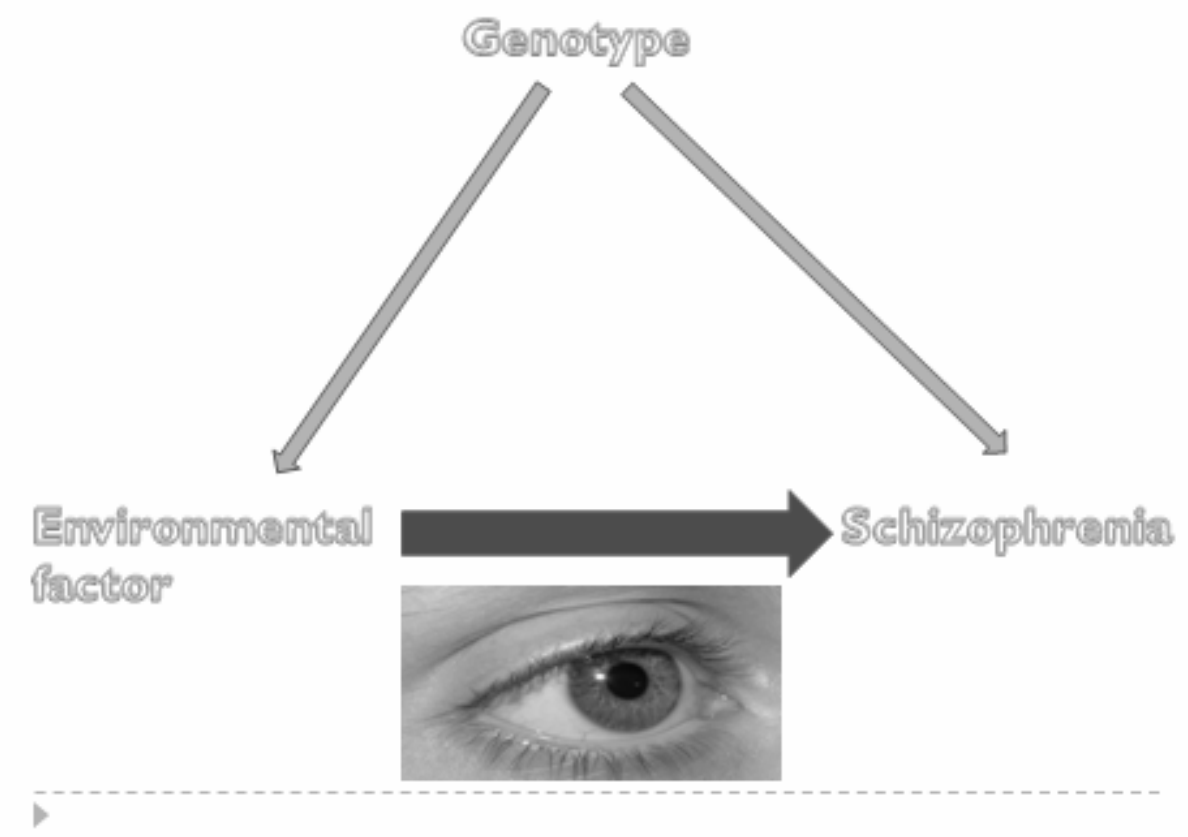

Figure 1: Gene environment correlation (non causal gene environmental correlation)

This is especially a problem in studies of environmental risk factors for diseases which have a, significant genetic predisposition. In schizophrenia there is convincing evidence from family, twin and adoption studies demonstrating a high genetic predisposition with heritability estimates of around $80 \%$ (derived from twin studies). On the other hand however, such heritability estimates do not model joint gene -environment influences, therefore it is quite possible that joint gene environment effects explain a substantial proportion of such estimates. Furthermore, to date (but perhaps this will have changed by the time of the PhD Defense!) there have been no genes identified which have consistently shown a moderate /strong main effect association with schizophrenia/psychosis, at the population level. (Craddock, O’Donovan et al. 2006).

\section{POSITED THEORY FOR THE ALLELIC ARCHITECTURE OF SCHIZOPHRENIA}

\section{Polygenetic Transmission and schizophrenia}

The common disease-common variant (CD-CV) hypothesis posits that the genetic susceptibility to disease is largely due to alleles which have a moderate frequency in the population. This is a pattern of genetic transmission which is found repeatedly in nature with multiple genes influencing complex traits such as psychosis (through additive or multiplicative effects of small magnitude) The model suggests that genetic variation influencing disease phenotypes will follow a distribution in the population with many rare variants and some common variants. Even if larger effect sizes were found for rare variants, because common variants are more prevalent they will explain the large majority of cases within a population (i.e. the common variants will have a higher population attribution risk, assuming causality).If the CD-CV model approximates to the allelic architecture of schizophrenia, genome wide association studies (GWAS) should be a powerful tool for detecting such loci of small effect, while if there is substantial allelic heterogeneity due to rare variants or epigenetic phenomena (low allelic identity) association mapping would be very much less effective, because each mutation would likely arises on an independent haplo- 
type background (therefore tending to cancel out each other's signals) (Lander 1996; Chakravarti 1999).

SNP (single nucleotide polymorphisms.) To date, the most frequently studied CV employ SNP markers. Studies of variation in the human genome indicate that we all share approximately $99.9 \%$ of our DNA sequence, while this seems like a very small difference because the human genome is around 3.2 billion base pairs in magnitude; this translates to you having 3 million base pairs which are different to mine! A site in the genome where individual members of a species differ in a single base pair is called a SNP ("snip"). SNPs arise from mutations which are then inherited through allelic variants, which generally do not produce phenotypic variation. This results in each SNP being associated with other specific SNPs (as well as other types of genetic variants and alleles) present on the chromosome where the mutation occurred, this set of linked alleles is termed a Haplotype. SNPs within a haplotype block are physically linked and therefore tend to be inherited together. New haplotypes do arise from mutations but less commonly arise from crossing over (and the rate of crossing over is proportional to the distance between the genes), therefore SNPs and other variants which are physically close together on the chromosome will be strongly associated as haplotypes. The non random association between genetic variants within a haplotype is termed linkage disequilibrium. Because of their variability and widespread occurrence throughout the genome SNPs which are common polymorphisms (definition : occurring at $>=1 \%$ of the population) are valuable markers in linkage and association studies. When a SNP is physically close to a disease causing locus it will tend to be inherited along with the disease causing allele. People with the disease will tend to have different SNPs from those of non diseased people and this is the strategy used in gene fine mapping (Pierce 2008).Genome wide Association studies using SNP have been successful in detecting strong associations in common diseases such as coronary artery disease, hypertension, rheumatoid arthritis, Crohn's disease and Type 2 Diabetes mellitus.

CNVs Copy Number variation: Each diploid person normally possesses 2 copies of every gene; one inherited from their mother and the other form their father. However, studies of the human genome have revealed differences among people in the number of copies of large DNA sequences called copy-number variations (greater than 1000bp). CNVs include deletions; a person has a single copy or sequence, or duplications, when they have more than 2 copies of a particular gene. Data from the HapMap project indicates a relatively large number of copy number variants within the human genome. Many of these CNVs encompass large regions of DNA sequencing, often several thousand base pairs in length. This suggests that people differ not only at millions of different individual SNP regions, but also in the number of copies of many larger segments of the genome. Most CNVs contain multiple genes and potentially affect the phenotype by altering gene dosage and by changing the position of the sequences, which affect the regulation of other genes close to the altered region. Recent studies suggest that rare CNVs may be implicated in some cases of schizophrenia (Kirov, Gumus et al. 2008)suggesting that the CD-CV hypothesis may not account for all the genetic effects in schizophrenia but as posited earlier there are also rare variants with larger effects implicated.

Microsatellite variation: Microsatellites are short DNA sequences that exist in multiple copies repeated in tandem. People vary in the number of copies of repeat sequences that they possess. Work primarily stemming from DNA fingerprinting research in forensic pathology has demonstrated the variation in banding patterns in microsatellite sequences are inherited, with all the bands found in offspring also being present in the parents. This form of DNA sequence variation has been used to demonstrate evidence of gene environment correlation and evolutionary pressure in sport hunting trophy rams and is likely to be extended into the study of human populations (Pierce 2008). 
With ever advancing technologies for genotyping it is likely that the next decade will see the proliferation of GWAS studies using SNP, CNV, microsatellites, epigenetic and gene expression markers of genetic variation and perhaps just perhaps masses and masses of power will be enough to detect the genes involved in the development of schizophrenia, however it still does not solve the catch 22 situation: are our known environmental risk factors confounded by genotype or conversely are our genetic studies ignoring higher order interactions (gene-gene interactions (GxG) \& Gene-environment interactions (GxE)), which could be helpful in understanding the causes of schizophrenia?

\section{Genetic epidemiology: Examining the joint action of genes \& environmental factors in the cause of schizophrenia.}

Genetic epidemiology is the study of the joint actions of genes and environmental factors in causing disease in human populations and their patterns of inheritance in families. Family studies are the basic approach used by genetic epidemiologists but the basic designs of classical risk factor epidemiology (case control studies and cohort studies) are also useful for some purposes. While the, statistical and laboratory techniques involved in genetic and aetiological research are ever evolving, the basic epidemiological principles remain useful and necessary (Thomas 2004).

\section{Complex genetic interactions}

Traditional genetic epidemiology has focused on the identification of genetic markers that individually increase/decrease the occurrence of a disease (main effects). Yet for the majority of trait expression also depends at some level on the interaction with genes and/or environmental factors either where the interactions are genetically programmed or purely random. Even the phenotypic manifestations of conditions which have monogenetic inheritance may be the result of GxE e.g. phenylketanuria Schizophrenia is considered by most researchers in the field (though we do not know for sure) to be complex multifactorial disorder-therefore analytical strategies which are able to determine the cause of observed discordance between genetic susceptibility and phenotypic expression will be required. That is, our genetic models of disease need to take into consideration interactions with other genes and environmental factors and consider designs to deal with genetic heterogeneity. In order to do this we require methods that simultaneously consider the effects of multiple genes and environmental factors.

\section{Gene-Gene \& Gene-Environment Interactions:}

Definition of interaction The term interaction is used loosely in the current genetic and epidemiological literature. In general one can consider GxE equivalently as either increased (or decreased) vulnerability to environmental exposures in carriers of a certain genotype relative to non-carriers, or increased (or decreased) genetic expression in exposed subjects compared to unexposed subjects. Effect modification (also termed interaction) occurs when the measure of effect varies for one variable across levels of another variable. Clearly from this definition the precise meaning of the interaction depends on the scale on which the effect is measured (Thomas 2004).

Therefore statistical interaction means a departure of the observed risk for some model of main effects for 2 or more risk factors. However statistical interaction does not necessarily imply biological interaction and vice- versa. Nevertheless such interactions are often interpreted as having biological significance about underlying mechanisms (Ottman 1990; Yang and Khoury 1997). 


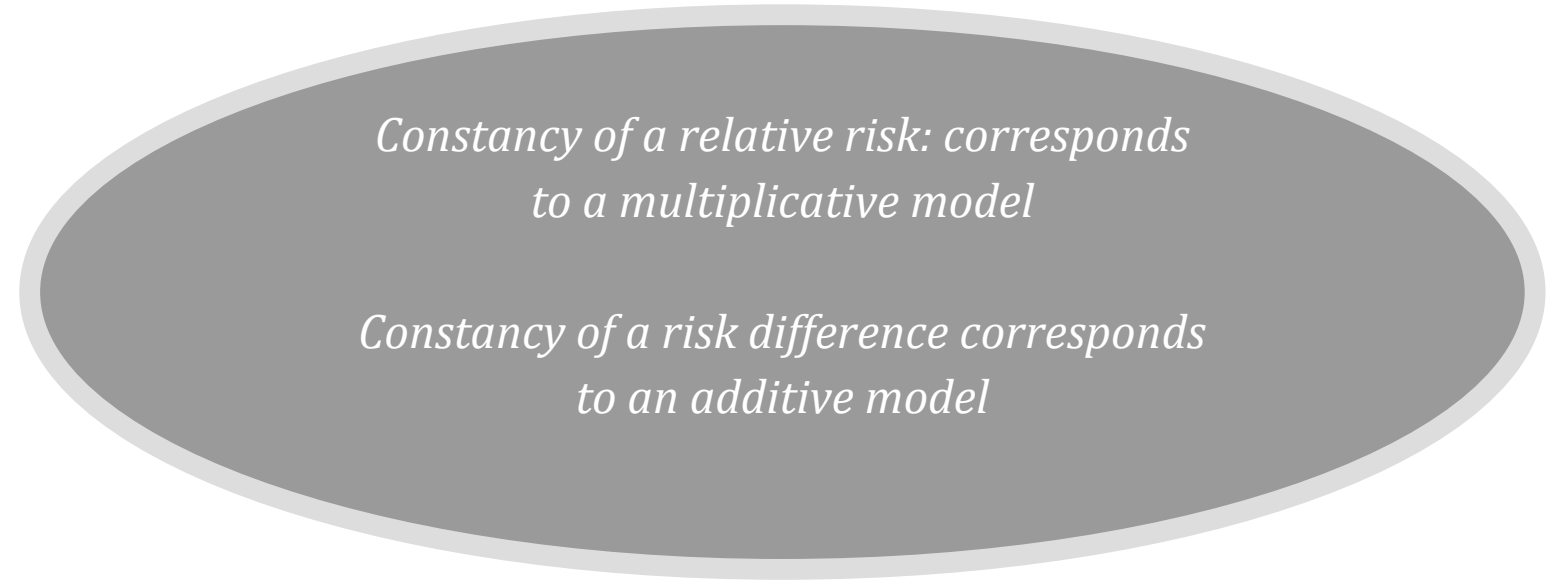

\section{Gene-Gene Interaction}

The polygenic model discussed above posits that multiple interacting genes are likely to influence the phenotype. However this polygenic inheritance is difficult to demonstrate in humans and complicates genetic mapping because no single locus is solely responsible for producing the trait. This means that in future research we will have to use a combination of analytical approaches to understand the complexity of the susceptibility to schizophrenia, including 1) to examine currently posited candidate genes which have been found to have main effects on the risk of developing schizophrenia for potential interactions using 2-locus linkage models or casecontrol/family association tests. However, studies which aim to detect novel complex interactions or replicate previously reported ones must have much larger sample sizes than studies designed to detect only main effects. (Smith and Day 1984). 2) Whole genome screen approach to identify addition loci conditional on a genotype previously shown to be of genetic risk in schizophrenia, these methods are powerful at finding markers with small effect that combine to have an important effect on the phenotype, however they do not explicitly look for GxG or GxE interaction. 3) exhaustive searching approaches which consider many genetic factors at once and use latent variable modeling techniques to identify patterns such as dimensional reduction/combinational portioning and class identification /regression trees and convergent pathway analysis that best predict the disease phenotype from genotypic information, these methods seem to work best with small number of markers $(<10,000)$ and will have to be developed to include explorative methods with larger marker coverage 4) ELA (ensemble learning approach) (Zhaogong Zhang 2008) and their extensions are being developed for this purpose.

\section{GENE -ENVIRONMENT INTERACTION (EXPLORATION OF MULTIFACTORIAL MODELS)}

\section{"Genetic s loads the gun and the environment pulls the trigger"}

In this thesis I have discussed the indirect evidence from epidemiological studies suggesting the possibility of GxE interactions in the onset of psychosis. Once a major gene or environmental factor has been identified GxE can be explored. The examination of the candidate genes in the study of disease -exposure associations or GxE interactions can reveal effects of an environmental factor on the risk of schizophrenia that would be concealed if genetic susceptibility was ignored. Furthermore, considering an established environmental risk factor may facilitate the 
identification of novel genetic factors (Haines 2006). To date most models of gene-environment interaction are simple, and consider the effect of only one gene and one environmental risk factor. In reality the interactions are likely to be much more complex, involving multiple genes, environmental factors and will have to consider genetic heterogeneity and heterogeneity of exposure. The plausible GxE models described to date however provide us with a framework for discussing potential study designs and approaches of more complex interactions which will be developed over the next decade.

\section{Generic heterogeneity}

Is present when a number of different mutations $\rightarrow$ identical phenotypes. Or phenotypes that are similar enough to have been traditionally lumped together under one diagnosis. There are 2 types of genetic heterogeneity Allelic Heterogeneity is common phenomena in nature occurs when different mutations at a single locus cause the same phenotype. Locus heterogeneity occurs when mutations at different loci cause the same phenotype or a group of phenotypes which appear similar and have traditionally been classified as a single disorder/ or spectrum. Much can be learned from this when a distinctive phenotype is produced by alleles at more than one gene locus, it suggests that the gene products interact in some way or that they are necessary components of a critical developmental or metabolic pathway -causal mechanisms.

\section{Potential Methodologies: Association studies including complex putative component causes}

As discussed above, the majority of GxE interaction studies have used methodologies in which there are only a few risk factors examined at a time, the most commonly used approaches are case control designs allows the examination of the joint effects of genetic and environmental factors on disease susceptibility while theoretically more complex models incorporating both GxE and GxG interactions and even higher order (GxGxE) with multiple genes and environmental exposures are possible using this framework, pragmatically data collection (such as genotyping for the controls that are likely to yield a low number of carriers may be costly), the multiplicity of hypothesis, and confounding by recent admixture, selection and drift between unlinked loci make this methodology relatively in-efficient except in the situation where linkage studies have identified a chromosomal region likely to be implicated i.e. the candidate gene approach. . Caseonly design can also be used to examine GxE interaction is the case only studies. This approach can improve the power of the study to detect interactions and is valid for rare diseases. However importantly it is only valid when there are strong a priori reasons to believe that the genotype and the environmental exposure are independent of each other in the study population. (Piegorsch, Weinberg et al. 1994; Yang and Khoury 1997). Violation of this assumption has been shown to produce grossly inflated type 1 errors. A preliminary assessment of the independence of the 2 factors being studied can be performed by examining the co variation of the genotype and environmental exposure in a sample of people who do not have the disorder. However even when a test of independence is carried out in control subjects the bias may persist (Albert, Ratnasinghe et al. 2001).The case only approach is therefore only useful for assessing departures from the multiplicative interaction when there is a strong empirical evidence for the gene environment independence.

Family studies: case -example of parent trios: The case parent trio design has been used to test candidate gene associations but is also used for testing GxG and GxE interactions, under a weaker gene environment independence assumption than that required by the case only design. This model uses the genotypes on all 3 members of the trio but ONLY the environmental exposure from the case (I am not really sure what a cases control comparison between the cases and 
the parents exposure would mean anyway as you would not be comparing like for like by age, gender and other demographics). Basically the design stratifies the genetic relative risk estimates from the case-parent trio by environmental exposure status of the case, if there is no GxE interaction the 2 genetic relative risks would be the same, however if an interaction is present their ratio will estimate the interactive relative risk (i.e. a comparison the genetic relative risk estimated from environmental exposed and unexposed cases). Currently we can use stratified analysis to control for known within family variables which may influence the risk and multilevel analytical models are currently being developed to deal with these factors (Thomas 2004; Haines 2006)

\section{Genome wide scans and association and interaction}

The studies described above are all hampered by lack of reproducibility due to 1)too small samples with inadequate statistical power to uncover complex interactions and small effects,2) the biological and phenotypic complexity of schizophrenia, 3) population -specific linkage disequilibrium, 4) effect size bias, and 5) differing population substructures (Davey Smith, Ebrahim et al. 2005; Crow 2007) furthermore methodological heterogeneity, such as variation in diagnostic criteria used to define the disease phenotype, different methods for measuring environmental exposure and variation in the analyses and the choice of markers chosen will only aggravate this. These methodological concerns in parallel with the development of high volume genotyping technologies (which make explorative studies of genetic variation across the entire human genome feasible) point towards GWAS (Genome wide association studies) studies as being potentially useful way of identifying genetic associations with disease status. The design of such studies will be fundamentally the same as traditional genetic epidemiology, however the ambitiousness of such projects present a number of scientific and logistical challenges (and probably also political-this will require cross department cross national collaboration-good luck!) The power calculations suggest numbers in the thousands will be required to carry out such studies (Collins 2004) however coordinated infra structures for genetic association studies are being constructed (ISC; SGENE). To be clinically relevant these genetic association studies will need to be placed in an epidemiological, psychiatric and public health context. However, as yet no statistical models or software which would allow us to concurrently look across the genome and test for environmental sensitivity (GxE) has been developed and this will require collaboration with genetic statisticians to develop appropriate analytical models to deal with such problems as 1) multiple comparisons which may be further accentuated if many GxG or GxE interactions are also considered and it may be that progress can only be made in the characterization of complex diseases if we consider such interactions even when only limited a priori knowledge about the range of exposures is minimal. A variety of data mining techniques have been proposed for this purpose, such as artificial neuronal networks, path \& cluster analysis methods (Ritchie, Hahn et al. 2001; Moore, Parker et al. 2002; Moore and Williams 2002; Jansen 2003) 2) population stratification 3) heterogeneity of effect due to unrecognized modifying factors either genetic or environmental. It will be important in the reporting of such studies to consider the potential sources of bias and confounding and report associations that make biological sense and alleles that affect the gene product in a physiologically meaningful way. An anonymous editorial in Nature Genetics (1999) suggested that the associations should be observed both in families and population based studies and the effect size should be high (for rare variants) and the population attributable risk high for common variants before being reported and published. 
As well as considering the joint gene and environmental risk factors effect for risk of schizophrenia, as epidemiologists we will have to better characterize our environmental exposures, this is expensive and time consuming and furthermore will require the development of better more fine grained assessment tools with increased precision. To date, we have focused primarily on exposure at the level of the individual however increasingly studies examining complex disease epidemiology has shifted away from previous individual risk factor emphasis, to a model which investigates causal pathways across societal, individual and molecular levels (Susser and Susser 1996; Susser and Susser 1996).Chapters in this thesis have examined area level variation in urbanicty/migrant status and social fragmentation. Further studies are required to disentangle wither these are contextual effects (mediated by perhaps social defeat or other social network/integration factor, (Selten and Cantor-Graae 2007) or are simply area level proxy indicators for as yet unidentified individual environmental exposures/mediating factors which are more prevalent in such areas (i.e. aggregate effect). This will require the development of methodologies to deal with the significant heterogeneity of effect, perhaps by stratified or sub group sampling of certain environmental exposures. While rationally epidemiological studies have tended to examine exposure to outcome without integrating intermediate factors on the causal pathway or causal mechanisms the methods described above will facilitate the integration of paradigms which test the evolving pharmacological hypotheses for schizophrenia.

\section{What phenotype should be studied!}

The importance of case definition cannot be over emphasized and is an essential requirement for genetic epidemiological studies. This thesis discusses the relatively arbitrary development of the traditional classification systems for psychosis which have some clinical usefulness and are based on symptom cluster and relevance to functioning. However, understanding the complex genetic causes of schizophrenia will likely require phenotypic definitions which are based/ or are closer to pathogen tic mechanisms. Further we have to consider the possibility that not all clinical features are equally as important in defining phenotypes. The studies in this thesis examine the usefulness of symptom based dimensional measures generated using latent variable modeling techniques of exploratory factor analysis. Future studies are likely to examine both dimensional (factor models) and categorical (latent class) methods to better improve our classification systems, but such studies will have to more carefully consider the indicators being used to tap into the latent level structures. Just because a symptom is severe/ or clinically relevant does not mean that it is a good indicator for discriminating underlying etiologies. Minor clinical features which do not have important medical or functional implications may become important when defining our psychotic phenotypes. Many medical syndromes are defined by their patterns of minor features rather than major clinical features. Furthermore if in family studies we find a number of minor clinical features segregating with major psychosis it may suggest pleiotrophy, and the minor clinically trivial features may be of help in phenotypic definition. This thesis demonstrates that current classification systems explain only relatively small amounts of the risk factor and prognosis ( 5 years follow up) variance, the best model using information from both diagnoses and dimensional models explains only $25 \%$ of the variation in outcome/risk factor differences. In order to develop definitions which are more aetiologically relevant intermediate phenotypes may be useful, e.g. neurocognitive impairment (Gottesman and Gould 2003; Greenwood, Braff et al. 2007).and structural brain abnormalities (Boos, Aleman et al. 2007) have been found to aggregate in families and in some case has been found to be related to specific genetic 
variants and it is likely that these profiles could be used fine tune out definition of caseness in aetiological studies may even be useful outcome variables.

\section{Psychosis evolution: a dynamic process}

That at-risk mental state, characterized by brief low grade psychotic experiences (in late childhood early adolescence) predict later onset of psychotic disorder is now a replicated finding. Over the next decade epidemiological research will model the preclinical and clinical characteristics which influence the transition to disorder and need for health care, this will be facilitated with the development of powerful latent variable growth mixture models which use normal random effects to represent the departure of an individual's latent growth parameters from the population mean growth parameters, so that class membership of possible subgroups of psychosis while not being measured directly, can be inferred by their developmental courses which will have covariates relationships which may be useful in classification and prediction models. This may result in the identifying of multiple developmental pathways and the examination of environmental and genetic co-variate effects on the different population subgroups.

\section{REFERENCE LIST}

Albert, P. S., D. Ratnasinghe, et al. (2001). "Limitations of the case-only design for identifying gene-environment interactions." American Journal of Epidemiology 154(8): 687-93.

Boos, H. B. M., A. Aleman, et al. (2007). "Brain volumes in relatives of patients with schizophrenia: a meta-analysis." Archives of General Psychiatry 64(3): 297-304.

Chakravarti, A. (1999). "Population genetics--making sense out of sequence.” Nature Genetics 21(1 Suppl): 56-60.

Collins, F. S. (2004). "The case for a US prospective cohort study of genes and environment." Nature 429(6990): 475-7.

Craddock, N., M. C. O’Donovan, et al. (2006). “Genes for schizophrenia and bipolar disorder? Implications for psychiatric nosology." Schizophrenia Bulletin 32(1): 9-16.

Crow, T. J. (2007). "How and why genetic linkage has not solved the problem of psychosis: review and hypothesis." American Journal of Psychiatry 164(1): 13-21.

Davey Smith, G., S. Ebrahim, et al. (2005). "Genetic epidemiology and public health: hope, hype, and future prospects.” Lancet 366(9495): 1484-98.

Gottesman, I. I. and T. D. Gould (2003). "The endophenotype concept in psychiatry: etymology and strategic intentions." American Journal of Psychiatry 160(4): 636-45.

Greenwood, T. A., D. L. Braff, et al. (2007). "Initial heritability analyses of endophenotypic measures for schizophrenia: the consortium on the genetics of schizophrenia." Archives of General Psychiatry 64(11): 1242-50.

Haines, J. L. P.-V., M.A. (2006). Genetic Analysis of Complex Diseases. New Jersey, John wiley \& Sons.

ISC. "http://pngu.mgh.harvard.edu/isc/."

Jansen, R. C. (2003). "Studying complex biological systems using multifactorial perturbation." Nature Reviews Genetics 4(2): 145-51.

Kirov, G., D. Gumus, et al. (2008). "Comparative genome hybridization suggests a role for NRXN1 and APBA2 in schizophrenia." Human Molecular Genetics 17(3): 458-65.

Lander, E. S. (1996). “The new genomics: global views of biology.[see comment].” Science 274(5287): 536-9.

Moore, J. H., J. S. Parker, et al. (2002). "Symbolic discriminant analysis of microarray data in autoimmune disease." Genetic Epidemiology 23(1): 57-69.

Moore, J. H. and S. M. Williams (2002). "New strategies for identifying gene-gene interactions in hypertension." Annals of Medicine 34(2): 88-95. 
Ottman, R. (1990). “An epidemiologic approach to gene-environment interaction.” Genetic Epidemiology 7(3): 177-85.

Piegorsch, W. W., C. R. Weinberg, et al. (1994). "Non-hierarchical logistic models and case-only designs for assessing susceptibility in population-based case-control studies." Statistics in Medicine 13(2): 153-62.

Pierce (2008). Genetics a conceptual approach. new York, W.H. Freeman and Company.

Ritchie, M. D., L. W. Hahn, et al. (2001). "Multifactor-dimensionality reduction reveals high-order interactions among estrogen-metabolism genes in sporadic breast cancer." American Journal of Human Genetics 69(1): 138-47.

Selten, J.-P. and E. Cantor-Graae (2007). "Hypothesis: social defeat is a risk factor for schizophrenia?" British Journal of Psychiatry - Supplementum 51: s9-12.

SGENE. “http://www.sgene.eu/."

Smith, P. G. and N. E. Day (1984). "The design of case-control studies: the influence of confounding and interaction effects." International Journal of Epidemiology 13(3): 356-65.

Susser, M. and E. Susser (1996). "Choosing a future for epidemiology: I. Eras and paradigms.[see comment][erratum appears in Am J Public Health 1996 Aug;86(8 Pt 1):1093].” American Journal of Public Health 86(5): 668-73.

Susser, M. and E. Susser (1996). "Choosing a future for epidemiology: II. From black box to Chinese boxes and ecoepidemiology.[see comment][erratum appears in Am J Public Health 1996 Aug;86(8 Pt 1):1093]." American Journal of Public Health 86(5): 674-7.

Thomas, D. C. (2004). Statistical methods in Genetic Epidemiology. New York, Oxford University Press.

Yang, Q. and M. J. Khoury (1997). "Evolving methods in genetic epidemiology. III. Gene-environment interaction in epidemiologic research." Epidemiologic Reviews 19(1): 33-43.

Zhaogong Zhang, S. Z., Man-Yu Wong, Nicholas J. Wareham, Qiuying Sha (2008). "An ensemble learning approach jointly modeling main and interaction effects in genetic association studies." Genetic Epidemiology 32: 285 - 300. 
Samenvatting (Summary in Dutch) 
Schizofrenie is een ernstige en vaak verzwakkende aandoening gekarakteriseerd door symptomen zoals hallucinaties, waanideeën, desorganisatie, verlies van doelgericht gedrag en afname in sociaal functioneren. Bij sommigen verloopt de ziekte relatief mild, waarbij de persoon een of enkele episodes doormaakt met weinig tot geen blijvende beperkingen. Echter, bij ongeveer $47 \%$ van de patiënten is schizofrenie een levenslange aandoening, met blijvende symptomen en terugkerende episodes geassocieerd met een significante sociale beperking. De oorzaak van schizofrenie blijft echter onvindbaar. Hoewel het duidelijk is dat er een sterke familiaire kwetsbaarheid bestaat waarbij eerstegraads familieleden van patiënten een hoger risico op morbiditeit voor schizofrenie hebben vergeleken met familieleden van controlepersonen. Schattingen variëren van $2-9 \%$ vergeleken met een risico voor familieleden van controles van ongeveer $0,5 \%$. Tweelingstudies ondersteunen deze aanname ook met concordantie cijfers van $50 \%$ in monozygotische tweelingen en $4,1 \%$ in dizygotische paren, echter de genetische bijdrage blijft nog steeds onduidelijk en omgevingsrisicofactoren zoals het opgroeien in een stad, de leeftijd van de vader, cannabis gebruik hoofdzakelijk tijdens adolescentie en obstetrische complicaties worden ook verondersteld een rol te spelen.

Variatie in ziekte en tijd Epidemiologische methoden vergelijken systematisch en zetten een verzameling ziekte patronen in populaties over TIJD, PLAATS en in verschillende PERSONEN.

Variatie in ziekte over tijd. Dat ziektes toenemen en afnemen in bevolkingsfrequentie als gevolg van medische/bevolkingsgezondheid praktijken of door natuurlijke oorzaken is een van de axiomen van de klassieke epidemiologie. Variatie in ziekte in de bevolking vindt plaats over tijd doordat eigenschappen van mensen of hun omgeving veranderen. Zelfs als de sociale en fysische omgeving constant zouden blijven zouden patronen in het voorkomen van ziekten over tijd voorkomen (natuurlijk veel trager, eerder evolutionaire tijdsschalen) omdat genetische veranderingen onvermijdelijk zijn en deze het ontstaan en de persistentie van een ziekte beïnvloeden. Genetische veranderingen ontstaan door een aantal verschillende processen waaronder genetische drift, genetische mutatie en epigenetische transmutaties, die random variatie in de genfrequentie veroorzaken van generatie op generatie. In kleine populaties kan genetische drift tot belangrijke genetisch aangestuurde ziekte verschillen leiden, echter in grotere populaties blijft de genetische DNA (deoxyribonucleic acid) sequentie relatief stabiel over tijd. De klassieke epidemiologische methodologie veronderstelt dat veranderingen in het voorkomen van ziekten in grote bevolkingen die plaatsvinden binnen korte tijd (tientallen jaren) geheel toe te schrijven zijn aan omgevingsfactoren. Hoofdstuk 2 van dit proefschrift onderzoekt de trend van incidentie van schizofrenie over tijd in Dumfries \& Galloway in de periode 1979-1989. In deze regio was er geen gradiënt te zien.

Variatie van ziekte in plaats Gelijke inzichten in de etiologie kunnen bepaald worden door systematisch de variatie van ziekte cijfers te vergelijken tussen populaties met verschillende demografische en omgevingskenmerken. In Hoofdstuk 34 \& 5 wordt deze spatiële variatie onderzocht en de resultaten suggereren dat in stedelijke gebieden en gebieden met lage sociale cohesie eerste psychotische episoden en schizofrenie vaker voorkomen.

Variatie in persoon (klinische epidemiologie: subgroep analysen) benut de inherente heterogeniteit die gezien wordt in populaties. In Hoofdstuk 6 van dit proefschrift zal ik de bestaande literatuur systematisch bestuderen en vinden dat, hoewel familiale kwetsbaarheid de grootste effecten heeft, er ook sterke bewijzen zijn dat paternale leeftijd een matig sterke invloed heeft, wat mogelijk wijst op het bestaan van epigenetische mechanismen dat verder onderzoek vereist. Bij elke epidemiologische studie staat het thema " patiënt zijn" centraal. Hoe we een ziekteuitkomst definiëren bepaalt de geschatte cijfers in beschrijvende epidemiologische studies en de berekende effect groottes in analytische studies naar risicofactoren. Vanaf 1960 hebben psychiaters veel energie besteedt aan het verbeteren van de betrouwbaarheid van psychiatrische diagnosen in algemeen, en speciaal van schizofrenie. Dit heeft geresulteerd in een aantal operatio- 
nele definities van schizofrenie met acceptabele betrouwbaarheid, maar allen variëren en het is twijfelachtig welke aangenomen moet worden. Het hoofdprobleem is dat we niet weten of het concept schizofrenie enige validiteit heeft, en als dat al zo is, welke operationele definitie de validiteit het best reflecteert. Dat schizofrenie op zijn best een voorlopige diagnostische categorie is heeft serieuze gevolgen voor het opzetten van epidemiologische studies en de interpretatie van resultaten. In Hoofdstuk 789 \& 10 van dit proefschrift onderzoek ik zowel theoretisch als empirisch de bruikbaarheid van categorische diagnosen in schizofrenie vergeleken met dimensionale gekwantificeerde symptoomprofielen. Deze studies suggereren dat er weinig te winnen is met het gebruiken van dimensionale maten alleen, hoewel ze licht kunnen bijdragen aan de voorspellende waarschijnlijkheid in uitkomst studies als ze samen met diagnosen worden gebruikt. De lijn die het onderzoek heeft gevolgd is van klassieke epidemiologische paradigma's in variatie in tijd, plaats en persoon naar het exploreren van mogelijke nieuwe benaderingen in het onderzoek naar zowel omgevingsfactoren als ook genetische risicofactoren. In de komende tien jaren is het te verwachten dat de manier waarop we onze risicofactoren meten verfijnd zal worden en zullen we ze bestuderen over verschillende hiërarchische/systematische niveaus (maatschappelijk, individueel \& moleculair) in genetisch onderzoek. 


\section{Acknowledgements}

To Jim van Os my promoter- dank je wel. To all my past educational supervisors at The University of Newcastle -Upon-Tyne and of course Robin McCreadie, a big "thank you" for encouraging me to question all our tired assumptions and to look for better ways of doing things using imaginative scientific approaches

A special thanks to all my collaborators, especially my OPCRIT buddies Jane Boydell and Gary Morrison.

To my teachers of epidemiology and statistics Raj Bhopal, Alistair Leyland \& Harper Gilmour I am indebted.

Finally to the patients of Dumfries \& Galloway who participated so enthusiastically in the studies discussed in this thesis and to all the patients I have had the privilege to meet in my professional life it is with deep gratitude that I thank you. 


\section{Curriculum Vitae}

Judith Allardyce is Scottish, went to medical school in Aberdeen before carrying out her psychiatric training in Newcastle-Upon-Tyne, England. She formally training in epidemiology in Glasgow, Scotland and worked as a consultant psychiatrist in South Glasgow for many years and has held academic positions in the Universities of Newcastle \& Glasgow; she has carried out epidemiology studies of severe mental disorder s (especially psychosis) and is now working on a project, examining the joint genetic \& environmental influences for the onset of psychosis, at the University of Maastricht, The Netherlands. 
